\title{
Analysis of the transcriptional repressor function of Arabidopsis glutaredoxin ROXY19
}

\author{
Dissertation \\ for the award of the degree \\ "Doctor of Philosophy" (Ph.D.) \\ Division of Mathematics and Natural Sciences \\ of the Georg-August-Universität Göttingen \\ within the doctoral program Biology \\ of the Georg-August University School of Science (GAUSS) \\ Submitted by \\ Li-Jun Huang \\ from Wuhan, China
}

Göttingen 2016 


\section{Thesis Committee}

Prof. Dr. Christiane Gatz

(Department of Plant Molecular Biology and Physiology)

Prof. Dr. Ivo Feussner

(Department of Plant Biochemistry)

Prof. Dr. Volker Lipka

(Department of Plant Cell Biology)

\section{Members of the Examination Board}

Reviewer: $\quad$ Prof. Dr. Christiane Gatz

(Department of Plant Molecular Biology and Physiology)

Second reviewer: Prof. Dr. Ivo Feussner

(Department of Plant Biochemistry)

Further members of the Examination Board:

Prof. Dr. Volker Lipka

(Department of Plant Cell Biology)

PD Dr. Thomas Teichmann

(Department of Plant Cell Biology)

Prof. Dr. Andrea Polle

(Department of Forest Botany and Tree Physiology)

Jr. Prof. Dr. Cynthia Gleason

(Department of Molecular Plant Science)

Date of the oral examination: 15.02 .2016 
Declaration

Hereby, I declare that this dissertation was undertaken independently and without any unauthorized aid.

I declare that this Ph.D. dissertation has not been presented to any other examining body either in its present or similar form.

Furthermore, I also affirm that I have not applied for a Ph.D. or Dr.rer.nat. at any other higher school of education.

Göttingen,

Li-Jun Huang 


\section{Table of Contents}

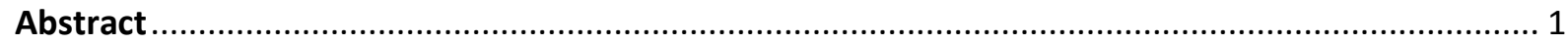

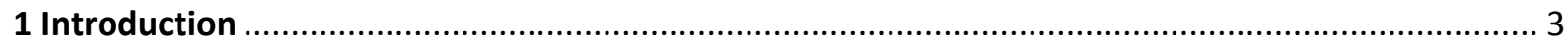

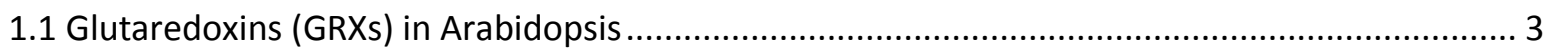

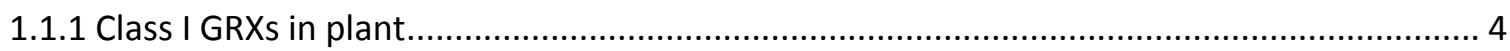

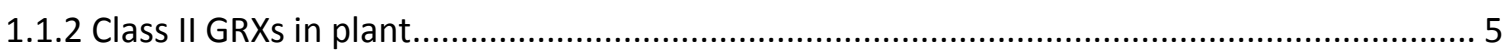

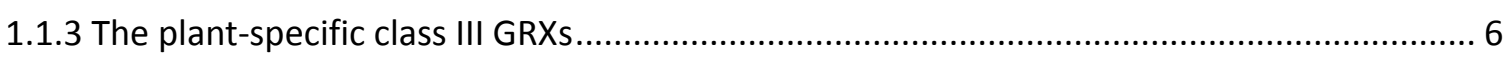

1.2 Class II TGAs play essential roles in stress-related signal transduction pathways ........................ 8

1.3 Possible role of epigenetic modification in SA and ET/JA crosstalk ......................................... 10

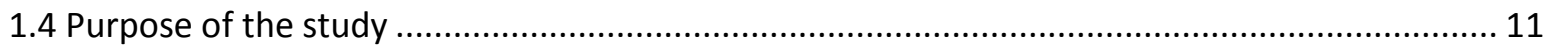

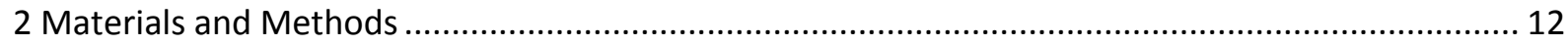

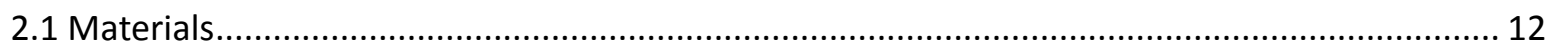

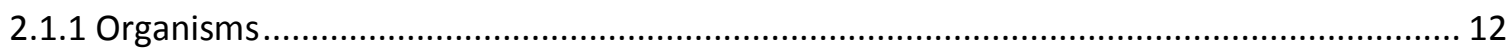

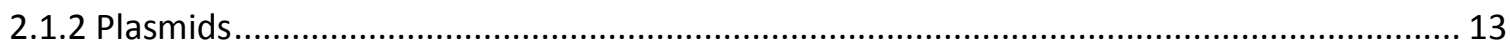

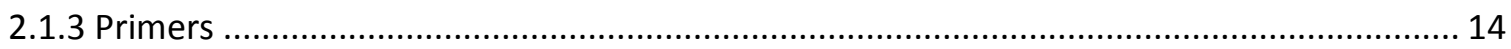

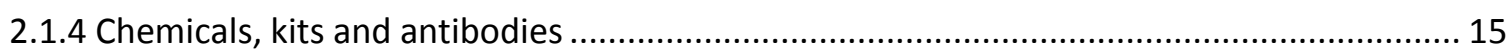

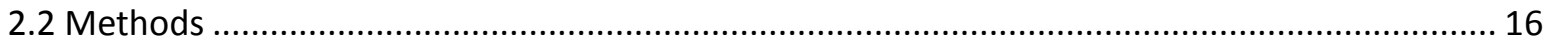

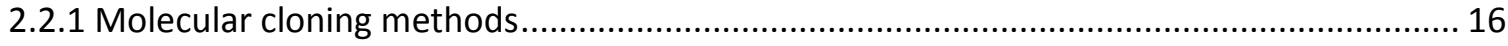

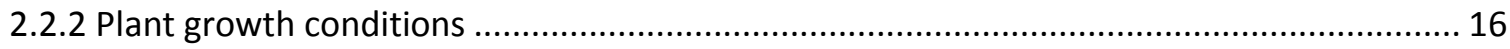

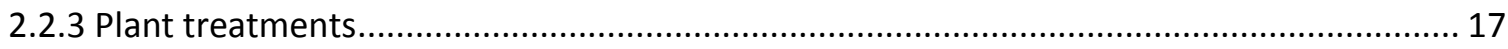

2.2.4 Analysis of gene expression by quantitative real-time PCR …........................................ 18

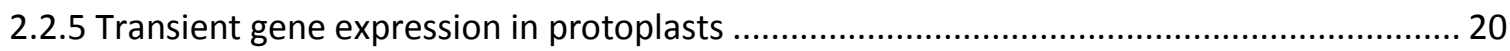

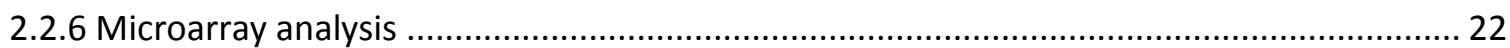

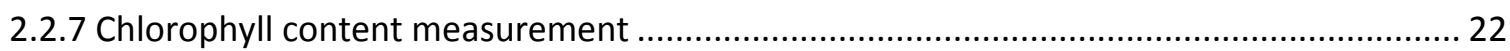

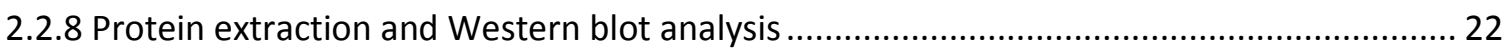

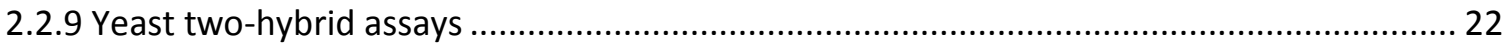

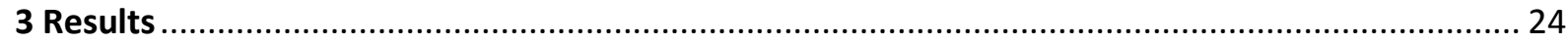

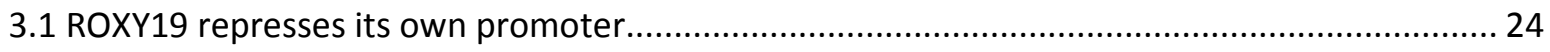

3.1.1 ROXY19 represses its own promoter in transiently transformed protoplasts ..................... 24

3.1.2 ROXY19 requires class II TGA factors to repress the ROXY19 promoter .............................. 25

3.1.3 The ROXY19 active site is not important for the repression activity .................................. 26

3.1.4 ROXY19 may recruit TOPLESS to repress target genes ..................................................... 28

3.1.5 ROXY19 represses JA-induced ROXY19 expression in transgenic plants............................ 29

3.1.6 ROXY19 cannot repress JA-induced ROXY19 expression in the tga256 mutant ................. 29 
3.1.7. The ROXY19 active site is required for repression of its target promoter in plants 29

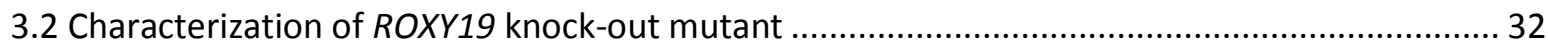

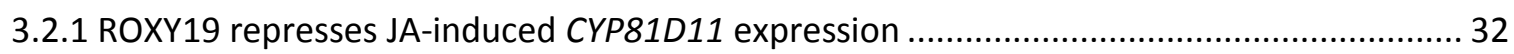

3.2.2 JA-induced CYP81D11 expression is not altered in ROXY19 knock-out mutant .................. 32

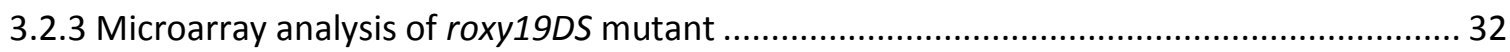

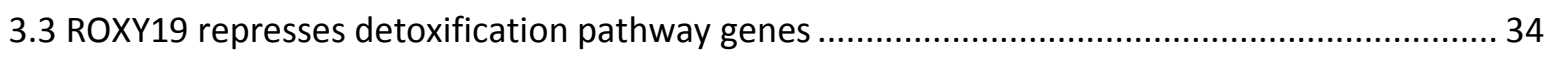

3.3.1 Isolation of ROXY19-regulated genes by microarray analysis ............................................. 34

3.3.2 ROXY19 represses detoxification pathway genes ................................................................ 36

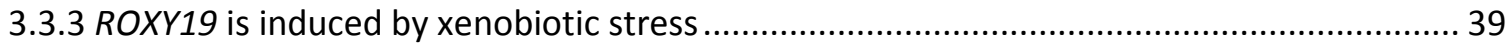

3.3.4 Class II TGA factors are required for TIBA-induced ROXY19 expression .............................. 39

3.4 ROXY19 facilitates susceptibility to necrotrophic fungus Botrytis cinerea …............................ 40

3.5 Development of a chemical inducible ROXY19 expression transgenic line ................................ 40

3.6 Inhibiting DNA methylation cannot recover PDF1.2 expression in ROXY19 transgenic plants... 41

3.7 Characterization of the roles of class II TGA factors in Arabidopsis............................................. 42

3.7.1 The cysteine of class II TGAs is not important for regulating PDF1.2 expression ................. 42

3.7.2 Defense hormone treatment does not influence protein stability of class II TGAs ............. 44

3.7.3 Class I TGAs repress the ET/JA-induced PDF1.2 expression in the absence of class II TGAs 44

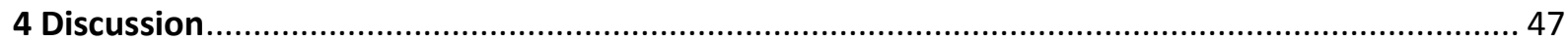

4.1 ROXY19-mediated repression requires a functional active site ................................................ 47

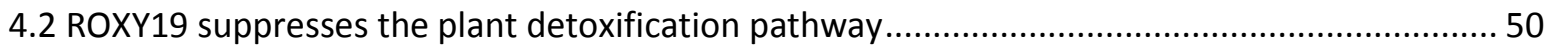

4.3 The repressive effect of ROXY19 is not relieved in roxy19 mutants ........................................ 50

4.4 Role of ROXY19 and TGAs for the crosstalk of SA- and ET/JA-signaling pathway ....................... 50

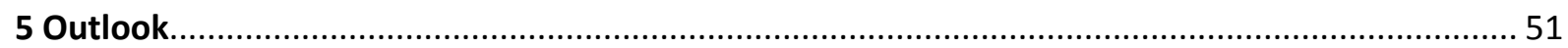

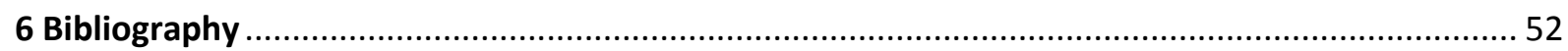

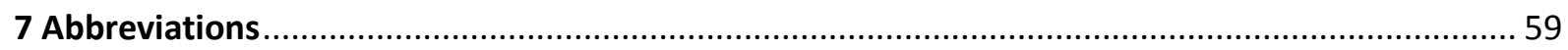

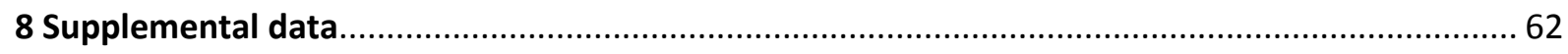

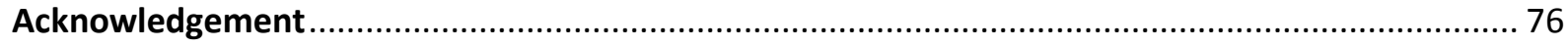

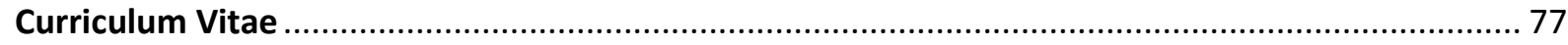




\section{Abstract}

Glutaredoxins (GRXs) are small ubiquitous proteins that are characterized by a thioredoxin (TRX) fold and a glutathione (GSH) reducible active site, which is a CPYC motif in class I GRXs and a CGFS motif in class II GRXs. Biochemically, GRXs can function as thiol-reductases or as scaffold proteins to coordinate Fe-S clusters. Functionally, they are involved in maintaining the reduced state of proteins in the cell and to regulate signaling processes. Only plants encode a third class of GRXs (called ROXYs) which is characterized by a CCMC/S motif. Lossand gain-of-function experiments have so far revealed that ROXYs regulate both developmental and stress-responsive processes.

ROXYs physically and genetically interact with bZIP transcription factors of the TGA family. It has been a long-held hypothesis that ROXYs modulate the activities of corresponding members of the TGA family through redox modification of their cysteine residues. Ectopically expressed ROXY19 suppresses ethylene/jasmonic acid (ET/JA)-induced defense genes through an unknown mechanism that requires the class II TGA transcription factors (namely TGA2, TGA5 and TGA6). The aim of this study was to investigate whether the transcriptional repressor function of ROXY19 involves redox modifications of TGA transcription factors or other targets and to investigate whether its function as a transcriptional repressor can be confirmed by loss of function evidence.

Using the protoplast transient expression assays, we identified that ROXY19 represses expression from its own promoter. The capacity of ROXY19 to repress its own promoter in transiently transformed Arabidopsis protoplasts requires TGA-binding sites in the promoter, TGA factors, the C-terminal ALWL motif and a conserved glycine that is required for glutathione binding. Surprisingly, the conserved active site was not important. Moreover, the single conserved cysteine of class II TGA transcription factors is not important for these proteins to confer activation and ROXY19-repressibility to the promoter. Preliminary data obtained from transient expression assays imply that ROXY19, which interacts with the transcriptional co-repressor TOPLESS (TPL) through the ALWL motif, recruits TPL to repress target gene expression. For reasons yet unknown, the active site is required for the negative effects on endogenous ROXY19 and other target genes when ROXY19 is ectopically expressed in transgenic plants.

Loss of function evidence of the ROXY function might be hampered by potential redundant function of the 21 members in Arabidopsis. Since only ROXY19 is induced by JA and since it can represses the JA-induced TGA-dependent CYP81D11 promoter when ectopically expressed, we hypothesized that CYP81D11 transcription should be hyper-induced in the roxy19 mutant. However, CYP81D11 transcript levels were not influenced by JA-induced ROXY19.

In order to identify potential target genes of ROXY19, the transcriptomes of wild-type, roxy19 and plants ectopically expressing ROXYs were performed. While these experiments 
did not unravel any genes that were affected by the roxy19 allele, genes from all three phases of the detoxification system were found to be down-regulated in plants ectopically expressing ROXY19. This result is consistent with the well-known function of class II TGA factors as activators of the detoxification pathway upon chemical stress. A motif based analysis revealed that the TGA-binding sites are the over-represented motifs in the promoters of ROXY19-repressed genes. Decreased expression of detoxification genes leads to higher sensitivity of the tga256 triple mutant and plants ectopically expressing ROXY19 towards the xenobiotic chemical TIBA (2,3,5-Triiodobenzoic). However, loss of function analysis showed that plants with mutations in roxy19 and roxy18 (ROXY18 is a closest homolog of ROXY19) do not gain enhanced tolerance to TIBA stress. 


\section{Introduction}

\subsection{Glutaredoxins (GRXs) in Arabidopsis}

Glutaredoxins (GRXs) are small ubiquitous proteins which are characterized by the so-called thioredoxin (TRX) fold. This structural motif, which consists of $4 \beta$-sheets and $3 \alpha$-helices ( $\beta 1$ - $\alpha 1-\beta 2-$ $\alpha 2-\beta 3-\beta 4-\alpha 3$ ), is found in TRXs, protein disulfide isomerases (PDIs), glutathione $S$-transferases (GSTs), glutathione peroxidases and GRXs (Lu and Holmgren, 2014). Most of the GRXs use glutathione (GSH) as a cofactor to catalyze the reversible reduction of protein disulfide bridges or protein-GSH mixed disulfide bonds. Other GRXs bind GSH and associate with iron sulfur (Fe-S) clusters. All GRXs contain a conserved active site located on the loop between $\beta 1$ sheet and $\alpha 1$ helix and a GSH binding grove (Lillig et al., 2008). GRXs can reduce substrates by two distinct mechanisms (Fernandes and Holmgren, 2004): the monothiol and the dithiol way. The monothiol mechanism of CxxS-type GRXs uses the cysteine of the active site for a nucleophilic attack on glutathionylated protein, resulting in a GRXGSH-mixed disulfide and the reduced substrate protein. The GRX-GSH-mixed disulfide is further reduced by another GSH molecule, yielding reduced GRX and oxidized glutathione (GSSG); the oxidized glutathione is reduced by the NADPH-dependent glutathione reductase (GR). The dithiol mechanism of CxxC-type GRXs also involves a nucleophilic attack of the first cysteine, but the target is a disulfide and the result of the reaction is a GRX-protein-mixed disulfide. Subsequently, the second cysteine of the active site reduces the GRX-protein-mixed intermediate to release the protein substrate and to form an intramolecular disulfide bond between the two cysteines of the active site. Similar to the monothiol mechanism the oxidized GRX is reduced by GSH. TRXs use a similar dithiol mechanism to reduce target proteins, while different to GRXs, oxidized TRXs are reduced enzymatically by thioredoxin reductases (TRs).

GRXs can also be involved in the assembly of Fe-S clusters in the mitochondrial matrix or in the delivery of Fe-S clusters to client proteins. A fundamental function of Fe-S clusters is to transfer electrons. Other biological roles of Fe-S clusters have been suggested such as sulfur or iron sources and sensor of cellular changes to regulate gene expression. Yeast Grx3 and Grx4 transfer a Fe-S cluster to transcriptional factor Activator of Ferrous Transport 1 (Aft1) which leads to its nuclear export. Under conditions of iron deficiency, insufficient amounts of Fe lead to the depletion of Fe-S clusters causing Atf1 to accumulate in the nucleus and to activate genes compensating the Fe deficiency (Poor et al., 2014). Fe-S clusters are structure combinations of iron and sulfur atoms assembled on scaffold proteins. The most common and simplest cluster - [2Fe-2S] - is constituted by two iron ions bridged by two sulfide ions and coordinated by cysteine of scaffold proteins. For example, structural analysis showed that in the poplar GRXC1 assembled Fe-S cluster, the [2Fe-2S] core unit is coordinated by the first cysteine of active site from two GRXC1 proteins, along with two cysteines from two GSH molecules (Feng et al., 2006 and Figure 1.1).

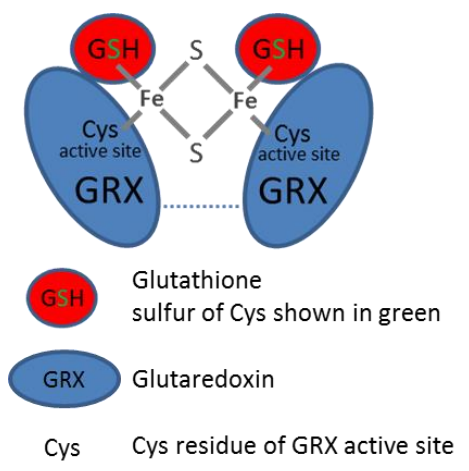

Figure 1.1 A simple proposed model of [2Fe-2S] cluster coordinated by poplar GRXC1. The cluster is composed of a [2Fe2S] core coordinated by two GRX proteins and two GSH molecules. The gray lines indicate chemical bonds between $\mathrm{Fe}$ and sulfur. The dashed line indicates possible interaction between two GRX proteins. Modified from Feng et al. (2006). 
The Arabidopsis genome encodes more than $30 G R X$ and GRX-like genes (Figure 1.2). According to the amino acid of the active site, they are divided into three classes: 1) the CPYC-type (class I) that contains six members: GRXC1, GRXC2, GRXC3, GRXC4, GRXC5 and GRXS12; 2) the CGFS-type (class II) that contains four members: GRXS14, GRXS15, GRXS16 and GRXS17; and 3) the plant-specific CC-type (class III or ROXY) that contains 21 members (Li et al., 2009; Rouhier et al., 2004).

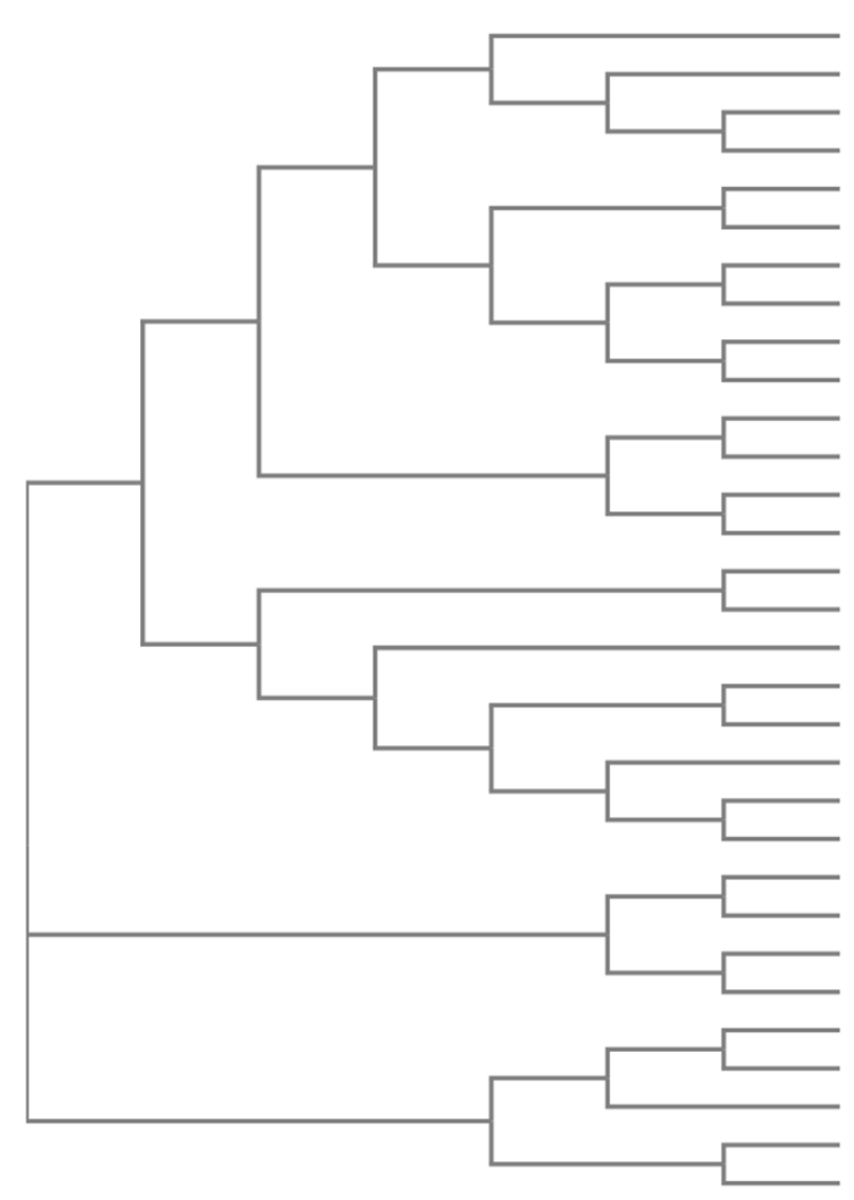

\begin{tabular}{|c|c|c|}
\hline AGI & Name & \\
\hline AT2G38270 & GRXS16 & \\
\hline АT3G15660 & GRXS15/GRX4 & Cloc \\
\hline AT3G54900 & GRXS14/GRXcp & \\
\hline AT4G04950 & GRXS17 & \\
\hline AT1G77370 & GRXC3 & \\
\hline AT5G20500 & GRXC4 & \\
\hline AT2G20270 & GRXS12 & \\
\hline AT4G28730 & GRXC5 & las \\
\hline AT5G40370 & GRXC2/GRX370 & \\
\hline AT5G63030 & GRXC1 & \\
\hline AT1G03850 & ROXY18/GRXS13 & \\
\hline AT1G28480 & ROXY19/GRXC9 & \\
\hline AT4G33040 & ROXY21 & \\
\hline AT5G11930 & ROXY2O & \\
\hline AT1G03020 & ROXY16 & \\
\hline АT3G62930 & ROXY17 & \\
\hline AT5G18600 & ROXY10 & \\
\hline AT4G15690 & ROXY12 & \\
\hline AT4G15700 & ROXY11 & \\
\hline AT4G15680 & ROXY13 & \\
\hline AT4G15660 & ROXY15 & Class III \\
\hline AT4G15670 & ROXY14 & \\
\hline AT2G47880 & ROXY9 & \\
\hline АТ3G62960 & ROXY8 & \\
\hline AT1G06830 & ROXY6 & \\
\hline AT2G30540 & ROXY7 & \\
\hline АТ3G02000 & ROXY1/GRXC7 & \\
\hline AT5G14070 & ROXY2/GRXC8 & \\
\hline АT3G21460 & ROXY3 & \\
\hline AT2G47870 & ROXY5 & \\
\hline AT3G62950 & ROXY4 & \\
\hline
\end{tabular}

Figure 1.2 A phylogenetic tree of the Arabidopsis glutaredoxin family. The phylogenetic tree was derived from the comparisons of protein sequences using the neighbor-joining method in Clustal Omega (EMBL-EBI). Protein sequences of each locus were obtained from TAIR. Gene names were assigned according to Rouhier et al. (2004) or Li et al. (2009).

\subsubsection{Class I GRXs in plant}

The plant CPYC-type GRXs are well characterized in Arabidopsis and poplar. Both GRXC1 and GRXC2 (GRX370) can rescue the yeast grx1 mutant under oxidative stress (Riondet et al., 2012). Genetic studies revealed that single grxc1 and grxc2 mutants showed a decrease in global GRX enzymatic activity as assayed by the reduction of artificial substrates, but no obvious growth phenotype under various environmental stresses. However, a grxc1 grxc2 double mutant is lethal (Riondet et al., 2012). GRXC2 was isolated as an interacting protein of BRASSINOSTEROID INSENSITIVE 1 (BRI1)-ASSOCIATED RECEPTOR-LIKE KINASE 1 (BAK1); in vitro biochemical assays showed that GRXC2 catalyzes BAK1 glutathionylation and inhibits BAK1 peptide kinase activity (Bender et al., 2015). BAK1 is the first target that can be glutathionylated by GRX. 
Concerning the catalytic activities, Sha et al. (1997) assayed the reductase activity of CPYC GRX in vitro using purified protein from rice. The protein exhibited efficient activity in the 2-hydroxyethyl disulfide (HED) reduction assay. Meyer et al. (2007) showed that recombinant GRXC1 is capable of reducing the disulfide bridge of roGFP, an artificial target. roGFP is a redox-sensitive protein, which was engineered from GFP through substitutions of two amino acids by redox-active cysteines (Hanson et al., 2004).

Rouhier et al. (2001) reported the isolation and characterization of the first plant GRX target, a type-C peroxiredoxin (PRX) in poplar. PRXs are thiol-dependent peroxidases that reduce hydrogen peroxide in the presence of an exogenous proton donor. The poplar enzyme was found to use both GRX (poplar GRXC4) and TRX as proton donors (Rouhier et al., 2001). Site-directed mutagenesis analysis found that the first cysteine residue (Cys27) of the active site of GRXC4 is required to promote the catalysis of PRX (Rouhier et al., 2002).

In order to isolate targets of GRX, novel proteomics technologies are being developed. One of these approaches is based on the assumption that an intermediate complex is formed between dithiol GRX (CPYC-type) and its target. Such an intermediate complex has been shown for TRX. A mutation of the last cysteine of the TRX active site stabilizes the complex (Brandes et al., 1993). Thus, expressing an active site mutant of TRX or GRX with a tag allows the trapping and further purification of target proteins. This method has been applied to isolate GRX targets in plants by expressing a polar GRXC4 in Arabidopsis (Rouhier et al., 2005). This led to the identification of 94 putative target proteins, including many peroxiredoxins (PRXs). Serval of these proteins are also known to be targets of TRX. Moreover, it was shown that GRXC4 can reduce and activate peroxide-reducing activity of Arabidopsis type II PRX F (AtPRX IIF) using recombinant protein in vitro (Rouhier et al., 2005).

In addition to being potential oxidoreductases, Rouhier et al. (2007) reported that poplar GRXC1 expressed in E. coli can serve as a scaffold to form Fe-S clusters. The Arabidopsis GRXC5 exists in two forms with different functionalities when expressed in E.coli (Couturier et al., 2011). The monomeric form exhibits deglutathionylation activity, whereas the dimeric form assembles a Fe-S cluster. Sitedirected mutagenesis experiments revealed that the last cysteine of the active site is required for the cluster formation (Couturier et al., 2011). In addition, the Arabidopsis GRXC1, but not its closest homolog GRXC2, was shown to be able to incorporate Fe-S cluster in vitro. However, both GRXC1 and GRXC2 cannot complement the yeast grx5 (class II GRX) mutant defective in Fe-S cluster formation (Riondet et al., 2012).

\subsubsection{Class II GRXs in plant}

Glutaredoxins with a CGFS-type active site were only recently defined as class II GRXs. CGFS-GRXs were initially characterized in yeast ( $\mathrm{Grx} 3, \mathrm{Grx} 4$ and $\mathrm{Grx} 5$ ), and subsequently found in all prokaryotes and eukaryotes. In Arabidopsis, there are four members of this class, GRXS14, GRXS15, GRXS16 and GRXS17.

Cheng et al. (2006) isolated a chloroplast-localized CGFS-type GRXS14 (AtGRXcp) which can rescue the yeast grx5 mutant growth phenotype. Disruption of GRXS14 in planta resulted in oxidative damage of proteins and higher sensitivity to external oxidants (i.e. $\mathrm{H}_{2} \mathrm{O}_{2}$ ). Cheng et al. (2006) also found that GRXS15 (AtGRX4), a close homolog of GRXS14, complements the yeast Grx5 function. In planta, GRXS15 expression is altered under various stresses and is required for resistance against oxidative stress. Bandyopadhyay et al. (2008) showed that - like their poplar orthologs - both 
Arabidopsis GRXS14 and GRXS16 but not GRXS15 can serve as scaffold proteins for the assembly of Fe-S clusters. A most recent publication reported that recombinant GRXS15 can indeed coordinate Fe-S cluster formation. Mutation of GRXS15 in Arabidopsis results in embryonic lethality (Moseler et al., 2015). Cheng et al. (2011) characterized another CGFS-type GRX, GRXS17; they showed that expression of GRXS17 was induced by elevated temperatures and that GRXS17 knockout plants display increased ROS levels and are hypersensitive to high temperature. Consistently, ectopic expression of Arabidopsis GRXS17 in tomato renders the plants more tolerant to heat stress with increased catalase (CAT) enzyme activity and reduced $\mathrm{ROS}\left(\mathrm{H}_{2} \mathrm{O}_{2}\right)$ accumulation (Wu et al., 2012).

Knuesting et al. (2015) found that the shoot apical meristem (SAM) of grxs17 plant was compromised under long-day photoperiod. The authors isolated a GRXS17 interacting partner, the NUCLEAR FACTOR Y SUBUNIT C11/NEGATIVE COFACTOR $2 \alpha$ (NF-YC11/NC2 $\alpha$ ). The $n f-y c 11 / n c 2 \alpha$ mutant plant photocopied the grxs17 mutant phenotype, indicating GRXS17 may play important roles in SAM maintenance by relaying a redox signal to its interaction partner NF-YC11/NC2 $\alpha$. Like GRXS14 and GRXS16, recombinant GRXS17 shows capacity to bind Fe-S clusters and complement the yeast grx5 mutant; however, the authors revealed that GRXS17 has a minor role in Fe-S cluster homeostasis in planta. So far, all members of Arabidopsis class II GRXs are demonstrated to be able to bind Fe-S clusters. Like class II GRXs in other organisms, class II GRXs in plants also play important roles in Fe-S cluster formation.

Class II GRXs are supposed to possess thiol reductase activity as well. Tamarit et al. (2003) showed that yeast Grx5 is not active in the classical HED assay. However, the authors demonstrated that Grx5 is able to reduce disulfides of glutathionylated rat carbonic anhydrase III in a biochemical assay. An active site mutant Grx5 lost the ability to deglutathiolate carbonic anhydrase (Tamarit et al., 2003). Enzymatic targets of class II GRX are missing.

\subsubsection{The plant-specific class III GRXs}

Whereas the CGFS-type and the CPYC-type GRXs are conserved in all eukaryotes, the CC-type is only found in land plants. In contrast to class I and class II GRX, recombinant class III GRX are difficult to obtain. Therefore, data demonstrating their biochemical functions are limited. Only poplar GRXS7.2 was successfully purified from E.coli in the presence of GSH. It displayed typical features of Fe-S cluster (such as brownish color and specific UV/visible light absorption) (Couturier et al., 2010) and poor oxidoreductase activity in the HED assay. Taking an alternative approach, the authors took the SCCMC active site to replace the unusual YCGYC active site of poplar GRXC1. The mutant GRXC1CCMC was indeed able to form Fe-S cluster (Couturier et al., 2010). They also replaced the active site YCPYC of GRXC4 against SCCMC or GCCMS. In contrast to GRXC1, GRXC4 has a strong oxidoreductase activity which was severely reduced in the SCCMC and GCCMS variants. Therefore, it can be tentatively concluded that class III GRXs are potential Fe/S binding proteins with poor oxidoreductase activities, at least with artificial substrates. Another structural hallmark of many class III GRXs is the hydrophobic C-terminal ALWL motif.

Genetic studies on the plant specific CC-type GRXs have revealed interesting results. During flower development, Arabidopsis forms four petals whereas the roxy1 (grxc7) mutant initiates in average only 2.5 petals. At later stages of development, petal morphogenesis is also affected in the roxy1 mutant (Xing et al., 2005). A ROXY1 homolog, ROXY2 (GRXC8) functions redundantly in anther development. A histological analysis of roxy1 roxy2 mutant anthers revealed that sporogenous cell formation fails to occur at anther stage 3 in the mutant. At later stages, pollen mother cells fail to 
differentiate and therefore meiosis is perturbed, resulting in smaller and empty anther locules without pollen grains. Thus, the roxy1 roxy2 double mutant is sterile. Complementation experiments showed that the first but not the last cysteine residue in the active site is crucial for ROXY1 function in petal development (Xing et al., 2005). Interestingly, a conserved glycine residue in the putative GSH-binding site is critical for ROXY1 function (Xing and Zachgo, 2008).

ROXY1 and ROXY2 interact with all 10 members of the TGA transcription factor family (See section 1.2 below), including PERIANTHIA (PAN) (Li et al., 2009; Murmu et al., 2010). Intriguingly, PAN is involved in the determination of flower organ number (Chuang et al., 1999); the pan mutant forms one extra petal. The roxy1 pan double mutant exhibits a similar phenotype as the pan single mutant, indicating that ROXY1 is upstream of $P A N$. Further characterization revealed that nuclear localization and interaction with PAN is required for ROXY1 function. Out of the six cysteine residues present in PAN, Cys340 was shown to be required for rescuing the pan phenotype. Therefore, the hypothesis was put forward that ROXY1 may direct target PAN for post-translational redox modification to inhibit its function.

Murmu et al. (2010) revealed that the tga9 tga10 double mutant, which is deficient in the class IV TGAs, shows a phenotype similar to the roxy1 roxy2 mutant in terms of anther development. The TGA9 and TGA10 expression pattern overlaps with that of ROXY1 and ROXY2 where they positively regulate a common set of genes to promote anther development. Again it is suggested that ROXY1 and ROXY2 influence TGA9 and TGA10 transcriptional activity through redox-modifications.

A maize CC-type GRX, MALE STERILE CONVERTED ANTHER1 (MSCA1), positively regulates shoot meristem size by inhibiting FASCIATED EAR4 (FEA4), an ortholog of the Arabidopsis PAN gene (Pautler et al., 2015; Yang et al., 2015). The msca1 and fea4 have opposite meristem size phenotypes. The double mutant shows a similar increased meristem size like the fea4 single mutant, suggesting that FEA4 is downstream of MSCA1. The last cysteine residue of the active site was found to be required for MSCA1 function in SAM formation (Yang et al., 2015). A direct protein interaction between MSCA1 and FEA4 supports the notion that FEA4 may be redox modified by MSCA1. The maize MSCA1 was reported to control anther development as well, however the corresponding TGA factor is unknown (Chaubal et al., 2003).

The CC-type GRXs may also play roles in plant responses to environmental stresses. The defense hormone salicylic acid (SA) antagonizes the ethylene/jamsonate (ET/JA)-signaling pathway through manipulating transcriptional activity of class II TGAs. Expression of ROXY19 (GRX480 or GRXC9), which was isolated as an interaction partner of TGA2 in a yeast two hybrid screen, is induced by SA in a class II TGA-dependent manner (Ndamukong et al., 2007). ROXY19-mediated repression of JAinduced expression of the marker gene PDF1.2 requires class II TGAs. A direct interaction between ROXY19 and class II TGAs again strongly suggests that SA-induced ROXY19 may inactivate ET/JAinduced transactivation capacity of class II TGA via redox modification. Consistently, the expression of ORA59, a master regulator of the ET/JA pathway and direct target of class II TGA, is repressed in transgenic plant expressing ROXY19. Only ROXYs with a C-terminal ALWL motif repress EIN3activated ORA59 promoter activity in transiently transformed plant protoplasts (Zander et al., 2012). Interestingly, the ALWL motif is also important to complement the roxy1 phenotype indicating that ROXYs exert functions in developmental and defense-associated processes through the same mechanism. Increased susceptibility to necrotrophic pathogen observed in plants ectopically expressing ROXY1 and ROXY19 (Wang et al., 2009; Zander, 2011), may be ascribed to repression of 
the ORA59-mediated defense. However, Wang et al. (2009) suggested that an increased ROS $\left(\mathrm{H}_{2} \mathrm{O}_{2}\right)$ level in ROXY1 overexpressing lines may be a major contribution to the increased susceptibility. Mutation in ROXY18, the closest homolog of ROXY19, resulted in enhanced resistance to the necrotrophic pathogen B. cinerea; unexpectedly, the expression of PDF1.2 was not affected in this mutant (La Camera et al., 2011). The mechanism how ROXY18 facilitate necrotrophic pathogen infection requires further analysis. Laporte et al. (2012) reported that ROXY18 is required for plant protection against oxidative stress. ROXY18 knock-down and overexpression resulted in increased and reduced accumulation of ROS (superoxide radicals), respectively; consistently, the knock-down plants showed reduced tolerance to methyl viologen (MV) and high light $(\mathrm{HL})$ treatments, while the overexpression lines were more resistant.

\subsection{Class II TGAs play essential roles in stress-related signal transduction pathways}

As outlined above, class III GRXs interact with TGA transcription factors (TGAs). TGAs are basic region/leucine zipper motif (bZIP) transcription factors that regulate processes including hormone ( $\mathrm{SA}$ and ET/JA) signaling transduction, xenobiotic detoxification and flower development. The Arabidopsis genome contains ten members of this family that are divided into five classes (Figure 1.3): class I contains TGA1 and TGA4; class II TGA2, TGA5 and TGA6; class III TGA3 and TGA7; class IV TGA9 and TGA10; class $V$ contains only one member PERIANTHIA (PAN). TGA factors bind to variants of the palindrome TGACGTCA, with the half site TGACG being sufficient for binding.

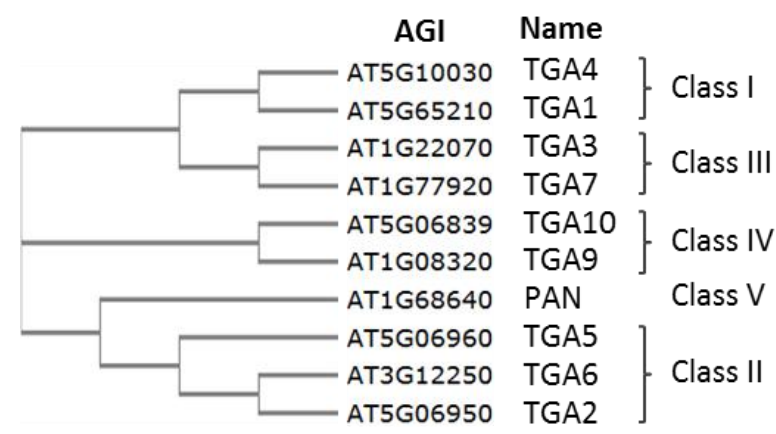

Figure 1.3 A phylogenetic tree of the Arabidopsis TGA family.

Protein sequences were obtained from TAIR and aligned by using Clustal Omega (EMBL-EBI).

This thesis focuses on class II TGA transcription factors TGA2, TGA5 and TGA6 which are positive regulators of the plant defense response "systemic acquired resistance (SAR)". This long lasting and broad range immune response, which is effective against biotrophic and hemi-biotrophic pathogens, is established in distal parts of plants after local infections with biotrophic pathogens. SAR establishment requires the plant phytohormone salicylic acid (SA). The SA-signaling pathway is controlled by the transcriptional coactivator NONEXPRESSER OF PATHOGENESIS-RELATED GENES 1 (NPR1). The NPR1 homologs - NPR3 and NPR4 - were demonstrated to be SA receptors and to regulate NPR1 stability (Fu et al., 2012). Moreover, NPR1 activity is triggered upon an SA-mediated redox-shift which is important for the translocation of NPR1 into the nucleus (Mou et al., 2003) where it interacts with class II TGA transcription factors to induce defense genes (Fan and Dong, 2002). Moreover, cytosol NPR1 was found to be required for SA-triggered antagonistic effect on ET/JA-dependent defense pathway, which is efficient against necrotrophic pathogens (Spoel et al., 2007). 
Class II TGA factors are required for the ET/JA-regulated defense against necrotrophic pathogens (Zander et al., 2010). Increased levels of ET inactivate ER-localized ET receptors, which leads to the inactivation of the kinase CTR. Thus, the substrate of CTR, the ER-localized protein EIN2 becomes dephosphorylated which leads to the cleavage of the protein (Qiao et al., 2012). The soluble domain moves to the nucleus where it leads to stabilization of the transcriptional activator EIN3, which activates other transcriptional activators such as ORA59. Zhu et al. (2011) found that EIN3 is also controlled by the JA signaling pathway since it interacts with JASMONATE ZIM DOMAIN (JAZ) proteins which function as negative regulators of transcription (Chini et al., 2007; Pauwels and Goossens, 2011; Pauwels et al., 2010; Thines et al., 2007). Upon stress, accumulated jasmonoylisoleucine (JA-lle) binds to the F-box protein CORONATINE INSENSITIVE1 (COI1) to facilitate the formation of the COI1-JAZ complex. COI1 mediates the ubiquitination and ultimately degradation of JAZ repressors (Chini et al., 2007; Thines et al., 2007). Thus, the activation of the ORA59 promoter can be achieved by two mechanisms: ET-induced stabilization of EIN3 and JA-induced degradation of the JAZ repressor proteins. Class II TGA proteins bind to the TGACGT element within the ORA59 promoter and strongly enhance ORA59 transcription and transcription of other ET/JA-regulated genes like PDF1.2 under conditions of increased ET levels. However, if JA alone is used to induce the pathway, TGA factors are dispensable (Zander et al., 2010).

Importantly, the SA- and ET/JA-mediated defense responses cannot be activated simultaneously and depending on the timing and intensity of infections with biotrophic and necrotrophic pathogens, one pathway is prioritized over the other (Pieterse et al., 2009). SA suppresses JA-induced expression of PDF1.2 through a mechanism that involves TGA factors and NPR1. In the presence of elevated levels of ET, NPR1 becomes dispensable (Leon-Reyes et al., 2009). Evidence has been provided that SA may manipulate the transcriptional activity of the class II TGA factors at the ORA59 promoter to control the ET/JA-signaling pathway (Zander et al., 2014). In addition, the stability of the ORA59 protein seems to be affected by SA (Van der Does et al., 2013) and the relative contributions of transcriptional and post-transcriptional control mechanisms merging on the ORA59 protein seem to vary depending on the environmental conditions.

Chromatin immunoprecipitation (ChIP) assays unraveled that class II TGAs directly bind to the ORA59 promoter (Zander et al., 2014). A possible mechanism for the inactivation of TGA2 at the ORA59 promoter was postulated after the identification of a plant-specific GRX - ROXY19 - that physically interacts with the class II TGA factors in $\mathrm{Y} 2 \mathrm{H}$ assays. Ectopically expressed ROXY19 strongly represses the ET/JA-induced ORA59 and PDF1.2 expression in a class II TGA-dependent way (Ndamukong et al., 2007). (See section 1.1.3)

Finally, class II TGA factors have been shown to be essential for the regulation of genes involved in the activation of the detoxification pathway that metabolizes xenobiotics to non-toxic forms for longterm storage. In general, plant detoxification uses a three-phase process: transformation (Phase I), conjugation (Phase II) and compartmentation (Phase III) (Sandermann, 1992).

Phase I reaction is the initial step to modify toxic chemicals with reactive substituent groups (i.e. methyl, hydroxyl), which makes the xenobiotics less toxic and more susceptible for Phase II. The major reactions involved in Phase I are oxidation, hydrolysis and reduction. Enzymes catalyzing these reactions are cytochrome P450 (CYP) monooxygenases and hydrolases. In Phase II, metabolites of Phase I are conjugated to endogenous substrates such as sugars, glutathione, and amino acids, resulting in compounds of higher molecular weight and less toxicity. Enzymes involved in Phase II are 
Uridine-diphospho-Glucuronosyltransferases (UGTs) and Glutathione S-Transferases (GSTs). In Phase III, non-toxic products are transported into the vacuole or incorporated into cell wall material. ATPbinding cassette $(A B C)$ transporters and major facilitator superfamily (MFS) transporters are involved in this phase.

Microarray analysis revealed that $56 \%$ (250/446) of the herbicide safener-induced and $60 \%$ (247/411) of the phytoprostane PPA1-induced genes are less expressed in the tga256 mutant, which lacks all three class II TGAs. Further analysis revealed that $60 \%$ and $42 \%$ of these genes contain TGA-binding sites (TGACG motif) in their promoters (Behringer et al., 2011; Mueller et al., 2008). Thus, xenobiotic stresses induce these genes through activating of class II TGA factors. A robust example is the expression of (Fode et al., 2008; Köster et al., 2012). Interestingly, the JA biosynthesis and signaling pathways are also required for xenobiotic-induced CYP81D11 expression. Indeed, several TIBAinduced genes are less well expressed in the JA signaling mutant coil, underpinning the notion that basal JA signaling amplifies the response to chemical stress (Köster et al., 2012). Activation of a subset of the detoxification genes requires the TGA-interacting GRAS protein SCL14 (Fode et al., 2008).

The question is how xenobiotic stresses activate transcriptional activity of class II TGAs. Figure 1.4 shows a list of chemicals that activate gene expression in a class II TGA-dependent manner. Unlike endogenous phytohormones, so far no receptor for specific xenobiotic chemicals has been identified (Ramel et al., 2012). A variety of xenobiotics are revealed to induce a common set of genes (Behringer et al., 2011; Mueller et al., 2008), suggesting a similarity of signaling cascade shared by these chemicals. Various xenobiotic stresses are known to cause ROS accumulation and oxidative stress (Ramel et al., 2012). The as-1-like promoter element is oxidative stress-responsive. ROSinducer (MV) activates the as-1-like promoter element, while antioxidants (DMTU and BHA) prevent SA-induced oxidative damages and inhibit SA-activated as-1-like element (Garretón et al., 2002). Thus a signaling transduction pathway of xenobiotic response might be that xenobiotics induce the accumulation of ROS, which is perceived by class II TGA factors and these TGAs bind to the as-1-like element of xenobiotics responsive gene promoters to activate their expression.
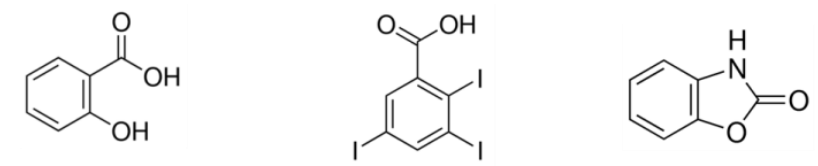

SA (Salicylic acid)

TIBA (2,3,5-Triiodobenzoic acid)

BOA (2-Benzoxazolinone)

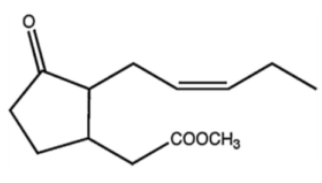

JA (Methyl Jasmonate)

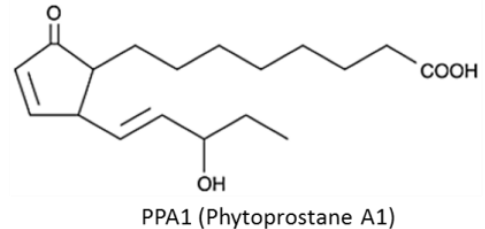

PPA1 (Phytoprostane A1)

Figure 1.4 Chemical structures of SA, JA and some xenobiotics.

\subsection{Possible role of epigenetic modification in SA and ET/JA crosstalk}

The hormone SA influences the expression of approximately $10 \%$ of the Arabidopsis transcriptome. Such a broad effect indicates a possible involvement of chromatin remodeling. Chromatin is a 
dynamic nucleoprotein complex composed of DNA wrapped around histones. Chromatin underlies tight regulation of gene expression by controlling access of transcriptional machinery to DNA. Transcriptional activators and repressors typically recruit enzymes to modify chromatin structure through methylation, acetylation, and phosphorylation of histone tails. Histone acetyltransferases (HAT) and deacetylases (HDA) are responsible to histone acetylation (Kuo and Allis, 1998). In Arabidopsis it has been reported the JA- and ET-inducible HDA6 and HDA19 are involved in regulating the ET/JA-signaling pathway. For instance, HDA6 is recruited via association with the bridging protein JAZ to repress EIN3-mediated transcription of the ERF-branch (Zhu et al., 2011). Conversely, HDA19 is a positive regulator of the ERF-branch. Overexpression of HDA19 confers plant more resistance to necrotrophic pathogen Alternaria brassicicola (Zhou et al., 2005). Thus SA may take control of the ET/JA-signaling through manipulating activities of these enzymes. Using pharmacological treatment and ChIP analysis, Koornneef et al. revealed that histone modification at the PDF1.2 promoter is not altered by SA, indicating chromatin remodeling is not essential for the crosstalk (Koornneef et al., 2008).

\subsection{Purpose of the study}

The aim of this work was to address the function of ROXY19 in Arabidopsis thaliana. Specifically, the question of how ROXY19 represses gene expression was addressed and which types of genes were repressed. Finally, the role of the cysteine residues of class II TGA factors was analyzed. 


\section{Materials and Methods}

\subsection{Materials}

\subsubsection{Organisms}

2.1.1.1 Bacteria

\begin{tabular}{|c|c|c|c|}
\hline Bacteria strain & Description (Genotype) & Usage & Reference \\
\hline Escherichia coli DH5 $\alpha$ & $\begin{array}{l}\mathrm{F}^{-} \text {Ф80lacZ } \Delta \mathrm{M} 15 \Delta(\text { lacZYA-argF) U169 } \\
\text { recA1 endA1 } h s d \mathrm{R} 17\left(\mathrm{r}_{\mathrm{k}}^{-}, \mathrm{m}_{\mathrm{k}}^{+}\right) \text {phoA } \\
\text { supE44 thi-1 gyrA96 relA1 } \lambda^{-}\end{array}$ & $\begin{array}{l}\text { Plasmid } \\
\text { construction }\end{array}$ & $\begin{array}{l}\text { Thermo Fisher } \\
\text { Scientific }\end{array}$ \\
\hline Escherichia coli DB3.1 & $\begin{array}{l}\mathrm{F}-\text { gyrA462 endA1 } \Delta(\mathrm{sr} 1-r e c \mathrm{~A}) \text { mcrB } \\
\text { mrr hsdS20(rB-, mB-) supE44 ara-14 } \\
\text { ga/K2 lacY1 proA2 rpsL20(SmR) xyl-5 } \\
\lambda-\text { leu } m t / 1\end{array}$ & $\begin{array}{l}\text { Plasmid } \\
\text { construction }\end{array}$ & $\begin{array}{l}\text { Thermo Fisher } \\
\text { Scientific }\end{array}$ \\
\hline $\begin{array}{l}\text { Agrobacterium } \\
\text { tumefaciens GV3101 } \\
\text { (pMP90RK) }\end{array}$ & C58; Rif $^{R} ;$ Gent $^{R}$ & $\begin{array}{l}\text { Plant } \\
\text { transformation }\end{array}$ & $\begin{array}{l}\text { Koncz and } \\
\text { Schell, } 1986\end{array}$ \\
\hline
\end{tabular}

\subsubsection{Yeast}

\begin{tabular}{llll}
\hline Yeast strain & Description (Genotype) & Usage & Reference \\
\hline PJ69-4A & MATa trp1-901 leu2-3,112 ura3-52 & Yeast two-hybrid & James et al., \\
& his3-200 gal4D gal80 L LS2::GAL1- & & 1996 \\
& HIS3 GAL2-ADE2 met2::GAL7-lacZ & & \\
\hline
\end{tabular}

\subsubsection{Plants}

\begin{tabular}{lll}
\hline Plant & Description & Reference \\
\hline Col-0 & Arabidopsis thaliana Columbia-0 (Col-0) & TAIR \\
\hline tga256 & $\begin{array}{l}\text { tga2, tga5 and tga6 triple mutant in Col-0 } \\
\text { background }\end{array}$ & Zhang et al., 2003 \\
\hline tga14 & $\begin{array}{l}\text { tga1 and tga4 double mutant in Col-0 } \\
\text { background }\end{array}$ & AG Gatz \\
\hline tga12456 & $\begin{array}{l}\text { tga1, tga2, tga4, tga5 and tga6 pentuple mutant } \\
\text { in Col-0 background }\end{array}$ & AG Gatz \\
\hline tpl-1 & The temperature sensitive dominate topless & Long et al., 2006 \\
& mutant in Landsberg background & \\
\hline ROXY19OE & ROXY19 overexpressing in Col-0 & AG Gatz \\
(35S:HA-ROXY19CCMC) & & AG Gatz \\
\hline ROXY19OE/tga256 & ROXY19 overexpressing in tga256 background & This work \\
\hline 35S:HA-ROXY19CPYC & $\begin{array}{l}\text { Active site mutant (CPYC) ROXY19 overexpression } \\
\text { in Col-0 }\end{array}$ & \\
\hline 35S:HA-ROXY19SSMS & Active site mutant (SSMS) ROXY19 & This work \\
\hline 35S:HA-GRX370 & overexpression in Col-0 & \\
\hline XVE:HA-ROXY19 & G-estradiol-inducible ROXY19 expressing in Col-0 & This work \\
(XVE\#9) & TGA5 overexpressing in Col-0 & This work \\
\hline 35S:TGA5 & &
\end{tabular}




\begin{tabular}{lll}
\hline 35S:TGA5C ${ }^{186} \mathrm{~S}$ & Cys mutant TGA5 overexpression in Col-0 & This work \\
\hline 35S:TGA2 & TGA2 overexpressing in Col-0 & AG Gatz \\
\hline 35S:TGA2C ${ }^{186} \mathrm{~S}$ & Cys mutant TGA2 overexpressing in Col-0 & AG Gatz \\
\hline $\mathrm{pB2} / \mathrm{Col}-0$ & Col-0 transformed with empty vector & This work \\
\hline pB2/tga256 & tga256 mutant transformed with empty vector & This work \\
\hline Nossen & Arabidopsis thaliana Columbia-0 & $\begin{array}{l}\text { NASC (The European } \\
\text { Arabidopsis seeds } \\
\end{array}$ \\
& & stock center) \\
\hline roxy19DS & Arabidopsis Nossen with mutation in ROXY19 & AG Gatz \\
\hline roxy18 roxy19DS & A genetic cross between roxy18 mutant and & AG Gatz \\
& roxy19DS mutant & \\
\hline
\end{tabular}

\subsubsection{Plasmids}

\begin{tabular}{|c|c|c|}
\hline Plasmid & Description & Reference \\
\hline $\begin{array}{l}\text { 35S:GW } \\
\text { (pB2GW) }\end{array}$ & $\begin{array}{l}\text { Destination plasmid for gateway cloning } \\
\text { binary plasmid }\end{array}$ & Karimi et al., 2002 \\
\hline $\begin{array}{l}\text { 35S:HA-GW } \\
\text { (pB2HAGW) }\end{array}$ & $\begin{array}{l}\text { Destination plasmid for gateway cloning } \\
\text { binary plasmid }\end{array}$ & AG Gatz \\
\hline 35S:HA-ROXY19CCMC & Binary plasmid & This work \\
\hline 35S:HA-ROXY19SSMS & Binary plasmid & This work \\
\hline 35S:HA-ROXY19CPYC & Binary plasmid & This work \\
\hline 35S:HA-GRX370 & Binary plasmid & This work \\
\hline 35S:GFP-ROXY19 & Binary plasmid & This work \\
\hline ROXY19:GFP-ROXY19 & Binary plasmid & This work \\
\hline XVE:HA-GW & Destination plasmid gateway cloning & AG Gatz \\
\hline 35S:TGA5 & Binary plasmid & This work \\
\hline 35S:TGA5C ${ }^{186} \mathrm{~S}$ & Binary plasmid & This work \\
\hline XVE:HA-ROXY19 & $\begin{array}{l}\text { Binary plasmid } \\
\beta \text {-estradiol inducible ROXY19 expression }\end{array}$ & This work \\
\hline pUBQ10:HA-GW & $\begin{array}{l}\text { Destination plasmid for gateway cloning } \\
\text { binary plasmid }\end{array}$ & This work \\
\hline pUBQ10:HA & Binary plasmid & This work \\
\hline pUBQ10:HA-ROXY19 & Binary plasmid & This work \\
\hline pUBQ10:HA-ROXY19SCMC & Binary plasmid & This work \\
\hline pUBQ10:HA-ROXY19ACMC & Binary plasmid & This work \\
\hline pUBQ10:HA-ROXY19ACMA & Binary plasmid & This work \\
\hline pUBQ10:HA-ROXY19SSMS & Binary plasmid & This work \\
\hline pUBQ10:HA-ROXY19CPYC & Binary plasmid & This work \\
\hline pUBQ10:HA-ROXY19ALWA & Binary plasmid & This work \\
\hline pUBQ10:HA-ROXY19 $\triangle A L W L$ & Binary plasmid & This work \\
\hline pUBQ10:HA-ROXY18 & Binary plasmid & This work \\
\hline pUBQ10:HA-ROXY18SSLG & Binary plasmid & This work \\
\hline pUBQ10:HA-TPL & Binary plasmid & This work \\
\hline pUBQ10:HA-TPL351 & Binary plasmid & This work \\
\hline pCSV:HA-tpl-1 & Protoplast transient expression & Zhang et al. 2010 \\
\hline pUBQ10:rLuc & $\begin{array}{l}\text { Reference plasmid for protoplast } \\
\text { transient expression }\end{array}$ & AG Gatz \\
\hline ROXY19:Luc & Binary plasmid & This work \\
\hline $\begin{array}{l}\text { mROXY19:Luc } \\
\text { (TGA-binding sites mutant) }\end{array}$ & Binary plasmid & This work \\
\hline
\end{tabular}




\begin{tabular}{lll}
\hline CaMV35S:Luc & Binary plasmid & This work \\
\hline $\begin{array}{l}\text { mCaMV35S:Luc } \\
\text { (as-1-like motif mutant) }\end{array}$ & Binary plasmid & This work \\
\hline pDEST-GBKT7 & Yeast two hybrid plasmid & AG Gatz \\
\hline pDEST-GBKT7-ROXY19 & Yeast two hybrid plasmid & This work \\
\hline pDEST-GBKT7-ROXY19SCMC & Yeast two hybrid plasmid & This work \\
\hline pDEST-GBKT7-ROXY19SSMS & Yeast two hybrid plasmid & This work \\
\hline pDEST-GBKT7-GRX370 & Yeast two hybrid plasmid & This work \\
\hline pDEST-GAD & Yeast two hybrid plasmid & This work \\
\hline pDEST-GAD-TGA2 & Yeast two hybrid plasmid & This work \\
\hline pDEST-GAD-ROXY19 & Yeast two hybrid plasmid & This work \\
\hline pDEST-GAD-ROXY19SCMC & Yeast two hybrid plasmid & This work \\
\hline pDEST-GAD-ROXY19SCMC & Yeast two hybrid plasmid & This work \\
\hline pDEST-GAD- GRX370 & Yeast two hybrid plasmid & This work \\
\hline pDONR207-ROXY19pro & Gateway entry vector & This work \\
\hline pDONR207-PDF1.2pro & Gateway entry vector & This work \\
\hline pDONR207-ROXY19 & Gateway entry vector & This work \\
\hline pDONR207-ROXY19SCMC & Gateway entry vector & This work \\
\hline pDONR207-ROXY19SSMS & Gateway entry vector & This work \\
\hline pDONR207-ROXY19CPYC & Gateway entry vector & This work \\
\hline pDONR207-ROXY19ALWA & Gateway entry vector & This work \\
\hline pDONR207-TPL & Gateway entry vector & This work \\
\hline
\end{tabular}

2.1.3 Primers

\begin{tabular}{|c|c|c|}
\hline Primer & Sequences(5'-3') & Purpose \\
\hline \multicolumn{3}{|l|}{ Sequencing } \\
\hline SEQ-L1 & TCGCGTTAACGCTAGCATGGATCTC & \multirow{2}{*}{ pDONR207 sequencing } \\
\hline SEQ-L2 & GTAACATCAGAGATTTTGAGACAC & \\
\hline pUBQ10seq-fwd & CTAGTTTGTGCGATCGAATTTGTC & pUBQ10 sequencing \\
\hline pB2GW7 fwd & CACAATCCCACTATCCTTCGCA & \multirow{2}{*}{ pB2 sequencing } \\
\hline pB2GW7 rev & CATGAGCGAAACCCTATAAGAACC & \\
\hline pMDC7seq-d1 & GGTAATGCCATGTAATATGCTCG & XVE vector sequencing \\
\hline upperGAD & TTCGATGATGAAGATACCCCACCAAACCC & \multirow{2}{*}{ pDEST-GAD sequencing } \\
\hline lowerGAD & GATGCACAGTTGAAGTGAACTTGCGGGG & \\
\hline pBD2 & TCATCGGAAGAGAGTAGTAAC & \multirow{2}{*}{ pDEST-GBKT sequencing } \\
\hline GBTerm-primer & ATCATAAATCATAAGAAATTCGCCCG & \\
\hline Ocg43Luc & ATGCAGTTGCTCTCCAGCGGTTCC & pBGWL7 sequencing \\
\hline roGFP-NR & AGCTCGACCAGGATGGGCAC & GFP N-terminal reverse \\
\hline \multicolumn{3}{|l|}{ Cloning } \\
\hline \multirow[t]{2}{*}{ ROXY19pro-gw-d1 } & GGGGACAAGTTTGTACAAAAAAGCAGGCTCCGGT & \multirow{4}{*}{ ROXY19 promoter cloning } \\
\hline & GCACAGAAGAATGG & \\
\hline \multirow[t]{2}{*}{ ROXY19pro-gw-r1 } & GGGGACCACTTTGTACAAGAAAGCTGGGTGACAT & \\
\hline & TTTCAAGTATGTTTTTTAAAG & \\
\hline \multirow[t]{2}{*}{ mROXY19pro-d1 } & CATAGCTTCCTGTAGCTCACATCCTTATGGAACCAT & \multirow{4}{*}{$\begin{array}{l}\text { TGA-binding sites mutant } \\
\text { ROXY19 promoter cloning }\end{array}$} \\
\hline & CGTCTAAGCTAGACTTTC & \\
\hline \multirow[t]{2}{*}{ mROXY19pro-r1 } & GAAAGTCTAGCTTAGACGATGGTTCCATAAGGAT & \\
\hline & GTGAGCTACAGGAAGCTATG & \\
\hline PDF1.2pro-gw-d1 & $\begin{array}{l}\text { GGGGACAAGTTTGTACAAAAAAGCAGGCTCC } \\
\text { GCAGCATGCATCGCCGCATC }\end{array}$ & PDF1.2 promoter cloning \\
\hline
\end{tabular}




\begin{tabular}{|c|c|c|}
\hline PDF1.2pro-gw-r1 & \multicolumn{2}{|l|}{ GGGGACCACTTTGTACAAGAAAGCTGGGT } \\
\hline \multirow[t]{2}{*}{ CaMV35Spro-gw-d1 } & GGGGACAAGTTTGTACAAAAAAGCAGGCTCCTGA & \multirow{4}{*}{$\begin{array}{l}\text { CaMV35S promoter } \\
\text { cloning }\end{array}$} \\
\hline & GACTTTTTCAACAAAGG & \\
\hline \multirow[t]{2}{*}{ CaMV35Spro-gw-r1 } & GGGGACCACTTTGTACAAGAAAGCTGGGTGACAT & \\
\hline & TGTTCTCGACTAGAATAGTA & \\
\hline \multirow[t]{2}{*}{ mCaMV35Spro-d1 } & GATATCTCCACTAATATAAGGGACGGTTCACAATC & \multirow{3}{*}{$\begin{array}{l}\text { as-1-like mutant CaMV35S } \\
\text { promoter cloning }\end{array}$} \\
\hline & ССАCT & \\
\hline mCaMV35Spro-r1 & $\begin{array}{l}\text { AGTGGGATTGTGAACCGTCCCTTATATTAGTGGAG } \\
\text { ATATC }\end{array}$ & \\
\hline ROXY19SSMS-d1 & CGGAGAGGATCTTCCATGTCTCATGTGG & \multirow{4}{*}{$\begin{array}{l}\text { Active site mutant ROXY18 } \\
\text { cloning }\end{array}$} \\
\hline ROXY19SSMS-r1 & CCACATGAGACATGGAAGATCCTCTCCG & \\
\hline ROXY18SSLG-d1 & CAAGGAGAGGCTCTTCTTTGGGACACG & \\
\hline ROXY18SSLG-r1 & CGTGTCCCAAAGAAGAGCCTCTCCTTG & \\
\hline \multicolumn{3}{|l|}{ qRT-PCR primer } \\
\hline \multicolumn{3}{|l|}{ QuantiTech (Qiagen) } \\
\hline ANAC032 & Qiagen QT00743561 & \multirow{9}{*}{$\begin{array}{l}\text { Detection of gene } \\
\text { expression }\end{array}$} \\
\hline COR78 & Qiagen QT00840406 & \\
\hline CYP81D11 & Qiagen QT00781662 & \\
\hline JAZ1 & Qiagen QT00861378 & \\
\hline LOX2 & Qiagen QT00785309 & \\
\hline OPR2 & Qiagen QT00894768 & \\
\hline ORA59 & Qiagen QT00852054 & \\
\hline ROXY18 & Qiagen QT00867314 & \\
\hline ROXY19 & Qiagen QT00869715 & \\
\hline \multicolumn{3}{|l|}{ Self-designed primers } \\
\hline PDF1.2-fwd & CTTGTTCTCTTTGCTGCTTTC & \multirow{2}{*}{ PDF1.2 expression } \\
\hline PDF1.2-rev & CATGTTTGGCTCCTTCAAG & \\
\hline PR1-fwd & CTGACTTTCTCCAAACAACTTG & \multirow{2}{*}{ PR1 expression } \\
\hline PR1-rev & GCGAGAAGGCTAACTACAACTAC & \\
\hline ROXY19RT-d2 & TTGGAGGGTTAGATAGGGTTATGG & \multirow{2}{*}{ Endogenous ROXY19 } \\
\hline ROXY19RT-r2 & CGTAAACAACAATTACCAATCAAGATTC & \\
\hline UBQ5-fwd & GACGCTTCATCTCGTCC & \multirow{2}{*}{ UBQ5 expression } \\
\hline UBQ5-rev & GTAAACGTAGGTGAGTCCA & \\
\hline
\end{tabular}

\subsubsection{Chemicals, kits and antibodies}

\subsubsection{Chemicals}

\begin{tabular}{ll}
\hline Chemical & Source \\
\hline 1-Aminocyclopropane-carboxylic acid ( ACC ) & Calbiochem \\
\hline Dimethylsulfoxid (DMSO) & Carl Roth \\
\hline 3-Estradiol ( $\beta$-est) & Sigma-Aldrich \\
\hline 2-Mercaptoethanol & Carl Roth \\
\hline Methyl jasmonate 95\% (MeJA) & Sigma-Aldrich \\
\hline Sodium salicylate & Sigma-Aldrich \\
\hline 2,3,5-Triiodobenzoic acid (TIBA) & Sigma-Aldrich \\
\hline 5-Azacytidine 98\% (5-azaC) & Sigma-Aldrich \\
\hline
\end{tabular}


2.1.4.2 Kits and Enzymes

\begin{tabular}{|c|c|}
\hline Kit and Enzyme & Source \\
\hline Nucleo Spin ${ }^{\circledast}$ Gel and PCR Clean-up & Macherey-Nagel \\
\hline Nucleo Spin ${ }^{\circledast}$ Plasmid & Macherey-Nagel \\
\hline Nucleo Spin ${ }^{\circledast}$ Plasmid PC100 Prep Kit (Midi,Maxi) & Macherey-Nagel \\
\hline Phusion High-Fidelity DNA Polymerase & Thermo Scientific \\
\hline RevertAid Reverse Transcriptase & Thermo Scientific \\
\hline BIOTAQ $^{\text {TM }}$ PCR Kit & Bioline \\
\hline Advantage ${ }^{\circledR} 2$ Polymerase Mix & Clontech \\
\hline Gateway ${ }^{\circledR}$ Technology kit & Invitrogen \\
\hline Pierce 660nm Protein Assay Reagent & Thermo Scientific \\
\hline Ionic Detergent Compatibility Reagent & Thermo Scientific \\
\hline SuperSignal ${ }^{\mathrm{TM}}$ West Femto kit & Thermo Scientific \\
\hline Dual-Luciferase ${ }^{\circledR}$ Reporter Assay System & Promega \\
\hline
\end{tabular}

2.1.4.3 Antibodies

\begin{tabular}{lll}
\hline Antibody & Description & Source \\
\hline anti-HA (ChIP grade) & Monoclonal antibody anti HA tag from rabbit & Abcam \\
\hline anti-TGA2,5 & Polyclonal antiserum anti TGA2,5 from rabbit & AG Gatz \\
\hline anti-rabbit & HRP-conjugated anti rabbit IgG from goat & Life Technologies \\
\hline
\end{tabular}

\subsection{Methods}

\subsubsection{Molecular cloning methods}

Standard molecular cloning was performed according to Molecular Cloning 3rd edition (Sambrook and Russell, 2001). Gateway cloning was performed according to the protocol of Invitrogen (Gateway ${ }^{\circledR}$ Technology User Guide). All plasmids were sequenced by SeqLab (Microsynth). Plasmid maps and sequences were saved electronically as VectorNTI (Invitrogen) files.

\subsubsection{Plant growth conditions}

\subsubsection{Surface sterilization of Arabidopsis seeds}

Arabidopsis seeds were sterilized in a desiccator with a mixture of $100 \mathrm{ml}$ hypochloric solution and 5 $\mathrm{ml}$ hydrochloric acid under fume hood. The desiccator was sealed with a weak vacuum (750 mbar). After $2 \mathrm{~h}$ (for soil grown) or $4 \mathrm{~h}$ (for axenic culture) incubation, the vacuum and the gaseous phase were released under a clean bench.

\subsubsection{Plant growth conditions on soil}

For soil grown plants, surface sterilized seeds were sown on autoclaved soil and stratified in dark at $4^{\circ} \mathrm{C}$ for 2 days. The plants were grown in climate chambers (Johnson Controls) under long day condition ( $16 \mathrm{~h}$ light $/ 8 \mathrm{~h}$ dark, $22^{\circ} \mathrm{C} / 18^{\circ} \mathrm{C}, 80-100 \mu \mathrm{mol} / \mathrm{m}^{2} / \mathrm{s}$ light intensity, $60 \%$ humidity) for hormone spray treatment, or short day condition $\left(8 \mathrm{~h} \mathrm{light} / 16 \mathrm{~h}\right.$ dark, $22^{\circ} \mathrm{C} / 18^{\circ} \mathrm{C}, 80-100 \mu \mathrm{mol} / \mathrm{m}^{2} / \mathrm{s}$ light intensity, $60 \%$ humidity) for pathogen infection. For protoplast isolation, the plants were grown in growth chambers (Percival Scientific) under $12 \mathrm{~h}$ light $/ 12 \mathrm{~h}$ dark, $22^{\circ} \mathrm{C}, 80-100 \mu \mathrm{mol} / \mathrm{m}^{2} / \mathrm{s}$ light intensity. 


\subsubsection{Plant growth conditions on axenic plates}

Surface sterilized seeds were sown on MS-plates under the clean bench and stratified in dark at $4^{\circ} \mathrm{C}$ for 2 days. The plates were placed in climate chambers (Johnson Controls) under $14 \mathrm{~h}$ light $/ 10 \mathrm{~h}$ dark, $22^{\circ} \mathrm{C} / 18^{\circ} \mathrm{C}, 80-100 \mu \mathrm{mol} / \mathrm{m}^{2} / \mathrm{s}, 60 \%$ humidity for 12 to 14 days.

\subsubsection{Plant treatments}

\subsubsection{Arabidopsis transformation}

Arabidopsis plants were transformed by Agrobacterium tumefaciens (strain GV3101) mediated gene transfer using the floral dip method (Clough and Bent, 1998). Agrobacterium were pre-cultured overnight in $5 \mathrm{ml}$ YEB medium supplemented with $20 \mu \mathrm{g} / \mathrm{ml}$ Spectinomycin, $25 \mu \mathrm{g} / \mathrm{ml}$ Gentamycin and $50 \mu \mathrm{g} / \mathrm{ml}$ Rifampicin. This culture was used to inoculate $400 \mathrm{ml} \mathrm{YEB}$ medium supplemented with antibiotics for overnight culture. Agrobacterium cells were harvested by centrifugation at $2000 \mathrm{rpm}$ for $20 \mathrm{~min}$ and the pellet was re-suspended in $500 \mathrm{ml}$ of $5 \%(\mathrm{w} / \mathrm{v})$ sucrose solution. Inflorescences of Arabidopsis plants were dipped into the solution. Dipped plants were kept in dark under high humidity overnight. Positive T1 transformed lines were selected by BASTA (Bayer CropScience) resistance.

\section{YEB medium}

\begin{tabular}{ll}
\hline Ingredient & Amount per 1 I \\
\hline Beef extract & $10 \mathrm{~g}$ \\
Yeast extract & $2 \mathrm{~g}$ \\
Peptone & $5 \mathrm{~g}$ \\
Sucrose & $5 \mathrm{~g}$ \\
Adjust pH to 7.0 & Drops of $1 \mathrm{M} \mathrm{NaOH}$ \\
ddH $_{2} \mathrm{O}$ & to $1 \mathrm{I}$ \\
$1 \mathrm{M} \mathrm{MgSO}_{4}$ (sterile) & add $2 \mathrm{ml}$ after autoclave \\
\hline
\end{tabular}

\subsubsection{Chemical treatment with soil grown Arabidopsis}

For ET and SA treatment with soil grown plants, four-weeks-old plants were sprayed using a bottle diffuser (Carl Roth) with mock $\left(\mathrm{H}_{2} \mathrm{O}\right), 1 \mathrm{mM}$ ACC solution, or solution containing $1 \mathrm{mM}$ ACC and $1 \mathrm{mM}$ $\mathrm{SA}$. The leaves were harvested at $24 \mathrm{~h}$ after treatment and flash frozen in liquid nitrogen.

For JA treatment with soil grown plants, four-weeks-old plants were placed in glass translucent aquarium containing $4.5 \mu \mathrm{M}$ methyl jasmonate deposited on Whatman filter paper. Control plants were incubated under the same conditions without JA. The leaves were harvested at $10 \mathrm{~h}$ after treatment.

For TIBA treatment with soil grown plants, four-weeks-old plants were sprayed using a bottle diffuser (Carl Roth) with mock ( $0.05 \%$ DMSO) or $0.1 \mathrm{mM}$ TIBA solution. TIBA solution was prepared by dilution a $200 \mathrm{mM}$ stock solution in dimethyl sulfoxide (DMSO). The leaves were harvested at $10 \mathrm{~h}$ after treatment.

\subsubsection{Chemical treatment with axenic grown Arabidopsis}

For ET/JA and SA treatment with MS-plates grown plants, Arabidopsis seeds were germinated on MSplates, the plates were placed vertically in climate chambers (Johnson Controls). After 12 days 
growth, Arabidopsis seedlings were transferred onto MS-plates containing: $0.01 \%$ ethanol (mock) or $5 \mu \mathrm{M}$ MeJA in $0.01 \%$ ethanol (JA) and $500 \mu \mathrm{M}$ ACC, or plus $200 \mu \mathrm{M}$ SA as indicated. About 50 seedlings of each treatment were harvested at $48 \mathrm{~h}$ after treatment.

For hormone crosstalk assay in combination with 5-azaC treatment, Arabidopsis seeds were germinated on MS-plates with or without $10 \mu \mathrm{M}$ 5-azaC, the plates were placed vertically in climate chambers (Johnson Controls). After 12 days growth, Arabidopsis seedlings were transferred onto MSplates with or without $10 \mu \mathrm{M} 5$-azaC, and supplemented with: $0.01 \%$ ethanol (mock) or $5 \mu \mathrm{M}$ MeJA in $0.01 \%$ ethanol (JA) and $500 \mu \mathrm{M} \mathrm{ACC}$, or plus $200 \mu \mathrm{M}$ SA as indicated. About 50 seedlings of each treatment were harvested at $48 \mathrm{~h}$ after treatment.

For hormone crosstalk assays with $\beta$-estradiol inducible lines, Arabidopsis seeds were germinated on MS-plates, the plates were placed vertically in climate chambers (Johnson Controls). After 12 days growth, Arabidopsis seedlings were transferred onto MS plates with or without $10 \mu \mathrm{M} \beta$-estradiol and supplemented with: $0.01 \%$ ethanol (mock) or $5 \mu \mathrm{M}$ MeJA in $0.01 \%$ ethanol (JA) and $500 \mu \mathrm{M} \mathrm{ACC}$, or plus $200 \mu \mathrm{M}$ SA as indicated.

For SA growth assay, Arabidopsis seeds were directly germinated on MS-plates with or without 50 $\mu \mathrm{M} S \mathrm{SA}$, the plates were placed horizontally in climate chambers (Johnson Controls). After 12 days growth, the images were taken using Nikon camera.

For TIBA growth assay, Arabidopsis seeds were directly germinated on MS-plates containing $0.025 \%$ DMSO (mock) or $50 \mu \mathrm{M}$ TIBA (dissolved in DMSO as $200 \mathrm{mM}$ stock), the plates were placed horizontally in climate chambers. After 12 days growth, the images were taken using Nikon camera.

For TIBA growth assay with $\beta$-estradiol inducible lines, Arabidopsis seeds were directly germinated on MS-plates containing: $0.05 \%$ DMSO (mock control), $10 \mu \mathrm{M} \beta$-estradiol, $50 \mu \mathrm{M}$ TIBA, or $10 \mu \mathrm{M} \beta$ estradiol plus $50 \mu \mathrm{M}$ TIBA. After 12 days growth, the images were taken using Nikon camera.

\section{Murashige and Skoog (MS) plant medium}

\begin{tabular}{ll}
\hline Ingredient & Amount per $500 \mathrm{ml}$ \\
\hline MS-Medium incl. vitamins (Duchefa) & $2.2 \mathrm{~g}$ \\
MES (Carl Roth) & $5 \mathrm{~g} \mathrm{(1 \% )}$ \\
Adjust pH to 5.7 & Drops of $1 \mathrm{M} \mathrm{KOH}$ \\
dd $\mathrm{H}_{2} \mathrm{O}$ & to $500 \mathrm{ml}$ \\
Agar-Agar, plant (Carl Roth) & $3.4 \mathrm{~g}$ \\
\hline
\end{tabular}

\subsubsection{Inoculation of Arabidopsis with Botrytis cinerea}

Infection of Arabidopsis with B. cinerea was performed as described previously (La Camera et al., 2011). B. cinerea strains BMM was provided by Brigitte Mauch-Mani (University of Neuchatel, Switzerland). Mature leaves of 4-weeks-old Arabidopsis (short day condition) were drop inoculated with $10 \mu \mathrm{l}$ of B. cinerea spore solution $\left(5 \times 10^{4}\right.$ spores $/ \mathrm{ml}$ ) or $1 / 4$ Difco potato dextrose broth (PDB) media as mock control and kept under high humidity. The lesion size was determined with a caliper 4 days after infection. Leaves were harvested and frozen in liquid nitrogen for RNA extraction.

\subsubsection{Analysis of gene expression by quantitative real-time PCR}

\subsubsection{RNA extraction and cDNA synthesis}


Plant tissue harvested was frozen in liquid nitrogen and transferred into $2 \mathrm{ml}$ micro tube (Sarstedt), with a $5.0 \mathrm{~mm}$ stainless steel ball, and homogenized two times with a mixer mill MM301 (Retsch) for $30 \mathrm{sec}$ each at 20 cycles per sec. TRIzol method was used to extract total RNA (Chomczynski, 1993). Fine ground plant tissue ( $200 \mathrm{mg}$ ) was dissolved in $1.3 \mathrm{ml}$ extraction buffer $(380 \mathrm{ml} / \mathrm{l}$ phenol saturated with $0.1 \mathrm{M}$ citrate buffer $\mathrm{pH} 4.3,0.8 \mathrm{M}$ guanidinthiocyanate, $0.4 \mathrm{M}$ ammoniumthiocyanate, $33.4 \mathrm{ml} 3 \mathrm{M} \mathrm{Na}$-acetate $\mathrm{pH}$ 5.2, 5\% glycerol) and shaken at RT for 10 min using Vortex-Genie 2 Mixer (Scientific Industries). Chloroform $(260 \mu \mathrm{l})$ was added to each sample and shaken at RT for additional10 $\mathrm{min}$. The samples were centrifuged at $4^{\circ} \mathrm{C} 12,000 \mathrm{rpm}$ for $30 \mathrm{~min}$. The clear supernatant $(\sim 900 \mu \mathrm{l})$ was transferred into a new $1.5 \mathrm{ml}$ micro tube (Sarstedt) and $325 \mu \mathrm{l}$ of high salt buffer $(1.2 \mathrm{M}$ $\mathrm{NaCl}, 0.8 \mathrm{M} \mathrm{Na}$-citrate) and $325 \mu \mathrm{l}$ of isopropanol was added to each tube. The tubes were inverted and incubated at RT for $10 \mathrm{~min}$. After centrifugation at $4^{\circ} \mathrm{C} 12,000 \mathrm{rpm}$ for $20 \mathrm{~min}$, the supernatant was discarded, the pellets were washed two times with $70 \%$ ethanol. The pellets were allowed to air dry at RT and then dissolved in 20-60 $\mu$ l doubly distilled water.

\section{TRIzol buffer}

\begin{tabular}{ll}
\hline Ingredient & Amount per $500 \mathrm{ml}$ \\
\hline $380 \mathrm{ml} / \mathrm{l}$ phenol with citrate buffer & $190 \mathrm{ml}$ \\
$0.8 \mathrm{M}$ guanidinium thiocyanate & $47.264 \mathrm{~g}$ \\
$0.4 \mathrm{M}$ ammonium thiocyanate & $15.224 \mathrm{~g}$ \\
$33.4 \mathrm{ml} / \mathrm{l} \mathrm{Na}$-acetate (3 M stock) & $16.7 \mathrm{ml}$ \\
$5 \%$ glycerine (100\%) & $25 \mathrm{ml}$ \\
$\mathrm{ddH}_{2} \mathrm{O}$ & to $500 \mathrm{ml}$ \\
\hline Store at $4^{\circ} \mathrm{C}$ & \\
\hline
\end{tabular}

RNA concentration was measured with NanoDrop 2000 spectrophotometer (Thermo Scientific). $1 \mu \mathrm{g}$ total RNA was treated with DNase in a $10 \mu \mathrm{L}$ reaction mixture containing $1 \mu \mathrm{l}$ of 10x DNase I reaction buffer and $1 \mu \mathrm{l}$ DNase I ( $1 \mathrm{U} / \mu \mathrm{l}$, Thermo Scientific). The reaction mixture was incubated at $37^{\circ} \mathrm{C}$ for 30 min followed by addition of $1 \mu \mathrm{l}$ of $25 \mathrm{mM}$ EDTA. The mixture was then incubated at $65^{\circ} \mathrm{C}$ for $10 \mathrm{~min}$ to denatured DNase I. cDNA synthesis was then performed with adding of $0.2 \mu \mathrm{l}$ of $100 \mu \mathrm{M}$ oligo-dT primers and $1 \mu \mathrm{l}$ of $200 \mu \mathrm{M}$ random monomer to the reaction solution. After annealing at $70^{\circ} \mathrm{C}$ for 10 min, $4 \mu \mathrm{l} 5 \times$ RT-buffer, $2 \mu \mathrm{l}$ of $10 \mathrm{mM}$ dNTPs, $0.3 \mu$ l Reverse Transcriptase (RevertAid H Minus Reverse Transcriptase; $200 \mathrm{U} / \mu \mathrm{l}$, Thermo Scientific) and $1.5 \mu \mathrm{l}$ doubly distilled water were added to a final volume of $20 \mu \mathrm{l}$ and the solution was incubated at $42^{\circ} \mathrm{C}$ for $70 \mathrm{~min}$ and afterwards at $70^{\circ} \mathrm{C}$ for $10 \mathrm{~min}$.

\section{Reaction mix and program for cDNA synthesis}

\begin{tabular}{|c|c|c|}
\hline Stock component & Volume & Temperature and duration \\
\hline $1 \mathrm{mg} / \mathrm{ml} \mathrm{RNA}$ & $1 \mu l$ & \multirow{4}{*}{$37^{\circ} \mathrm{C} 30 \mathrm{~min}$} \\
\hline 10x DNase buffer & $1 \mu l$ & \\
\hline $1 \mathrm{U} / \mu \mathrm{l}$ DNase & $1 \mu l$ & \\
\hline $\mathrm{ddH}_{2} \mathrm{O}$ & to $10 \mu \mathrm{l}$ & \\
\hline $25 \mathrm{mM}$ EDTA & $1 \mu l$ & $65^{\circ} \mathrm{C} 10 \mathrm{~min}$ \\
\hline $100 \mu \mathrm{M}$ oligo-dT & $1 \mu l$ & \multirow{2}{*}{$70^{\circ} \mathrm{C} 10 \mathrm{~min}$} \\
\hline $200 \mu \mathrm{M}$ random monomer & $1 \mu l$ & \\
\hline 5x RT-buffer & $4 \mu \mathrm{l}$ & \multirow{3}{*}{$\begin{array}{l}42^{\circ} \mathrm{C} 70 \mathrm{~min} \\
\text { then } 70^{\circ} \mathrm{C} 10 \mathrm{~min}\end{array}$} \\
\hline $10 \mathrm{mM}$ dNTPs & $2 \mu l$ & \\
\hline $200 \mathrm{U} / \mu \mathrm{l}$ Reverse Transcriptase & $\begin{array}{l}0.2 \mu l \\
\text { to } 20 \mu l\end{array}$ & \\
\hline
\end{tabular}




\subsubsection{Quantitative real-time PCR (qRT-PCR)}

For quantification of CDNA, qRT-PCR was performed with Ubiquitin 5 (UBQ5) as reference gene and the fluorescence intensity was measured with the MyiQ ${ }^{\text {TM }}$ PCR Detection System (BioRad). The amplification mix consisted of $1 \mu \mathrm{l}$ of 1:10 diluted CDNA, 1x NH4-reaction buffer (Bioline), $2 \mathrm{mM}$ $\mathrm{MgCl} 2,100 \mu \mathrm{M}$ dNTPs, $0.4 \mu \mathrm{M}$ primers, $0.25 \mathrm{U}$ BIOTaq DNA polymerase, $10 \mathrm{nM}$ fluoresceine (BioRad), 100,000x diluted SYBR Green I (Cambrex) solution and $17.2 \mu \mathrm{l}$ doubly distilled water (final volume 25 $\mu$ ). PCR started with a denaturation for $6 \mathrm{~min}$ and $95^{\circ} \mathrm{C}$ followed by 40 cycles of $20 \mathrm{~s}$ at $95^{\circ} \mathrm{C}, 20 \mathrm{~s}$ at $55^{\circ} \mathrm{C}$ and $40 \mathrm{~s}$ at $72^{\circ} \mathrm{C}$. Calculation of relative gene expression was done with the $2^{\text {-CT(gene of interest)- }}$ ${ }^{\mathrm{CT}(\text { reference gene)] }}$ method (Schmittgen and Livak, 2008).

\section{Reaction mix for qRT-PCR using BIOTAQ DNA Polymerase}

\begin{tabular}{|c|c|}
\hline Stock component & Volume in a $25 \mu$ l reaction \\
\hline $10 \mathrm{X} \mathrm{NH}_{4}$ reaction buffer & $2.5 \mu l$ \\
\hline $\mathrm{MgCl}_{2} 50 \mathrm{mM}$ & $1 \mu l$ \\
\hline dNTPs 40 mM (10 mM each) & $0.25 \mu \mathrm{l}$ \\
\hline $\mathrm{F}$ and $\mathrm{R}$ primers (each $4 \mathrm{mM}$ ) & $2.5 \mu l$ \\
\hline Sybr Green (1/1000) & $0.25 \mu \mathrm{l}$ \\
\hline Fluorescein (1 mM) & $0.25 \mu l$ \\
\hline BIOTAQ DNA Polymerase (2500 U) & $0.05 \mu \mathrm{l}$ \\
\hline cDNA template $(\sim 0.05 \mu \mathrm{g})$ & $1 \mu l$ \\
\hline
\end{tabular}

Program of qRT-PCR cycler using BIOTAQ DNA Polymerase

\begin{tabular}{lll}
\hline Cycle step and repeat & Temperature and duration & Cycles \\
\hline Initial denaturation & $95^{\circ} \mathrm{C}, 90 \mathrm{sec}$ & 1 \\
\hline Denaturation & $95^{\circ} \mathrm{C}, 20 \mathrm{sec}$ & \\
Annealing & $55^{\circ} \mathrm{C}, 20 \mathrm{sec}$ & 39 \\
Extension & $72^{\circ} \mathrm{C}, 40 \mathrm{sec}$ & \\
\hline Final extension & $72^{\circ} \mathrm{C}, 4 \mathrm{~min}$ & 1 \\
\hline \multirow{3}{*}{ Generation of melt curve } & $95^{\circ} \mathrm{C}, 1 \mathrm{~min}$ & 1 \\
& $55^{\circ} \mathrm{C}, 1 \mathrm{~min}$ & 1 \\
& $55^{\circ} \mathrm{C}, 10 \mathrm{sec}\left(+0.5^{\circ} \mathrm{C} / \mathrm{cycle}\right)$ & 81 \\
\hline
\end{tabular}

\subsubsection{Transient gene expression in protoplasts}

\subsubsection{Arabidopsis protoplasts isolation}

Protoplasts isolation was performed according to the method described by Sheen laboratory (Yoo et al., 2007). The lower surface of leaves of 4-6 week old plants grown in 12/12 light condition was lightly scratched with a razor blade and placed in a petri dish containing $10 \mathrm{ml}$ enzyme solution. After incubation overnight in 12/12 light condition the digested solution was filtrated (75 $\mu \mathrm{m}$ mesh) and the protoplasts were centrifuged ( $2 \mathrm{~min}, 780 \mathrm{rpm}$, soft start and stop). The pellet was washed two times with $10 \mathrm{ml}$ W5 solution (1 min, $780 \mathrm{rpm}$, soft start and stop) and afterwards the protoplasts were re-suspended in W5 solution and incubated on ice before transfection.

\section{Enzyme solution}

\begin{tabular}{ll}
\hline Ingredient & Amount per $50 \mathrm{ml}$ \\
\hline Cellulase & $0.625 \mathrm{~g}$ \\
Maceroenzyme & $0.150 \mathrm{~g}$
\end{tabular}




\begin{tabular}{ll}
$0.75 \mathrm{M} \mathrm{Mannitol}$ & $26.6 \mathrm{ml}$ \\
$0.5 \mathrm{M} \mathrm{KCL}$ & $2 \mathrm{ml}$ \\
$0.5 \mathrm{M} \mathrm{MES}$ & $2 \mathrm{ml}$ \\
$1 \mathrm{M} \mathrm{CaCl}_{2}$ & $5 \mathrm{ml}$ \\
$\mathrm{ddH}_{2} \mathrm{O}$ & to $50 \mathrm{ml}$ \\
\hline \multicolumn{2}{l}{ Filter sterile and store at $4^{\circ} \mathrm{C}$} \\
\hline
\end{tabular}

\subsubsection{PEG-mediated plasmid transfection into protoplasts}

For PEG-mediated transfection of the protoplasts, the W5 solution covering the protoplasts was removed carefully and the pellet was re-suspended in MMG solution. Protoplasts in MMG solution (200 $\mu \mathrm{l}$ per transfection) were transferred into a $2 \mathrm{ml}$ Eppendorf tube containing $220 \mu \mathrm{l} 40 \%$ PEG4000 solution and $20 \mu \mathrm{l}$ plasmid DNA mix $(7.5 \mu \mathrm{g}$ effector plasmid, $5.0 \mu \mathrm{g}$ reporter plasmid and 1.0 $\mu \mathrm{g}$ reference plasmid). The solution was gently mixed and incubated at RT for $30 \mathrm{~min}$. Then $800 \mu \mathrm{l}$ W5 buffer was added and gently mixed by inverting the tube. The supernatant was removed after centrifugation at $780 \mathrm{rpm}$ for $2 \mathrm{~min}$ and protoplasts. The supernatant was removed and the pellet was re-suspended in $300 \mu \mathrm{WI}$ solution, mixed gently and incubated overnight in 12/12 light condition.

W5 buffer

\begin{tabular}{ll}
\hline Ingredient & Amount per $50 \mathrm{ml}$ \\
\hline $1 \mathrm{M} \mathrm{NaCl}$ & $7.7 \mathrm{ml}$ \\
$1 \mathrm{M} \mathrm{CaCl}_{2}$ & $6.25 \mathrm{ml}$ \\
$0.5 \mathrm{M} \mathrm{KCl}$ & $0.5 \mathrm{ml}$ \\
$0.5 \mathrm{M} \mathrm{MES}$ & $0.2 \mathrm{ml}$ \\
$\mathrm{ddH_{2 }} O$ & to $50 \mathrm{ml}$ \\
\hline Filter sterile and store at $4^{\circ} \mathrm{C}$ \\
\hline
\end{tabular}

MMG buffer

\begin{tabular}{ll}
\hline Ingredient & Amount per $50 \mathrm{ml}$ \\
\hline $0.75 \mathrm{M} \mathrm{Mannitol}$ & $26.6 \mathrm{ml}$ \\
$0.5 \mathrm{M} \mathrm{MgCl}_{2}$ & $1.5 \mathrm{ml}$ \\
$0.5 \mathrm{M} \mathrm{MES}$ & $0.4 \mathrm{ml}$ \\
$\mathrm{dd}_{2} \mathrm{O}$ & to $50 \mathrm{ml}$ \\
\hline
\end{tabular}

Filter sterile and store at $4^{\circ} \mathrm{C}$

40\% PEG 4000 solution

\begin{tabular}{ll}
\hline Ingredient & Amount per $50 \mathrm{ml}$ \\
\hline $\mathrm{PEG} 4000$ & $20 \mathrm{~g}$ \\
$0.75 \mathrm{M} \mathrm{Mannitol}_{1 \mathrm{M} \mathrm{CaCl}}$ & $13.3 \mathrm{ml}$ \\
$\mathrm{ddH_{2 } \mathrm { O }}$ & $5 \mathrm{ml}$ \\
\hline Filter sterile and store at $4^{\circ} \mathrm{C}$ \\
\hline
\end{tabular}

\subsubsection{Dual luciferase assay}

Luciferase activities of transfected protoplasts were determined with the Dual Luciferase Assay Kit (Promega) using the CentroXS ${ }^{3}$ LB 960 luminometer (Berthold Technologies). After removing the WI solution, protoplasts were frozen in liquid nitrogen. The frozen protoplasts were dissolved in $20 \mu \mathrm{l}$ 
PassivLysisBuffer and kept on ice. Then $3 \mu$ of the each lysate was transferred into a single well of a 348 well-plate, each well was measured as followed: $30 \mathrm{sec}$ waiting time, injection of $15 \mu$ L LARII, 5 sec waiting time, measurement of fLuc activity for $5 \mathrm{sec}$, injection of $15 \mu$ Stop\&Glo, measurement of rLuc activity for $5 \mathrm{sec}$.

\subsubsection{Microarray analysis}

For microarray analysis, Arabidopsis seeds were germinated on MS-plates and grown vertically in climate chamber (Johnson Controls). After two weeks, approximately 50 seedlings were harvested as one pool. The experiments were repeated four times. RNA was extracted using TRIzol method. RNA samples were sent to the Centre for Organismal Studies (COS) at Heidelberg where the microarray analysis was performed with Arabidopsis GeneChip ${ }^{\circledR}$ Gene 1.0 ST Arrays (Affymetrix). Up- or downregulated genes between different lines were determined by fold change more than two-fold and $p$ value less than 0.05. For cis-element enrichment analysis, the Motif Mapper (Berendzen et al., 2012) was deployed to define significant distribution alterations compared with 1000 randomly composed, equally sized reference promoter datasets; $1000 \mathrm{bp}$ upstream regions of Arabidopsis genes were downloaded from TAIR. Genes down-regulated in 35S:HA-ROXY19CCMC\#8 were subjected to AgriGO database to investigate the gene ontology (Du et al., 2010).

\subsubsection{Chlorophyll content measurement}

Approximately 25 two-weeks-old seedlings from MS-plates were collected as pool. Fresh weight was measured before the seedlings were homogenized in liquid nitrogen. Total chlorophyll was extracted with $80 \%$ acetone $(\mathrm{v} / \mathrm{v})$ for $24 \mathrm{~h}$ in darkness. After a centrifuge at $13000 \mathrm{rpm}$ for $2 \mathrm{~min}$, absorbance of the supernatant was measured at 645 and $663 \mathrm{~nm}$ (Biochrom Libra S11). Total chlorophyll content was calculated using $\left(20.2 \times A_{645}+8.02 \times A_{663}\right) / \mathrm{g}$ fresh weight.

\subsubsection{Protein extraction and Western blot analysis}

Proteins were extracted from homogenized plant tissue under denaturing conditions. The deep frozen plant powder ( $200 \mu \mathrm{l}$ ) was thaw in $600 \mu \mathrm{l}$ extraction buffer (4 M urea, 16.6\% glycerol, 5\% SDS, $0.5 \% \beta$-mercaptoethanol) with shaking at $65^{\circ} \mathrm{C}$ for $10 \mathrm{~min}$. Afterwards the solution was centrifuged for $20 \mathrm{~min}$ at $13000 \mathrm{rpm}$ at RT and the supernatant was used for SDS-PAGE and Western blot analysis. Protein concentration was determined using the Pierce 660nm Protein Assay kit (Thermo Scientific).

\section{Protein extraction buffer}

\begin{tabular}{ll}
\hline Ingredient & Final concentration \\
\hline Urea & $4 \mathrm{M}$ \\
Glycerol & $16.6 \%(\mathrm{v} / \mathrm{v})$ \\
SDS & $5 \%(\mathrm{w} / \mathrm{v})$ \\
$\beta$-mercaptoethanol & $0.5 \%(\mathrm{w} / \mathrm{v})$ \\
\hline
\end{tabular}

\subsubsection{Yeast two-hybrid assays}

A high efficiency transformation protocol was used to transfer PJ69-4a yeast strain in yeast-twohybrid assays. The yeast cells were grown overnight in $20 \mathrm{ml} \mathrm{YPAD} \mathrm{medium} \mathrm{at} 29^{\circ} \mathrm{C}$ on a shaker $(200$ rpm). Overnight culture was sub-cultured into new YPAD media and incubated at $29^{\circ} \mathrm{C}$ until the OD600 was between 0.6-1.2. Yeast cells were collected and wash with $\mathrm{ddH}_{2} \mathrm{O}$ by centrifugation at $4000 \mathrm{rpm}$ for $5 \mathrm{~min}$ at room temperature in $50 \mathrm{ml}$ falcon tube. The cells were re-suspended in $1 \mathrm{ml}$ of water and transferred into a sterile Eppendorf tube before briefly centrifuging at $13,000 \mathrm{rpm}$ to 
pellet the cells. Cells were re-suspended in $1 \mathrm{ml}$ of $100 \mathrm{mM} \mathrm{LiAc} \mathrm{pH} 7.5$ and were distributed as 100 $\mu \mathrm{l}$ aliquots into $1.5 \mathrm{ml}$ centrifuge tube (number of aliquots depend on number of transformation reactions). Supernatant was removed by brief centrifugation followed by adding a transformation

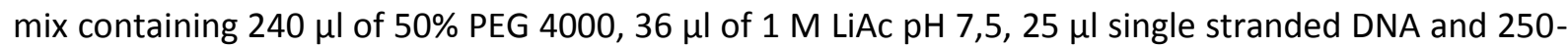
$500 \mathrm{ng}$ of plasmid. The mixture was vortexed vigorously to re-suspend the cells. Next, the mixture was incubated at $30^{\circ} \mathrm{C}$ for 25 min with occasional shaking. Transformation was performed by heat shock. The yeast was incubated at $42^{\circ} \mathrm{C}$ for $25 \mathrm{~min}$. Subsequently, cells were centrifuged at $4000 \mathrm{rpm}$ for $10 \mathrm{sec}$ and supernatant was removed. Yeast cells were re-suspended in $200 \mu \mathrm{l}$ of sterile water. Aliquots were spread onto suitable selective drop out media. Plates were allowed to air dry and incubated at $29^{\circ} \mathrm{C}$ for 3 days or until the colonies developed. 


\section{Results}

\subsection{ROXY19 represses its own promoter}

\subsubsection{ROXY19 represses its own promoter in transiently transformed protoplasts}

Previously, Zander et al. have shown that ectopically expressed ROXY19 represses ET/JA-induced ORA59 expression (Zander et al., 2012). Since ORA59 activates the PDF1.2 promoter, PDF1.2 is also not activated in 35S:ROXY19 plants. Importantly, repression of PDF1.2 promoter activity depends on class II TGA transcription factors. Likewise, ROXY19 represses EIN3-activated ORA59 promoter activity in transiently transformed protoplasts. However, ROXY19-mediated repression only partially depends on class II TGA factors in this assay (Zander et al., 2012). We were interested in testing whether ROXY19 represses its target promoters through redox modification of class II TGA factors. In order to allow structure function analysis of class II TGA factors in protoplasts, we set out to identify a promoter which is repressed by ROXY19 in a class II TGA-dependent manner in transiently transformed protoplasts.

The Cauliflower Mosaic Virus 35S (CaMV35S) promoter and the promoters of the ROXY19 and GSTF8 genes contain as-1-like elements, which are characterized by variants of two TGA-binding TGACGTCA palindromes spaced by $4 \mathrm{bp}$ (Figure 3.1A). Using the transient expression assay in Arabidopsis mesophyll protoplasts, we sought to evaluate whether these promoters are repressed by ROXY19.

For construction of reporter vectors, the promoter region including the $5^{\prime}$-UTR from position - 1480 to +1 relative to the annotated translational start site (ATG) of ROXY19 (AT1G28480) and the promoter region from position -1070 to the ATG of GSTF8 (AT1G28480) were cloned from the Arabidopsis Col-0 genome. The viral CaMV35S promoter was cloned using the pB2GW7-HA plasmid (Weiste et al., 2007) as template. The CaMV35S promoter region spans from position - 442 to the ATG site of HA tag.

Binary vectors including reporter vector which express firefly lucifease (fLuc) under the control of target promoters (ROXY19, GSTF8 or CaMV35S) and an effector vector which expresses ROXY19 under the control of the Arabidopsis UBQ10 promoter were transformed into protoplasts. In order to normalize the transformation efficiency, a reference vector which expresses Renilla luciferase ( $r$ Luc) controlled by the UBQ10 promoter was used (Figure 3.1B). Importantly, the UBQ10 promoter is not affected by ROXY19 (Figure 3.1G). Dual Luc activity tests show that promoter activities of ROXY19 and CaMV35S are significantly (more than six-fold) repressed in the presence of ROXY19 (Figure 3.1C and 3.1D). Expression from the GSTF8 promoter was very low and only slightly repressed (Figure 3.1E). Further mutagenesis analysis showed that the CaMV35S promoter activity is abolished when the as-1-like element is mutated (Figure 3.1D). Mutation of the as-1-like element and another TGACG motif (at position -70 to -66) strongly compromised the ROXY19 promoter activity (Figure $3.1 \mathrm{C}$ ).

It had been reported that the C-terminal ALWL motif is important for the function of ROXYs when regulating petal number or when repressing ORA59 promoter activity (Li et al., 2009; Zander et al., 2012). To address whether the motif is required for repression of its own promoter, we constructed ROXY19 mutants where the ALWL motif was deleted (ROXY19 $A$ ALWL) or the last leucine was replaced by an alanine (ROXY19ALWA). Indeed, both ROXY19 mutants lost their repression activities on the ROXY19 promoter (Figure 3.1F), indicating that the repression requires the C-terminal ALWL motif. 


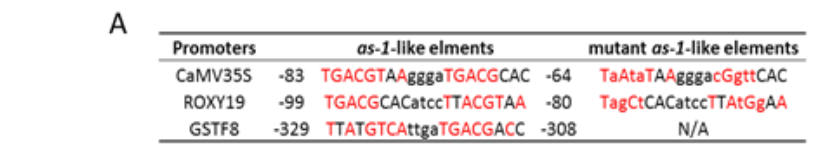

B
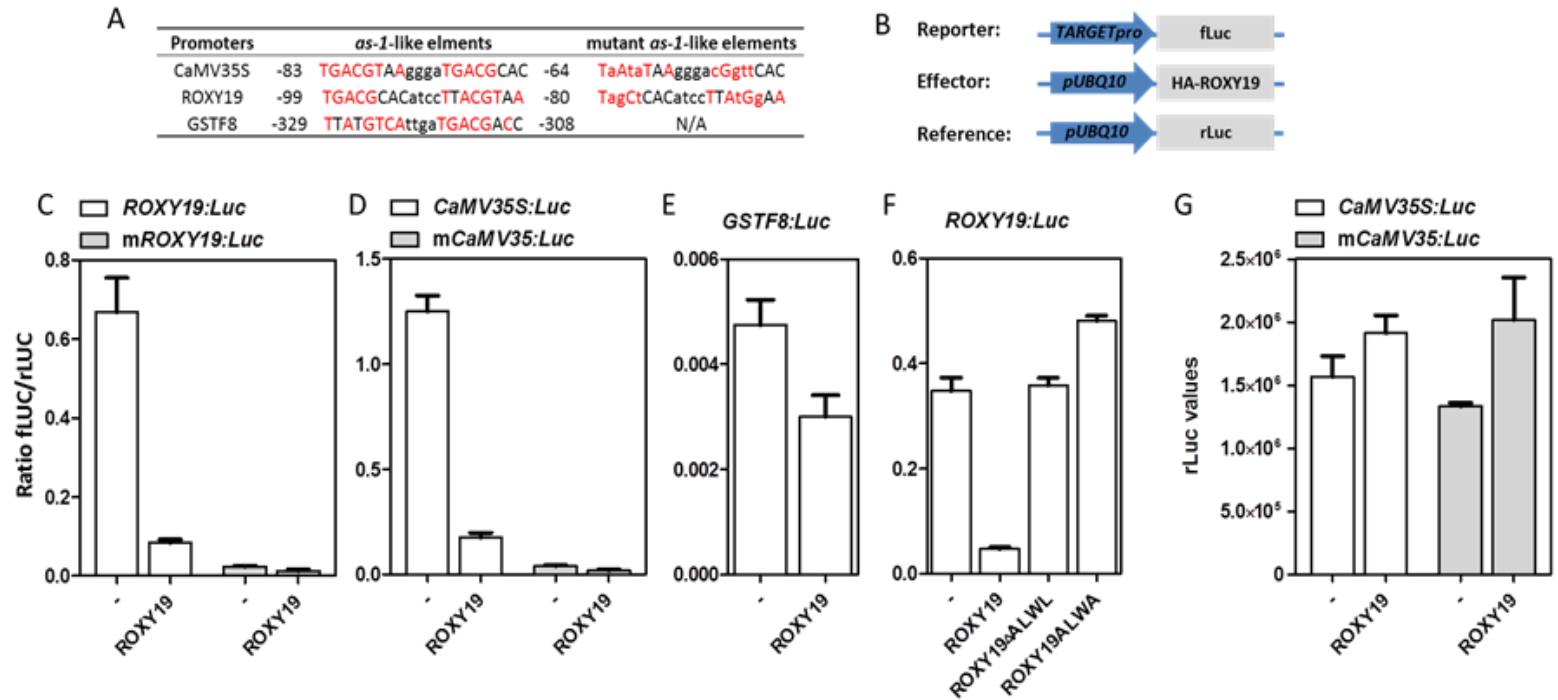

Figure 3.1 ROXY19 specifically represses as-1-containing promoters in transiently transformed Arabidopsis protoplasts. (A) Sequences of as-1-like elements of promoters used to test ROXY19 repression activity. The numbers indicate their positions relative to the translational start sites $(+1)$. Conserved nucleotides within the two 8-bp palindromes (uppercase letters) are highlighted in red. The sequences of the respective mutated as-1-like elements are shown next to the wild-type sequences. For ROXY19 promoter mutagenesis analysis, another mutation of a putative TGA-binding site (from TGACG to ctAaG at position -70 to -66) is not shown here. Point mutations are shown in in lowercase letters. (B) Schematic representation of the reporter, effector and reference constructs. Sequence of target promoters are placed upstream of the firefly luciferase gene ( $L L C$ ). The ROXY19 coding region is fused to the HA-tag and placed downstream of the UBQ10 promoter. The renilla luciferase gene $(r L u c)$ is placed downstream of the UBQ10 promoter. (C) - (E) Transient expression assays. Expression of the target promoters (CaMV35S, ROXY19 and GSTF8) or corresponding mutant variants fused to the fLuc gene was analyzed in transiently transformed Arabidopsis mesophyll protoplasts in the absence or presence of ROXY19 effector. An empty plasmid was used when ROXY19 effect plasmid was absent (-). Luc activities were determined $16 \mathrm{~h}$ after transfection. Values are means of four replicates $( \pm \mathrm{SE})$. (F) Transient expression assays with ROXY19 promoter and ROXY19 effectors either lacking the C-terminal ALWL motif (ROXY19 $A$ ALWL) or containing a mutant ALWL motif (ROXY19ALWA). (G) Arbitrary luminescence units of rLuc in Figure 1D are shown.

\subsubsection{ROXY19 requires class II TGA factors to repress the ROXY19 promoter}

Taking the advantage of Arabidopsis protoplast transient gene expression assay, we analyzed the role of class II TGA factors in ROXY19-mediated repression of the ROXY19 promoter. First, we compared ROXY19 repression activities in protoplasts derived from Col-0 WT and tga256 mutant plants. ROX19 strongly represses ROXY19 promoter activity in WT protoplasts but not in tga256 protoplasts (Figure 3.2A).

All three members of class II TGA transcription factors contain a conserved cysteine (Cys186). In order to investigate whether this cysteine is important for TGA2 to regulate the ROXY19 promoter activity, we constructed TGA2 mutant (TGA2 ${ }^{186} \mathrm{~S}$ ) by exchanging the cysteine residue with a serine. These TGA2 and TGA2C ${ }^{186} \mathrm{~S}$ effectors were expressed under the control of Arabidopsis UBQ10 promoter. In Col-0 and tga256 mutant protoplasts, both TGA2 and TGA2C ${ }^{186} \mathrm{~S}$ activate the ROXY19 promoter and this activation is repressed in the presence ROXY19 (Figure 3.2A). These results suggest that class II TGA factors are necessary to mediate ROXY19-mediated repression activity in protoplasts. However, the conserved cysteine of class II TGA factors is not important. 
A protoplast transient expression assay showed that a GFP-ROXY19 fusion protein is localized in the cytosol, similar to free GFP. Notably, the GFP-ROXY19 protein accumulates in the nucleus when coexpressed with TGA2 (Figure 3.2B). Since TGA2 and ROXY19 physically interact (Ndamukong et al., 2007) it may be speculated that TGA2 triggers the nuclear localization of GFP-ROXY19 through a piggyback mechanism. Alternatively, it might trap ROXY19 in the nucleus.

A

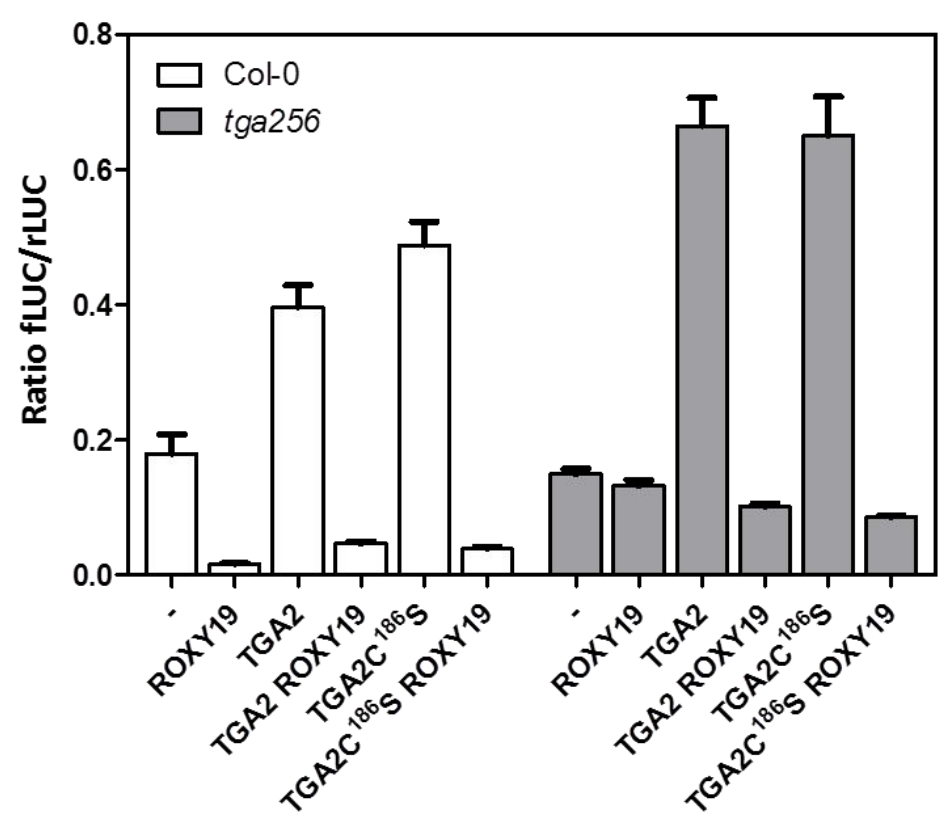

B

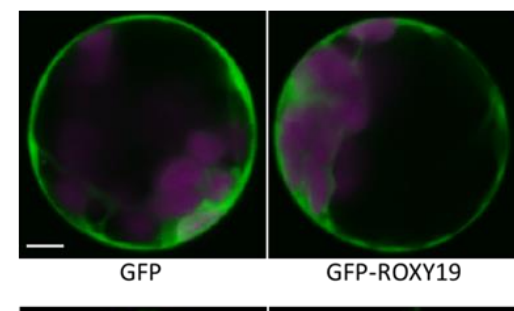

Figure 3.2 ROXY19 represses its own promoter activity in a class II TGA-dependent manner. (A) Transient expression assays with TGA2 and ROXY19 effectors and the ROXY19 promoter in Col-0 or tga256 mutant protoplasts. Expression of the ROXY19 promoter fused to the $f L u c$ was analyzed in transiently transformed mesophyll protoplasts in the presence of effector plasmids encoding ROXY19, TGA2 or TGA2C ${ }^{186} S$ under the control of the UBQ10 promoter. An empty plasmid was used when effector plasmid was absent (-). Luc activities were determined $16 \mathrm{~h}$ after transformation. Values are means of four replicates $( \pm S E)$. (B) Subcellular localization of GFP-ROXY19 fusion protein transiently expressed in Arabidopsis protoplasts. Different combinations of constructs expressing free GFP, HA tag, GFP-ROXY19 and HA-tagged TGA2 were transformed into Arabidopsis protoplasts. After $16 \mathrm{~h}$ incubation, GFP fluorescence and images were captured with a Leica confocal laser scanning microscope. The arrow denotes the presumed nucleus. Scale bar $=5 \mu \mathrm{m}$.

\subsubsection{The ROXY19 active site is not important for the repression activity}

Next, we examined whether the active site of ROXY19 was important to mediate the repression of ROXY19 promoter activity. The amino acids of the active site (CCMC) were replaced by SCMC, SSMS, ACMC or CPYC. As shown in Figure 3.3A, all active site mutant variants of ROXY19 still significantly repress ROXY19 promoter activity. In addition, the closest homologue of ROXY19 in Arabidopsis, ROXY18, also represses ROXY19 promoter activity regardless of the active site mutation. As shown before for the ORA59 promoter, the CPYC-type GRX, GRX370, does not repress the ROXY19 promoter (Figure 3.3A). 

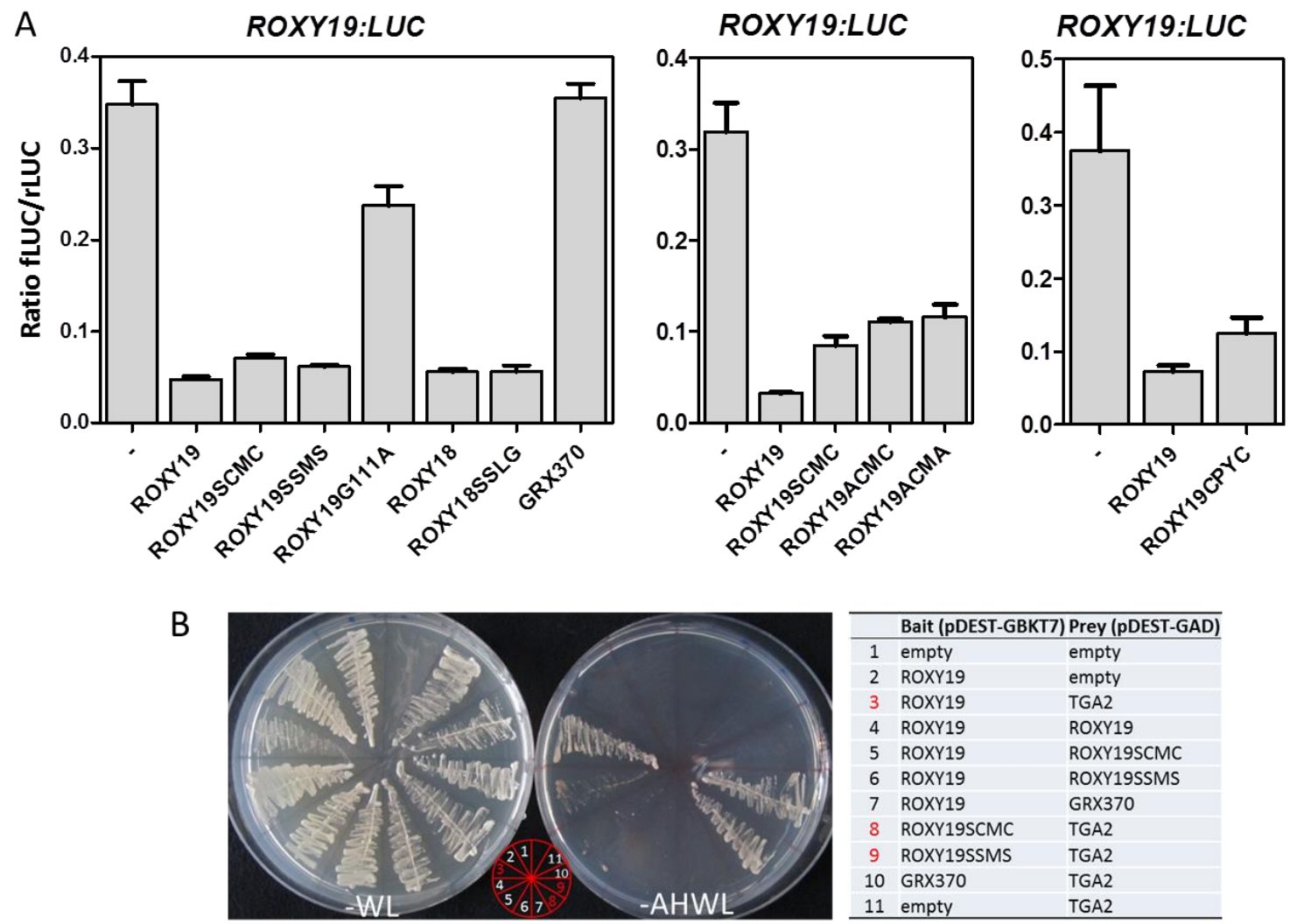

\begin{tabular}{cll}
\hline & \multicolumn{2}{l}{ Bait (pDEST-GBKT7) } \\
\hline 1 & empty & empty \\
2 & ROXY19 & empty \\
3 & ROXY19 & TGA2 \\
4 & ROXY19 & ROXY19 \\
5 & ROXY19 & ROXY19SCMC \\
6 & ROXY19 & ROXY19SSMS \\
7 & ROXY19 & GRX370 \\
8 & ROXY19SCMC & TGA2 \\
9 & ROXY19SSMS & TGA2 \\
10 & GRX370 & TGA2 \\
11 & empty & TGA2 \\
\hline
\end{tabular}

Figure 3.3 The ROXY19 active site is not important for repression of the ROXY19 promoter in transiently transformed protoplasts. (A) Transient expression assays with WT and mutant ROXY effectors and the ROXY19 promoter. Expression of the ROXY19 promoter fused to the fLuc was analyzed in transiently transformed mesophyll protoplasts in the presence of effector plasmids encoding WT and mutant variant CC-type ROXYs (ROXY18 and ROXY19) and CPYC-type glutaredoxin GRX370 under the control of the UBQ10 promoter. An empty plasmid was used when effector plasmid was absent (-). LuC activities were determined $16 \mathrm{~h}$ after transfection. Values are means of four replicates $( \pm S E)$. (B) Protein interaction between ROXY19 and TGA2 in yeast-two-hybrid assays. The bait plasmids encoding the indicated open reading frames fused to the GAL4 DNA binding domain (DBD); the prey plasmids encoding the indicated open reading frames fused to the GAL4 activation domain (AD); plasmids were co-transformed into yeast strain PJ69-4A. The combination of plasmids is indicated on the right. For interaction analysis, transformed yeasts were grown on selective media (A-H-W-L dropout); as control, yeasts were grown on non-selective media (W-L dropout).

In the GRX catalytic cycle, GRX itself is oxidized once the substrate becomes reduced. The oxidized GRX is reduced by binding to GSH. Mutation of an essential glycine (G110) in the putative GSH binding site of ROXY1 prevents the complement of the abnormal flower phenotype in roxy1 plants (Xing and Zachgo, 2008). ROXY19 with the corresponding glycine mutated into alanine (G111A) does not repress ORA59 promoter (Zander et al., 2012). Consistently, this ROXY19 mutant (ROXY19G111A) does not repress the ROXY19 promoter in protoplasts (Figure 3.3A), suggesting the GSH binding site is critical for ROXY19 function.

Yeast two hybrid assays showed that mutation of the GSH binding site (Zander et al., 2012) or the active site (Figure 3.3B) do not impede protein interaction between ROXY19 and TGA2. 


\title{
3.1.4 ROXY19 may recruit TOPLESS to repress target genes
}

The C-terminal hydrophobic ALWL motif of ROXY19 resembles the consensus sequence LXLXL of the so-called EAR (ERF-associated amphiphilic repression) motif that recruits transcriptional corepressors such as TOPLESS (TPL) and TOPLESS-RELATED PROTEINS (TPRs) (Kagale and Rozwadowski, 2011).

Dr. Joachim Uhrig (Department of Plant Molecular Biology and Physiology) found that ROXY19 interacts with TPL in yeast two hybrid assays and that the interaction requires an intact ALWL motif (Dr. Joachim Uhrig, personal communication). To test if the co-repressor TPL may be involved in ROXY19-mediated gene repression, we employed the protoplast transient expression assay. As shown in Figure 3.4A and B, ROXY19 strongly represses ROXY19 promoter activity, whereas TPL alone shows only a slightly negative effect. Co-expression of ROXY19 and TPL showed similar repression to that of ROXY19 alone (Figure 3.4A and B). A truncated variant TPL351 (Dr. Joachim Uhrig, personal communication), which includes only the $\mathrm{N}$-terminal 351 amino acids and which still interacts with ROXY19 in yeasts, significantly intercepted ROXY19 repression activity (Figure 3.4B). It is envisioned that association of the N-terminal domain of TPL with ROXY19 does lead to repression but that it blocks the access of the endogenous TPL. When replacing the C-terminal ALWL motif by an EAR motif of SUPERMAN protein (ROXY19EAR, Dr. Joachim Uhrig, personal communication) we observed a strong repression suggesting that the ALWL motif and the EAR motif have the same function, i.e. recruitment of TPL (Figure 3.4C).
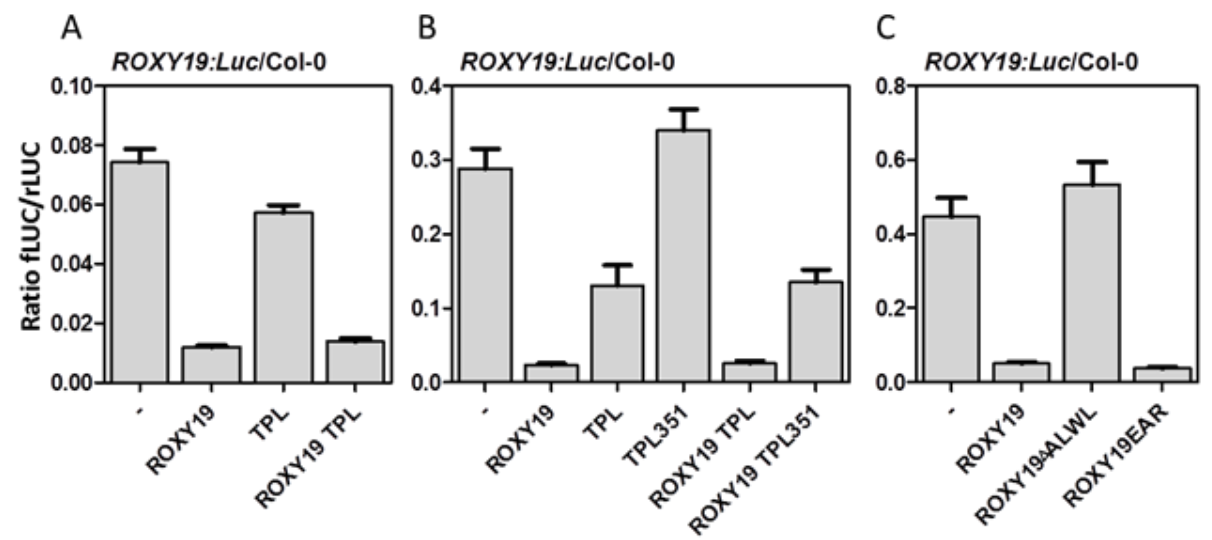

\begin{abstract}
Figure 3.4 ROXY19 may recruit TOPLESS (TPL) to repress target genes. (A) Transient expression assays with ROXY19 and TPL effectors and ROXY19 promoter in Col-0 protoplasts. Expression of the ROXY19 promoter fused to the $f L u c$ was analyzed in transiently transformed mesophyll protoplasts in the presence of effector plasmids encoding ROXY19 or TPL under the control of the UBQ10 promoter. (B) Transient expression assays with ROXY19, TPL and the truncated TPL351 effectors and the ROXY19 promoter in Col-0 protoplasts. (C) Transient expression assays with WT and mutant ROXY19 effectors and ROXY19 promoter in Col-0 protoplasts. ROXY19EAR contains the EAR motif (LELRLDL) of Arabidopsis SUPERMAN protein that was used to replace the ALWL motif. An empty plasmid was used when effector plasmid was absent (-). Luc activities were determined $16 \mathrm{~h}$ after transformation. Values are means of four replicates $( \pm \mathrm{SE})$.
\end{abstract}

These preliminary results indicate that TPL may be deployed by ROXY19 to repress target gene expression. Since TPL alone has a weak effect on ROXY19 promoter activity, it is likely that ROXY19 is a limiting factor that inactivates TGA-mediated activation of the ROXY19 promoter by recruitment of TPL. 


\subsubsection{ROXY19 represses JA-induced ROXY19 expression in transgenic plants}

So far, our results showed that ROXY19 represses its own promoter in a manner that requires TGA factors and possibly the interaction with TPL. However, the repression mechanism does not require the active site or the presence of the conserved cysteine in the coding region of at least TGA2.

Next we aimed to investigate whether the mechanism of repression involves similar molecular components if the target promoter is not part of a transiently transformed plasmid but rather located in its native locus in the chromatin. First, we tested whether ectopically expressed ROXY19 represses its own promoter. We examined JA-induced endogenous ROXY19 expression in a previously established ROXY19 overexpression line (ROXY19OE) in which the expression of ROXY19 is driven by the CaMV35S promoter (Zander et al., 2012). Figure 3.5 showed that JA-induced endogenous ROXY19 is strongly repressed in ROXY19 overexpression plants. This result was reproduced with more independent 35S:HA-ROXY19 transgenic lines (See section 3.1.7 Figure 3.6A and Supplemental Figure S3A).

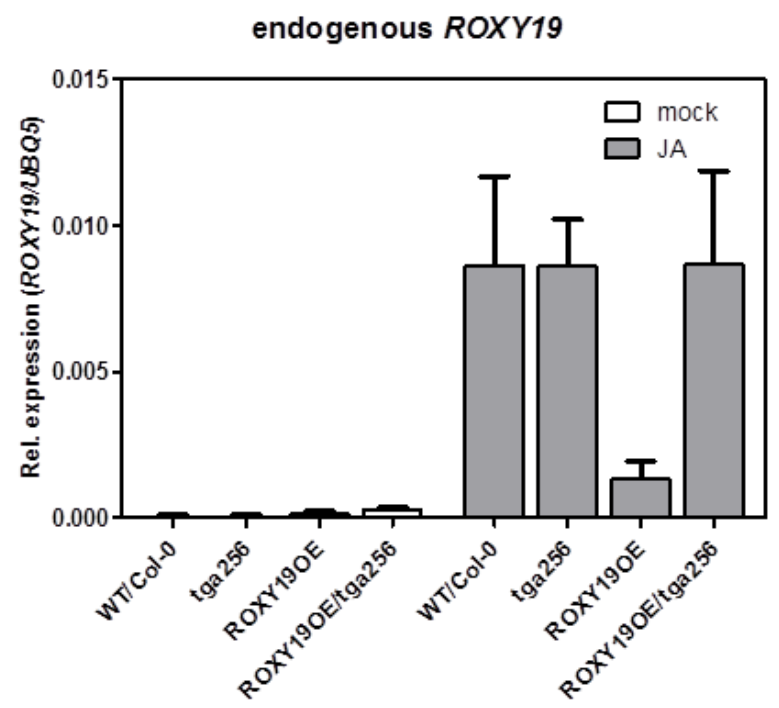

Figure 3.5 Class II TGA factors are required for ROXY19 to repress JA-induced ROXY19 expression in transgenic plants. $\mathrm{qRT}-\mathrm{PCR}$ analysis of endogenous ROXY19 expression in WT Col-0, tga256, ROXY19OE and ROXY19OE/tga256 plants treated with JA. Arabidopsis seeds were germinated and grown on soil for four weeks, and subsequently treated with volatile JA (MeJA) in glass aquaria. After $8 \mathrm{~h}$ of treatment, plant leaves were harvested for RNA extraction, endogenous ROXY19 transcripts were analyzed by qRT-PCR. The mean values $( \pm S E)$ from 6-8 independent replicates (one pot with one plant as a biological replicate) are shown.

\subsubsection{ROXY19 cannot repress JA-induced ROXY19 expression in the tga256 mutant}

The ROXY19 overexpression line (ROXY19OE) was crossed with the tga256 mutant to generate a homozygous line ROXY19OE/tga256 (AG Gatz). Since ROXY19 expression cannot be induced by SA in the tga256 mutant, we induced the gene with JA, which induces the ROXY19 gene independently of class II TGAs (Köster et al., 2012 and Figure 3.5). qRT-PCR analysis revealed that ectopic ROXY19 expression represses JA-induced endogenous ROXY19 transcription in Col-0 but not in tga256 mutant background (Figure 3.5).

\subsubsection{The ROXY19 active site is required for repression of its target promoter in plants}

In order to investigate the importance of the conserved active site for ROXY19-mediated repression of genes in their natural chromatin context, we generated Arabidopsis transgenic plants constitutively expressing HA-tagged WT (ROXY19CCMC) or active site mutant (ROXY19SSMS) ROXY19 under the control of the CaMV35S promoter. These transgenic plants were named 35S:HAROXY19CCMC and 35S:HA-ROXY19SSMS, respectively. Western blot assay showed that all these lines express HA-ROXY19 protein to similar levels (Figure 3.6C), except for a heterozygous line CCMC N\#18 
and a homozygous line SSMS N\#14. Therefore, these lines were not included in hormone induction experiment.
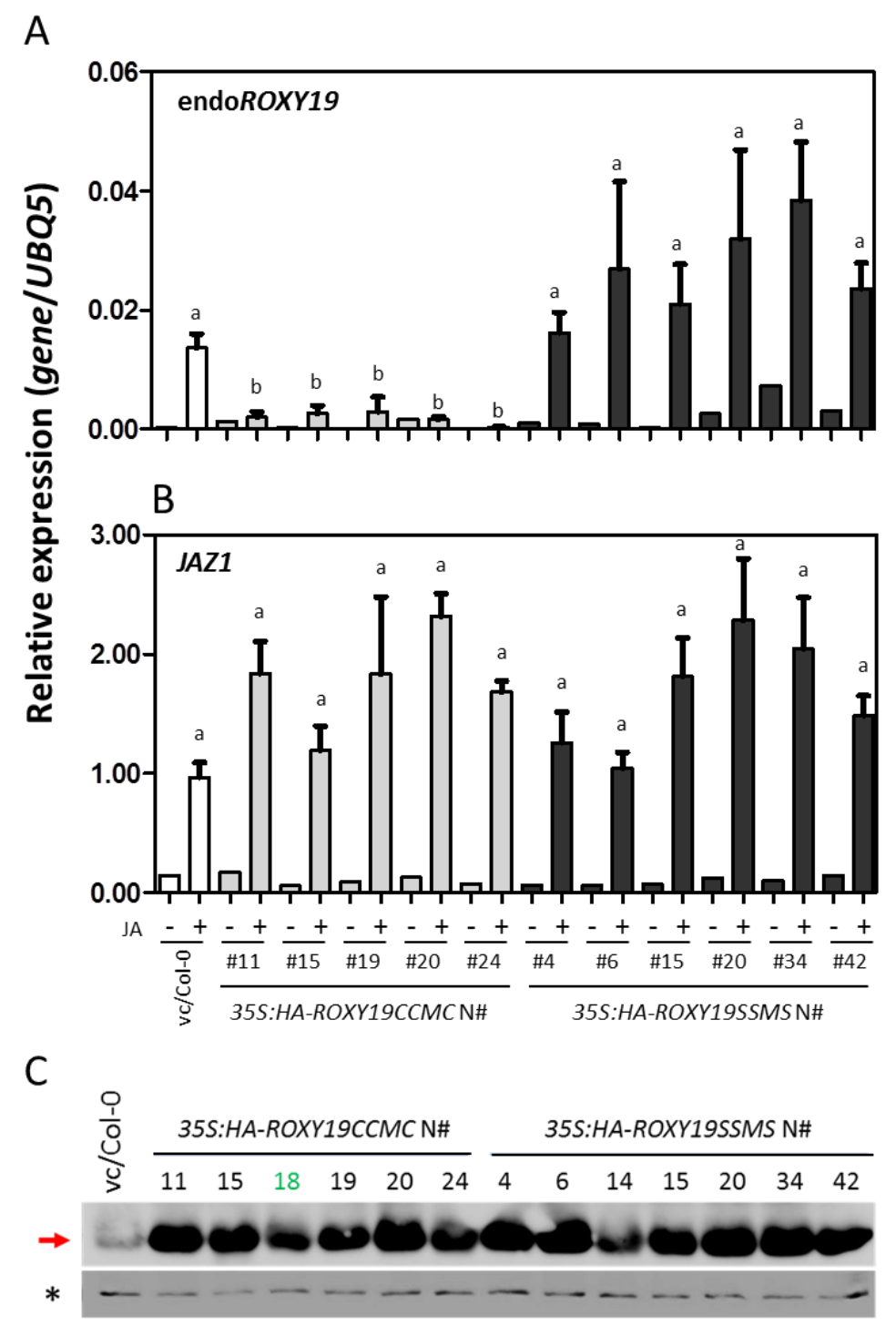

Figure 3.6 Repression of JA-induced ROXY19 expression by ROXY19 depends on a functional active site. (A) qRT-PCR analysis of endogenous ROXY19 expression in 35S:HA-ROXY19CCMC and 35S:HAROXY19SSMS transgenic plants after JA treatment. Homozygous Arabidopsis seeds from the T2 generation were germinated and grown on soil for 4 weeks, and subsequently treated with volatile JA (MeJA) in glass aquaria. After $10 \mathrm{~h}$ of treatment, plant leaves were harvested for RNA or protein extraction, endogenous ROXY19 transcripts were analyzed by qRT-PCR. (B) qRT-PCR analysis of JAZ1 expression in ROXY19 transgenic plants after JA treatment. Samples are the same as used in Figure 3.6A. The mean values $( \pm S E)$ from four independent replicates (one pot with one plant as one biological replicate) of JA-treated samples are shown. One replicate of the corresponding mock sample is shown. Different letters in Figure 3.6A indicate significant difference among genotypes after treatment (Student's $t$-test, $p<0.05)$. The same letter Figure 3.6B indicates no significant difference among genotypes after treatment (Student's $t$-test, $p<0.05$ ). White, gray and black bars indicate empty vector, WT and active site mutant ROXY19 transformed Col-0 samples, respectively. (C) Western blot analysis of the ROXY19 transgenic lines using the antibody against the HA tag. Protein was extracted from mock plant samples used for RNA extraction in Figure 3.6A. Asterisk and arrow indicate unspecific bands, which serve as loading control, and specific bands, respectively. 
After JA treatment of 30-days-old soil-grown plants, all 35S:HA-ROXY19CCMC lines (lines CCMC N\#11, $\# 15, \# 19, \# 20$ and \#24) but not 35S:HA-ROXY19SSMS lines (lines SSMS N\#4, \#6, \#15, \#20, \#34 and \#42) were compromised with respect to JA-induced endogenous ROXY19 expression (Figure 3.6A). As control for the successful JA treatment, expression of the JA-responsive JAZ1 was monitored (Figure 3.6B). The results suggest that the active site of ROXY19 is important for repressing endogenous ROXY19 expression. These results differ from the observations made in transiently transformed protoplasts.

Our previously generated transgenic plants showed the same results. In transgenic lines 35S:HAROXY19CCMC\#8 and \#12 JA-induced expression of endogenous ROXY19 is strongly repressed which is not the case in lines 35S:HA-ROXY19SSMS\#9 and \#18 (Supplemental Figure S3A). As control, all transgenic plants showed induction of the JA-responsive LOX2 gene to a similar level when compared to Col-0 plants (Supplemental Figure S3B). Western blot analysis showed that all these transgenic lines express similar levels of HA-ROXY19 protein (Supplemental Figure S3C).

Next, we exchanged the active site of ROXY19 from CC-type into CPYC-type (ROXY19CPYC) and generated Arabidopsis transgenic plants, which were named 35S:HA-ROXY19CPYC. Among four transgenic lines, three lines (lines CPYC\#2, \#10 and \#15) showed protein expression to the same level as the previously generated 35S:HA-ROXY19CCMC\#8 line (Figure 3.7B). After JA treatment of soilgrown plants, the induction of ROXY19 expression was compromised in lines 35S:HA-ROXY19CPYC\#9 and \#15 and totally abolished in lines 35S:HA-ROXY19CPYC\#2 and \#10 (Figure 3.7A).

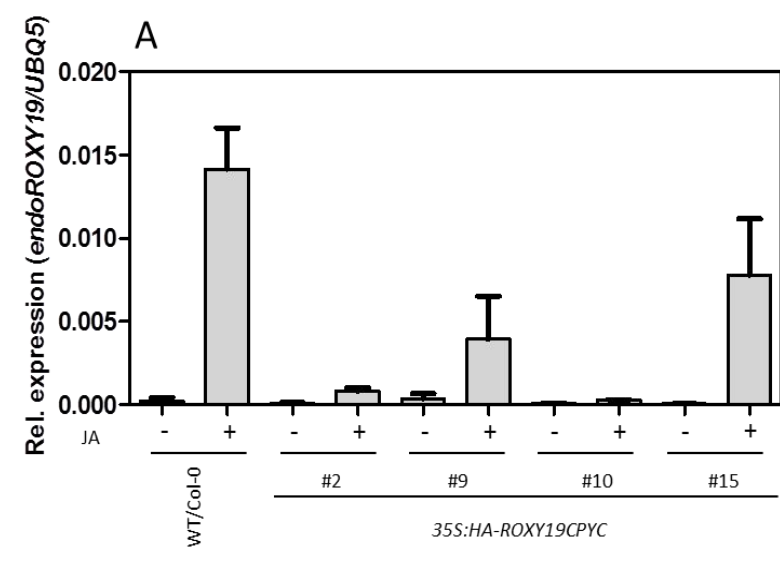

B

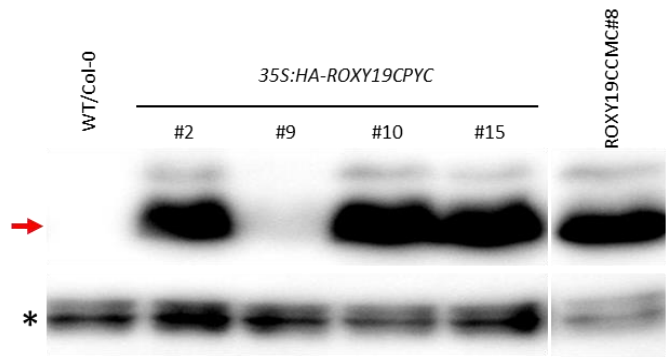

Figure 3.7 ROXY19 with a CPYC-type active site represses JA-induced ROXY19 expression in transgenic plants. (A) qRT-PCR analysis of endogenous ROXY19 expression in Col-0 and 35S:HA-ROXY19CPYC transgenic plants after JA-treatment. Homozygous Arabidopsis seeds from the $\mathrm{T} 2$ generation were germinated and grown on soil pot for four weeks, and subsequently treated with volatile JA (MeJA) in glass aquaria. After $10 \mathrm{~h}$ of treatment, plant leaves were harvested for RNA or protein extraction, endogenous ROXY19 transcripts are analyzed by $q R T-P C R$. The mean values ( $\pm S E$ ) from four independent replicates (one pot with one plant as one biological replicate) are shown. (B) Western blot analysis of the 35S:HA-ROXY19CPYC transgenic plants using antibody against HA tag. Asterisk and arrow indicate unspecific bands, which served as loading control, and specific bands, respectively. All samples were analyzed in the same gel, there is one lane removed between CPCY\#15 and CCMC\#8 in the image. 


\subsection{Characterization of ROXY19 knock-out mutant}

\subsubsection{ROXY19 represses JA-induced CYP81D11 expression}

The CYP81D11 gene, which contains a functional as-1-like element, is induced by JA in a class II TGAdependent manner (Köster et al., 2012). Since ectopically expressed ROXY19 represses JA-induced ROXY19 expression, we tested whether JA-induced CYP81D11 is repressed in our 35S:HA-ROXY19 plants after JA treatment. Indeed, the CYP81D11 gene is repressed in four 35S:HA-ROXY19CCMC lines (lines CCMC N\#11, \#15, \#19 and \#20) (Figure 3.8A). The capacity to repress CYP81D11 requires the active site of ROXY19 (Figure 3.8A).

\subsubsection{JA-induced CYP81D11 expression is not altered in ROXY19 knock-out mutant}

Since we found that ectopically expressed ROXY19 can repress JA-induced genes, such as its own gene and CYP81D11 gene and since ROXY19 is the only ROXY that is induced by JA, we expected that the CYP81D11 gene would be hyper-induced in the ROXY19 loss-of-function mutant after JA treatment.

Since no T-DNA insertion mutant of ROXY19 in the Col-0 background is available we worked with the transposon-tagged line roxy19DS, in which a Ds transposon is inserted 45 bp upstream of the ROXY19 start codon in the Nossen background (Ndamukong, 2006 and Ito et al., 2002). qRT-PCR analysis revealed that salt $(\mathrm{NaCl})$-induced $R O X Y 19$ expression in root tissues was not detected in the roxy19DS plants (Figure 3.8B), indicating this line is a knock-out mutant. However, expression of CYP81D11 was induced to a similar level by JA in Nossen and roxy19DS plants (Figure 3.8C).

\subsubsection{Microarray analysis of roxy19DS mutant}

We used microarray analysis to identify genes that are differentially expressed in roxy19DS and WT Nossen plants. The WT Col-0 and 35S:HA-ROXY19 transgenic plants were also included for further analysis (See section 3.3.1). We chose to grow the plants under unstressed conditions in order to be able to identify target genes unhampered by the variability of the applied stress. In essence we expected to identify reciprocal changes in the expression of genes in the loss-of-function and the gain-of-function plants assuming that uninduced levels of ROXY19 would already influence gene expression. A similar approach had been previously successful for the identification of target genes of the GRAS protein SCL14 (Fode et al., 2008). Arabidopsis seeds of each background were germinated side by side and grown vertically on MS-plates for two weeks. Subsequently, the whole seedlings (shoots and roots) were collected for microarray analysis with the Arabidopsis GeneChip ${ }^{\circledR}$ Gene 1.0 ST Arrays (Affymetrix) (Figure 3.8D). Four biological repeats were performed with independently grown plants.

A principle component analysis of the microarray data showed that the expression profile of roxy19DS is distinct from that of WT Nossen (Figure 3.8E). However, it has to be considered that the DS transposon line roxy19DS contains part of the Landsberg genome (Ndamukong, 2006). Therefore, we first compared the expression profile of the Nossen ecotype to that of Col-0 ecotype and selected the genes that expressed to a similar degree (fold change<1.5) in both ecotypes. This step excluded genes that show an ecotype-specific expression pattern and might be due to the difference between Nossen and the Nossen/Landsberg hybrid. Because ROXY19 specifically represses targeted genes of class II TGA factors, we were interested in genes that were up-regulated in roxy19DS mutant. However, none of the genes (PDF1.2, ORA59, CYP81D11 and ROXY18), which are down-regulated in the ROXY19 overexpressing lines was up-regulated in the mutant. No GO term was enriched in genes 
down- or up-regulated in roxy19DS mutant according the method and criteria (more than $5 \%$ genes) used for analysis of genes down-regulated in CCMC\#8 (See section 3.3.1 Figure 3.10A). Nevertheless, top 100 up-regulated and down-regulated genes (fold change $>1.5, p$ value $<0.05$ ) were selected and listed in Supplemental Table S2.

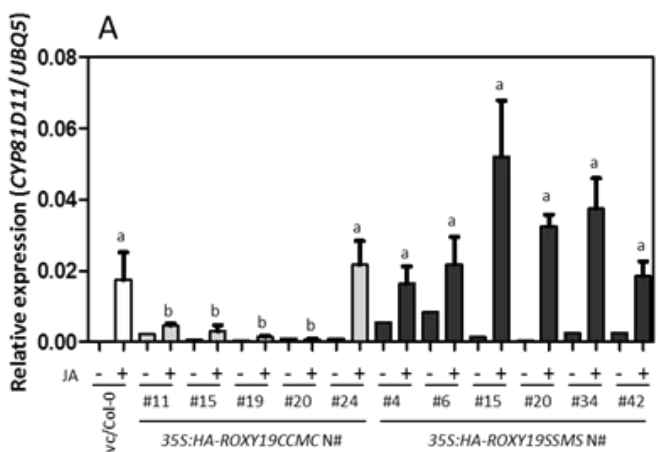

D

grown plants on MS plates

collect whole seedlings and RNA extraction with TRIzol

microarray and meta-analysis

confirmation of the results
$E$

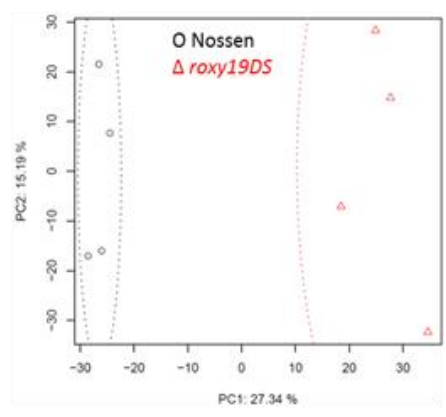

B

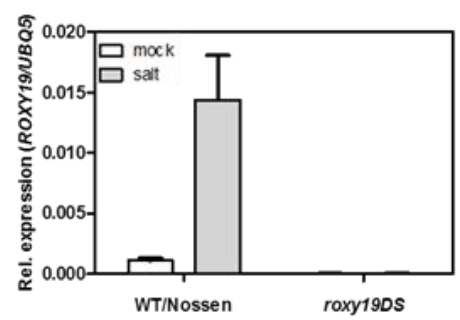

F

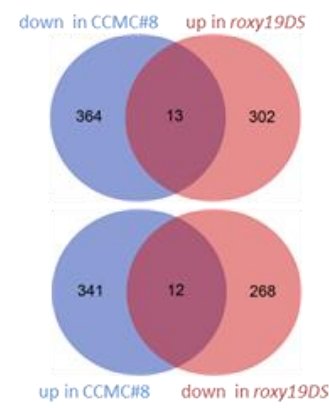

C

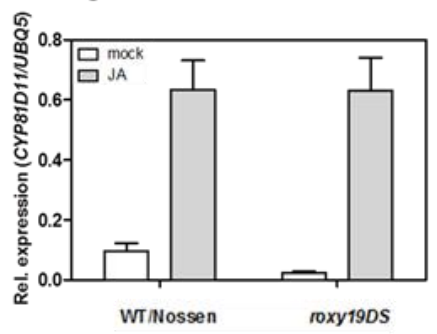

G

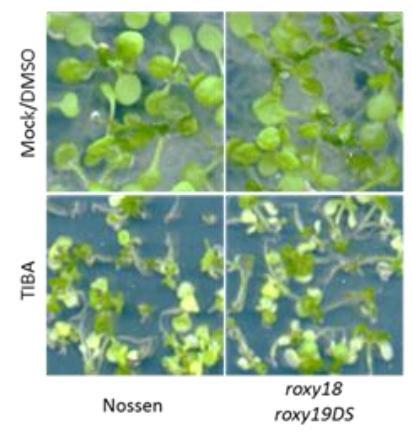

Figure 3.8 Characterization of ROXY19 gain- and loss-of-function plants. (A) qRT-PCR analysis of CYP81D11 expression in ROXY19 transgenic plants after JA treatment. Samples are the same as used in Figure 3.6A. The same letter indicates no significant difference among genotypes after treatment (Student's t-test, $p<0.05$ ). White, gray and black bars indicate empty vector, WT and active site mutant ROXY19 transformed samples, respectively. (B) qRT-PCR analysis of ROXY19 expression in Nossen and roxy19DS mutant plants after salt $(\mathrm{NaCl})$ treatment. Nossen and roxy19DS mutant plants were grown in hydroponic jars for two weeks, and subsequently treated with mock and $150 \mathrm{mM}$ salt ( $\mathrm{NaCl}$ ). After $6 \mathrm{~h}$ of treatment, root tissues were harvested for RNA extraction. ROXY19 transcripts were analyzed with qRT$P C R$. The mean values $( \pm S E$ ) from five independent replicates (one jar with approximately 100 seedlings as one biological replicate) are shown. (C) qRT-PCR analysis of CYP81D11 expression in Nossen and roxy19DS mutant plants after JA treatment. Nossen and roxy19DS mutant plants were grown on soil for four weeks and subsequently treated with mock and volatile JA (MeJA) in glass aquaria. The mean values ( \pm SE) from 8 independent replicates (one pot with one plant as one biological replicate) are shown. (D) Experimental procedures of microarray analysis with ROXY19 gain- and loss-of-function mutants and control plants. Arabidopsis seeds were germinated and grown vertically on MS-plates for two weeks, and subsequently $\sim 50$ seedlings of each genotype were harvested for RNA extraction with TRIzol solution. Four biological replicates were performed. RNA sample were analyzed with Affymetrix GeneChips ${ }^{\circledR}$ Gene 1.0 ST Arrays. (E) Principal components analysis of microarray data from Nossen and roxy19DS. (F) Venn diagram shown genes that are reciprocally regulated in roxy19DS and CCMC\#8 plants. Genes that were down- or upregulated in roxy19DS and CCMC\#8 were used (FC>1.5, $p$ value<0.05). Overlapping genes and their expression are shown in Supplemental Table S3. (G) Growth phenotype of Nossen and roxy18 roxy19DS double mutant plants on MS-plates containing DMSO (mock) or TIBA. Arabidopsis seeds were germinated and grown on MS-plates containing DMSO (mock) or 50 $\mu \mathrm{M}$ TIBA for two weeks. 


\subsection{ROXY19 represses detoxification pathway genes}

\subsubsection{Isolation of ROXY19-regulated genes by microarray analysis}

So far, we have identified the potential target genes of ROXY19 through educated guesses. We were able to support our hypothesis by using plants ectopically expressing ROXY19, but so far loss-offunction evidence is lacking and the microarray analysis of WT Nossen and the roxy19DS mutant under non-inducing conditions had not given any hint on the endogenous function of ROXY19. The identification of further target genes of ROXY19 might give us a hint under which experimental conditions we might detect a loss-of-function phenotype. Therefore, we carried out transcriptional profiling analysis of lines 35S:HA-ROXY19CCMC\#8 and \#12, line 35S:HA-ROXY19SSMS\#9, line 35S:HAGRX370\#1 and corresponding WT Col-0. Growth conditions (axenically grown seedling) and type of harvest (roots and shoots) were done as described in the previous section for the analysis of Nossen and roxy19DS mutant (See section 3.2.3).

A principle component analysis (PCA) revealed that expression profiles of line 35S:HAROXY19SSMS\#9 was much closer to that of Col-0 and defined a group that was distinct from lines 35S:HA-ROXY19CCMC\#8 and \#12 (Figure 3.9A). This result suggests that ROXY19SSMS is dysfunctional in planta. To check the specific repression of genes in different transgenic lines, genes that were down-regulated in CCMC\#8, CCMC\#12, SSMS\#9 and GRX370\#1 when compared to WT Col0 were selected with a cut-off of fold change more than 1.5 and $p$ value less than 0.05 (FC>1.5, $p$ value<0.05). This analysis identified 398 genes for CCMC\#8, 296 for CCMC\#12, 130 for SSMS\#9 and 173 for GRX370\#1, respectively. A Venn diagram analysis showed that 151 and 43 genes are downregulated in both CCMC\#8 and CCMC\#12 plants, and CCMC\#8 and GRX370\#1 plants, respectively (Figure 3.9B).

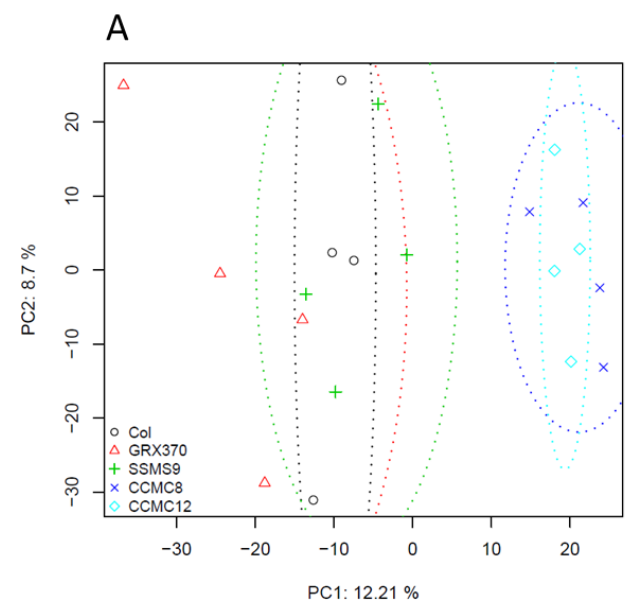

B

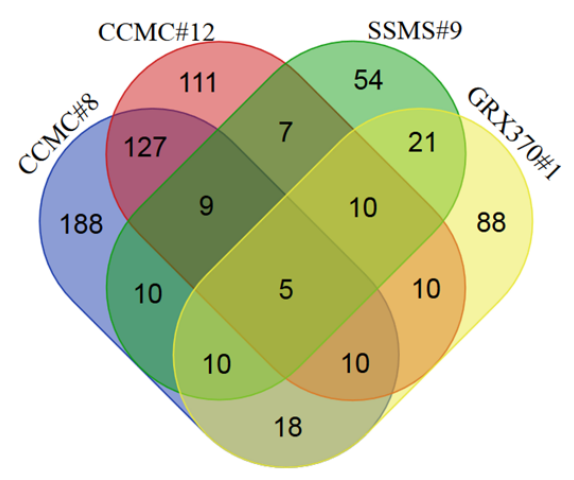

Figure 3.9 Microarray analysis of ROXY19 overexpression lines. (A) Principal components analysis of microarray data. (B) Venn diagram analysis of down-regulated genes in transgenic plants. Genes that were down-regulated in CCMC\#8, CCMC\#12, SSMS\#9 and GRX370\#1 when compared to Col-0 were selected (FC>1.5, $p$ value<0.05). This analysis identified 398 genes for CCMC\#8, 296 for CCMC\#12, 130 for SSMS\#9 and 173 for GRX370\#1, respectively. AGI (Arabidopsis Genome Initiative) codes of these genes were used for Venn diagram sorting.

Next, we tested, whether genes that are repressed or induced in the overexpressing lines were reciprocally regulated in the knock-out mutant. A Venn diagram analysis showed that 13 genes are up-regulated in roxy19DS and down-regulated in CCMC\#8 and 12 genes reversely (Figure 3.8F, 
Supplemental Table S3). Interestingly, the TGACGTMA ( $\mathrm{M}=\mathrm{A}$ or $\mathrm{C}$ ) motif was found in the promoter regions of three genes (AT2G18230/AtPPa2, AT2G21187/other RNA and AT4G34000/ABF3) which are up-regulated in roxy19DS and down-regulated in CCMC\#8. It is interesting to verify whether these three genes are direct targets of the ROXY19-TGA-module. No TGACGT motif was found in the promoter of genes that are down-regulated in roxy19DS and up-regulated in CCMC\#8, suggesting their expression is indirectly influenced in those plants.

Since the microarray analysis of the knock-out plants did not reveal any clue on the endogenous function of ROXY19, we focused on those genes that were significantly altered in the 35S:ROXY19CCMC\#8 line. Due to the fact that the ALWL motif is important for ROXY19 function and that it mediates the interaction with the transcriptional co-repressor TPL, we concentrated our interest on 337 genes that were significantly repressed (FC>1.5, $p$ value $<0.05)$ in CCMC\#8 but not in SSMS\#9 and GRX370\#1 when compared to Col-0 plants. These genes are listed in Supplemental Table S1. From ROXY19-repressed genes, a gene ontology (GO) analysis with the agriGO platform (Du et al., 2010) revealed that the following biological terms were over-represented (Figure 3.10A).

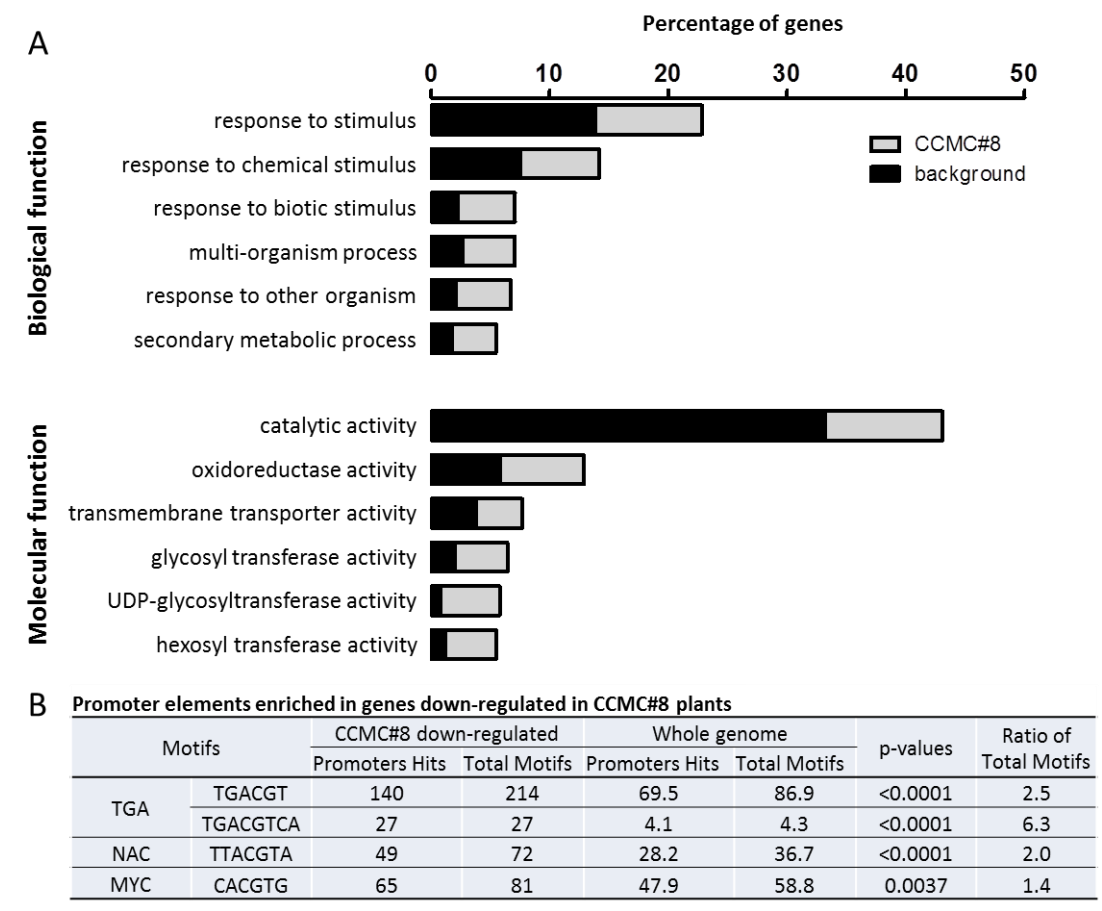

Figure 3.10 Meta-analysis of genes down-regulated in 35S:HA-ROXY19CCMC\#8 plants. (A) Gene Ontology (GO) over-representation analysis of 337 putative target genes which show reduced expression (FC>1.5, $p$-value<0.05) in 35S:HA-ROXY19\#8 but not in 35S:HA-ROXY19SSMS\#9 plants. Black bars indicate the percentage of genes of each GO term found within the group of all annotated genes of the Arabidopsis genome. Gray bars indicate the percentage of genes of each GO term found within the subgroup of genes repressed CCMC\#8 plants. Only GO terms encompassing more than 17 genes (5\%) are shown. (B) Promoter elements enriched in genes negatively affected in 35S:HAROXY19CCMC\#8 plants. The occurrence of enriched motifs was determined in the 1-kb sequences upstream of the $5^{\prime}$-untranslated regions using Motif Mapper (Berendzen et al., 2012).

The GO term "response to stimulus" and its sub-GO term "response to chemical stimulus" revealed the most significant enrichment within the GO domain "biological process". Within the GO domain "molecular function", the GO term "catalytic activity" and its sub-GO terms "oxidoreductase activity" (phase I of the detoxification process) and "transmembrane transporter activity" (phase III of the 
detoxification process) were enriched. Moreover, the GO term "transferase activity, transferring glycosyl groups" (phase II of the detoxification process) was overrepresented.

Among transcription factors repressed in CCMC\#8, five (ATAF1/ANAC002, ANAC014, NAC032, ANAC041 and ANAC102) belong to the NAC [for NAM (no apical meristem), ATAF (Arabidopsis thaliana activating factor), CUC (cup-shaped cotyledon)] family. The expression of ATAF1 and ANAC102 is also inducible by the xenobiotic chemical benzoxazolin-2(3H)-one (BOA) (Baerson et al., 2005).

Subsequently, a motif-based sequence analysis performed using Motif Mapper (Berendzen et al., 2012) revealed that TGA- and NAC-binding sites are the most enriched motifs in the promoters (1000 bp upstream of the $5^{\prime}$-untranslated regions) of ROXY19 repressed genes (Figure 3.10B). Notably, the TGACGTCA palindrome is more than six-fold enriched with 27 hits in the repressed genes. This already indicates that ROXY-mediated repression operates at TGA-regulated promoters.

\subsubsection{ROXY19 represses detoxification pathway genes}

In order to confirm the novel results from our microarray analysis, we analyzed the expression of specific detoxification genes after xenobiotic treatment by qRT-PCR. Previous analysis had shown that the electrophilic halogenated phenol TIBA (2,3,5-Triiodobenzoic acid) induces genes of the detoxification pathway, many of them in a class II TGA-dependent manner (Fode et al., 2008). TIBAinduced expression of three detoxification pathway genes (CYP81D11, OPR2 and ANACO32) is strongly repressed in 35S:HA-ROXY19CCMC plants (lines CCMC\#8 and \#12) but not in 35S:HAROXY19SSMS plants (lines SSMS\#9 and \#18) (Figure 3.11A). In concert with gene expression results, both 35S:HA-ROXY19CCMC lines were more sensitive to TIBA on MS-plates (Figure 3.11B).
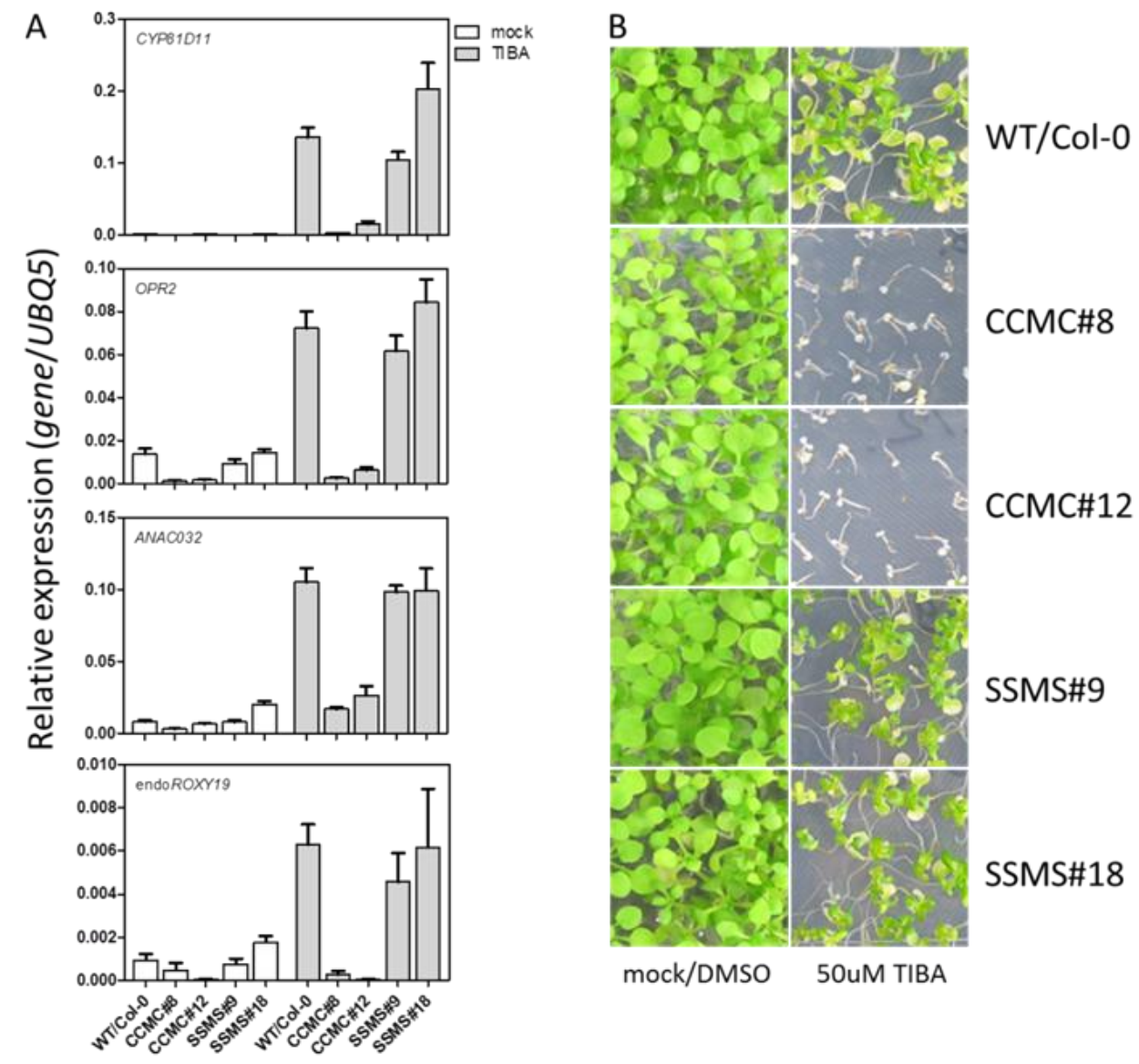
Figure 3.11 ROXY19 represses detoxification pathway genes and renders plant more sensitive to TIBAtreatment. (A) qRT-PCR analysis of detoxification pathway genes and endogenous ROXY19 expression in 35S:HA-ROXY19CCMC and 35S:HA-ROXY19SSMS transgenic plants. Arabidopsis seeds were germinated on soil and grown for four weeks, and subsequently sprayed with DMSO (mock) or 0.1mM TIBA. After $10 \mathrm{~h}$ of treatment, plant leaves were harvested for RNA extraction and CDNA synthesis, CYP81D11, OPR2, ANAC032 and endogenous ROXY19 transcripts were analyzed with qRT-PCR. The mean values ( \pm SE) from 4-5 independent replicates (one pot with one plant as one biological replicate) are shown. (B) Growth phenotype of WT Col-0 and different ROXY19 overexpression lines on TIBA-containing plates. Arabidopsis seeds were germinated and grown on MS-plates containing DMSO (mock) or 50 MM TIBA for two weeks.

Quantification of the chlorophyll content also showed that the transgenic line XVE\#9, which expresses ROXY19 under the control of a $\beta$-estradiol-inducible promoter (See section 3.5 Figure 3.17A and B), is more sensitive to TIBA than Col-0 in the presence of $\beta$-estradiol (Figure 3.12A and $B$ ).

A

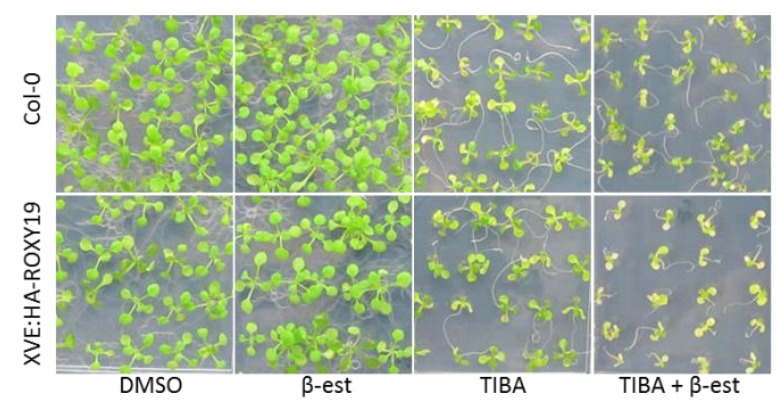

B

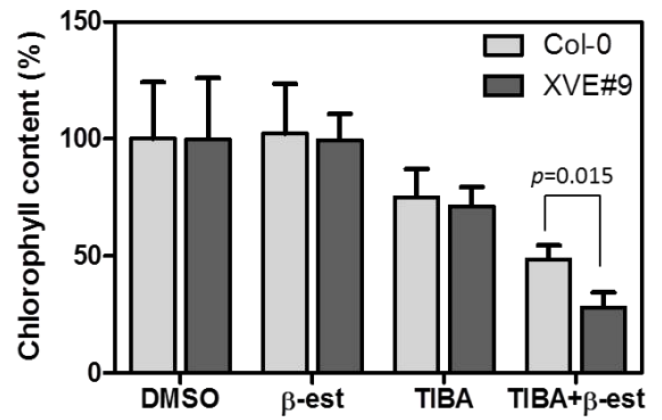

Figure 3.12 $\beta$-estradiol-induced ROXY19 expression renders Arabidopsis transgenic plants more sensitive to TIBA. (A) Growth phenotype of Arabidopsis plants grown on MS-plates. WT Col-0 and XVE\#9 transgenic plants were germinated and grown on MS-plates containing DMSO (mock), $\beta$-estradiol, TIBA and $\beta$-estradiol plus TIBA. The images were taken after 2 weeks of growth. (B) Quantitative analysis of total chlorophyll content in leaves of the indicated genotypes grown on MS-plates as shown in Figure 3.12A. Data are mean \pm SEM of three biological replicates. $p$-value indicates significant difference between genotypes after treatment with TIBA and $\beta$-estradiol (Student's $t$-test).

A growth assay with another set of independently generated transgenic lines showed that four 35S:HA-ROXY19CCMC lines (lines CCMC\#11, \#15, \#19 and \#20) are more sensitive to TIBA, while all seven 35S:HA-ROXY19SSMS lines (lines SSMS\#4, \#6, \#14, \#15, \#20, \#34 and \#42) are affected to the same degree as Col-0 (Figure 3.13).

Next, the TIBA-induced marker gene CYP81D11 expression was analyzed in the 35S:HA-ROXY19CPYC transgenic plants (Section 3.1.7). TIBA-induced CYP81D11 expression was repressed in lines CPYC\#2 and \#10 (Figure 3.14A). However, TIBA-induced CYP81D11 was only compromised in line CPYC\#15 which expressed relative high protein amounts (Section 3.1.7, Figure 3.7B). Consistently, the growth assay revealed that lines (35S:HA-ROXYCPYC\#2 and \#10), which repress CYP81D11 expression, were also more sensitive to TIBA (Figure 3.14C). 

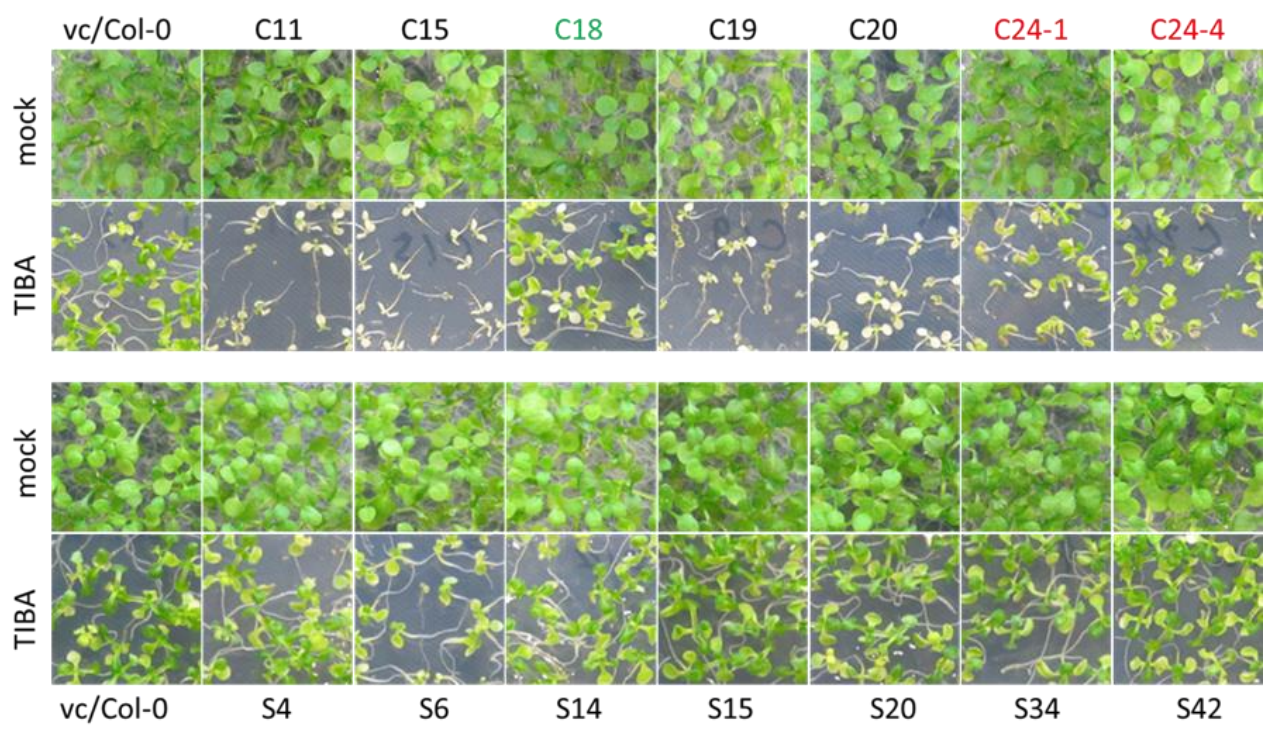

Figure 3.13 ROXY19 overexpression plants are more sensitive to xenobiotic TIBA. Like in Figure 3.11B, Arabidopsis seeds of the indicated lines were germinated and grown on MS-

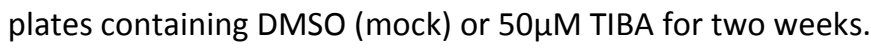
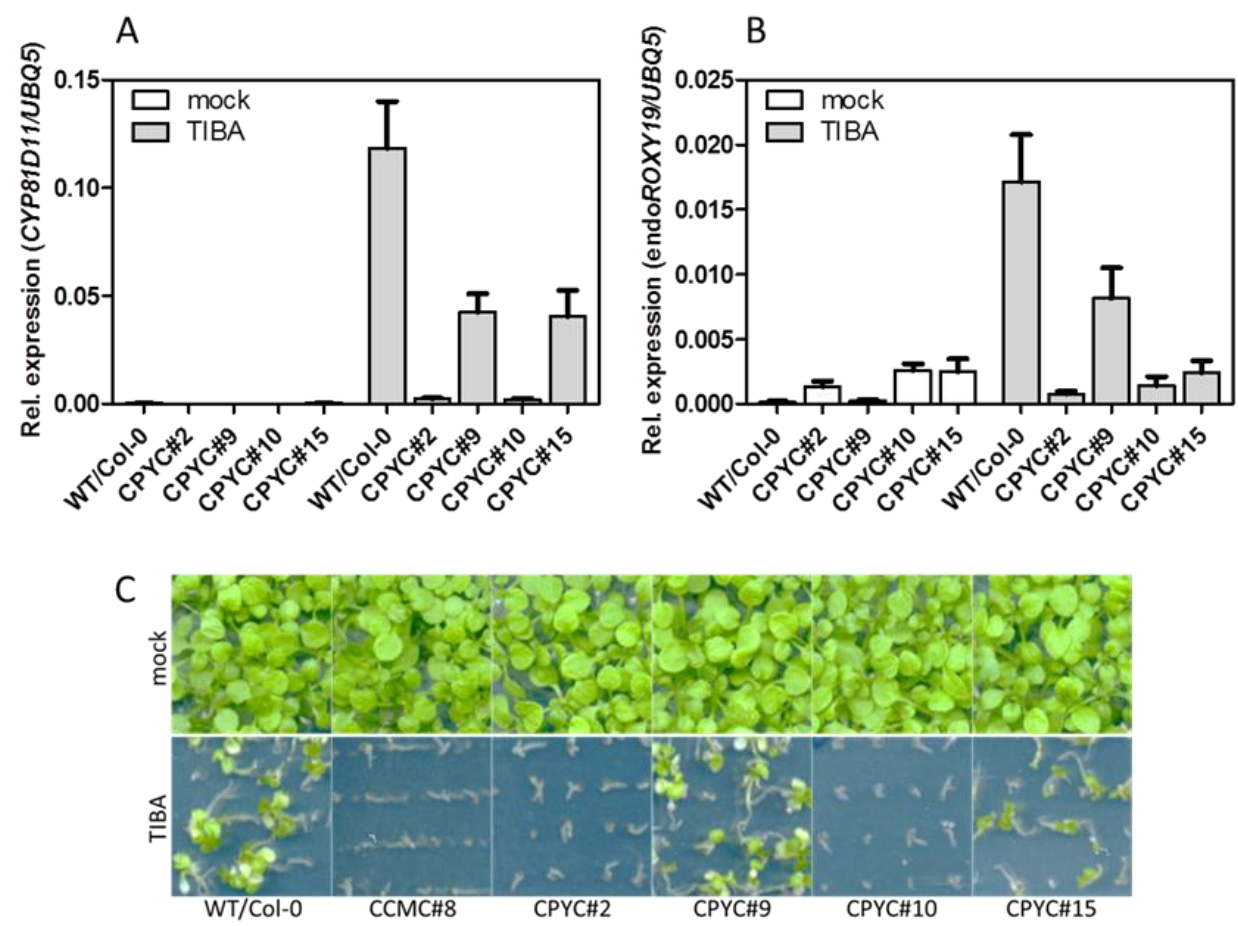

Figure 3.14 The 35S:HA-ROXY19CPYC transgenic plants repress TIBA-induced CYP81D11 and endogenous ROXY19 expression and are more sensitive to TIBA treatment. (A) and (B) qRT-PCR analysis of CYP81D11 and endogenous ROXY19 in WT Col-0 and 35S:HA-ROXY19CPYC transgenic plants. Arabidopsis seeds were germinated on soil and grown for four weeks, and subsequently sprayed with DMSO (mock) or 0.1mM TIBA. After $10 \mathrm{~h}$ treatment, plant leaves were harvested for RNA extraction and CDNA synthesis, CYP81D11 and endogenous ROXY19 transcripts were analyzed with qRT-PCR. The mean values ( $\pm S E$ ) from 4-5 independent replicates (one pot with one plant as one biological replicate) are shown. (C) The 35S:HA-ROXY19CPYC transgenic plants are more sensitive to TIBA. Arabidopsis seeds were germinated and grown on MS-plates containing DMSO (mock) or 50 MM TIBA for two weeks. 
To obtain a loss-of-function phenotype, we examined whether the roxy19 mutant is more resistant to xenobiotics. Considering potential gene redundancy, our lab had crossed roxy19DS with roxy18 (a T-DNA insertion line in Col-0 background) to generate a double mutant in which the two most homologous ROXYs are simultaneously mutated (AG Gatz). Although transgenic plants expressing ROXY19 are more sensitive to xenobiotic stress (Figure 3.11B and 3.13), no enhanced resistance in the roxy18 roxy19DS double mutant was observed as compared to Nossen after growth on TIBAcontaining plates (Figure 3.8G).

\subsubsection{ROXY19 is induced by xenobiotic stress}

Consistent with the occurrence of TGA-binding sites in its promoter, we found that the expression of ROXY19 was induced by TIBA in Col-0 plants (Figure 3.11A). In addition, the TIBA-induced endogenous ROXY19 was repressed in 35S:HA-ROXY19CCMC plants (lines CCMC\#8 and \#12) but not in 35S:HA-ROXY19SSMS plants (lines SSMS\#9 and \#18) (Figure 3.11A). Again, the 35S:HAROXY19CPYC transgenic plants were used to check the expression of endogenous ROXY19. The qRTPCR analysis revealed that endogenous ROXY19 was repressed in those three lines (CPYC lines\#2, 10 and \#15) (Figure 3.14B), that show strong ROXY19 protein expression (Figure 3.7B). This result indicates that, like detoxification genes, ROXY19 is also responsive to xenobiotic stress and can be repressed by ROXY19.

\subsubsection{Class II TGA factors are required for TIBA-induced ROXY19 expression}

We have shown that ROXY19 promoter activity was activated by class II TGA transcription factors (TGA2) in protoplasts (Figure 3.2A). We examined whether class II TGA factors are required for TIBAinduced ROXY19 expression. 30-days-old soil grown plants were treated with $100 \mu \mathrm{M}$ TIBA solution. qRT-PCR analysis showed that TIBA-induced ROXY19 expression is hampered in the tga256 mutant (Figure 3.15A). The induction of ROXY19 is recovered in transgenic plants expressing TGA5 (line 35S:TGA5\#1) or TGA5C ${ }^{186} \mathrm{~S}$ (line 35S:TGA5C ${ }^{186} \mathrm{S \# 12}$ ) in the tga256 mutant background (Figure 3.15A). It has been known that the tga256 mutant is more sensitive to TIBA (Fode et al., 2008). We found that both TGA5 and TGA5C ${ }^{186} \mathrm{~S}$ restore TIBA-sensitivity of tga256 to Col-0 level (Figure 3.15B). Under these conditions, the existence of the putative redox-active cysteine is not important.
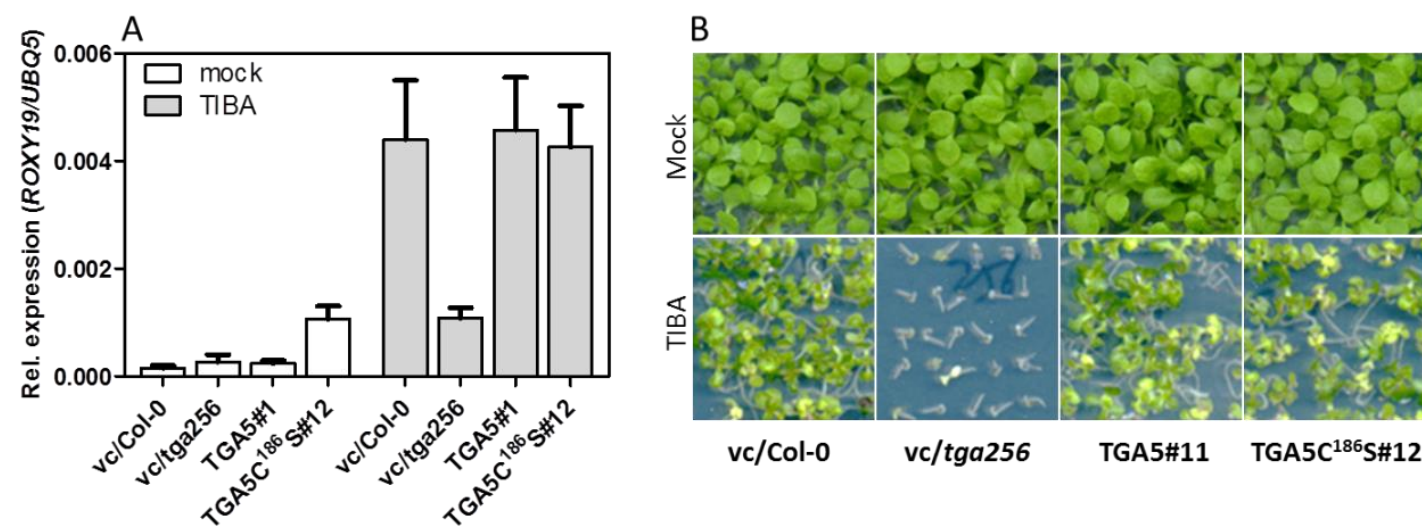

Figure 3.15 Characterization of tga256 mutant and complementation lines in response to xenobiotic treatment. (A) TIBA induces ROXY19 expression in a class II TGA-dependent way. Arabidopsis seeds were germinated on soil and grown for four weeks, and subsequently sprayed with DMSO (mock) or $0.1 \mathrm{mM}$ TIBA solution. After $10 \mathrm{~h}$ of treatment, plant leaves were harvested for RNA extraction and CDNA synthesis, ROXY19 transcripts were analyzed with qRT-PCR. The mean values $( \pm S E$ ) from 5 independent replicates (one soil pot with one plant as one biological replicate) are shown. (B) The tga256 mutant is more sensitive to xenobiotic TIBA. Arabidopsis seeds of were germinated and grown on MS-plates containing DMSO (mock) or 50 $\mu \mathrm{M}$ TIBA for two weeks. 


\subsection{ROXY19 facilitates susceptibility to necrotrophic fungus Botrytis cinerea}

Since defense hormone-induced PDF1.2 expression is repressed in ROXY19 overexpressing plants (Ndamukong et al., 2007 and Supplemental Figure S4), we examined whether these plants are more susceptible to infections with necrotrophic pathogens. As expected, the 35S:HA-ROXY19CCMC plants (lines CCMC\#8 and \#12) were more susceptible to Botrytis cinerea with larger lesion size than Col-0 and the 35S:HA-ROXY19SSMS plants (lines SSMS\#9 and \#18) (Figure 3.16A). Consistent with these results, gene expression analysis revealed that pathogen triggered PDF1.2 expression (Figure 3.16B) and also JA-induced PDF1.2 expression (Supplemental Figure S4) is repressed in 35S:HAROXY19CCMC plants but not in 35S:HA-ROXY19SSMS plants. The results confirm that the active site is important for ROXY19 function. Botrytis cinerea-induced PDF1.2 expression was also strongly repressed in lines CPYC\#2 and CPYC\#10 (Figure 3.16C). Since line CPYC\#9 does not express the protein, PDF1.2 can be induced. As observed before (Figures 3.7), line CPYC\#15, which expresses similar amounts of HA-ROXY19 as lines \#2 and \#10, does not repress PDF1.2. The same pattern was observed in JA-induced plants (Supplemental Figure S5). Still, the consistent repressive activity observed in lines \#2 and \#10 supports the notion that the CCMC motif can be exchanged by the CPYC active site. Thus, the specific function of the CCMC sequence has remained elusive.

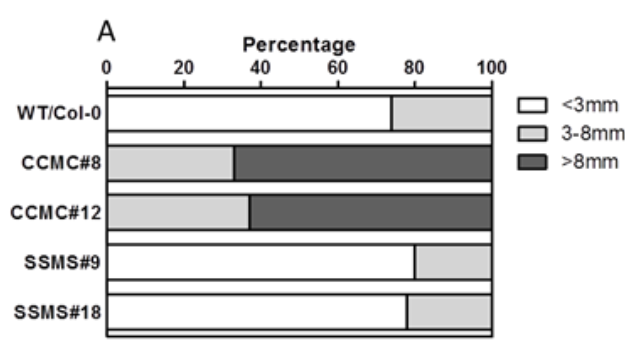

Figure 3.16 Symptom development and PDF1.2 expression in Col-0 and ROXY19 transgenic plants after infection with Botrytis cinerea. (A) Lesion size on Col-0 and ROXY19 transgenic plants at three days post infection with $B$. cinerea. Leaves of four-week-old soil grown plants were drop-inoculated with a $B$. cinerea spore solution $\left(5 \times 10^{4}\right.$ spores per $\mathrm{ml}$ ) or quarter-strength potato dextrose broth (mock). The diameters of at least 30 lesions per experiment were measured and grouped according to their size into the three indicated classes. (B) qRT-PCR analysis of PDF1.2 expression in Col-0 and 35S:HA-ROXY19CCMC transgenic plants after $B$. cinerea infection. Leaf samples from Figure 3.16A were harvested for RNA extraction. (C) qRT-PCR analysis of PDF1.2 expression in Col-0 and 35S:HA-ROXY19CPYC transgenic plants after $B$. cinerea infection. Leaves of four-week-old soil grown plants were drop-inoculated with a $B$. cinerea spore solution $\left(5 \times 10^{4}\right.$ spores per $\left.\mathrm{ml}\right)$ or quarter-strength potato dextrose broth (mock). Three days after infection, leaf samples were harvested for RNA extraction. The mean values $( \pm S E$ ) from four independent replicates (samples from 3-4 plants as one biological replicate) of infected samples are shown. One replicate of uninfected samples are shown.

\subsection{Development of a chemical inducible ROXY19 expression transgenic line}

Our results with the stable transgenic plants show that ROXY19 represses pathogen and JA-induced PDF1.2 expression (Figure 3.16 and Supplemental Figure S4). In order to investigate whether the repression can directly occur after induction of ROXY19 expression, we used a $\beta$-estradiol-inducible vector (Curtis and Grossniklaus, 2003) to generate Arabidopsis transgenic plants expressing ROXY19. The resulting lines were called XVE lines (Figure 3.17). In the presence of the inducing chemical, $\beta$ estradiol, line XVE\#9 showed ROXY19 protein expression to a similar level of a previous produced constitutive expression line (Figure 3.17B). After ACC/JA-treatment of MS-grown plants, both WT and 
XVE\#9 plants showed strong PDF1.2 induction. Although the induction already become compromised in Col-0 in the presence of $\beta$-estradiol, the PDF1.2 expression was more repressed in XVE\#9 than in Col-0 (Figure 3.17C). The induction was totally abolished in the previously generated 35S:HA-ROXY19CCMC\#8 plants. The result suggests that an inducible ROXY19 expression can also repress ET/JA-induced PDF1.2.

A

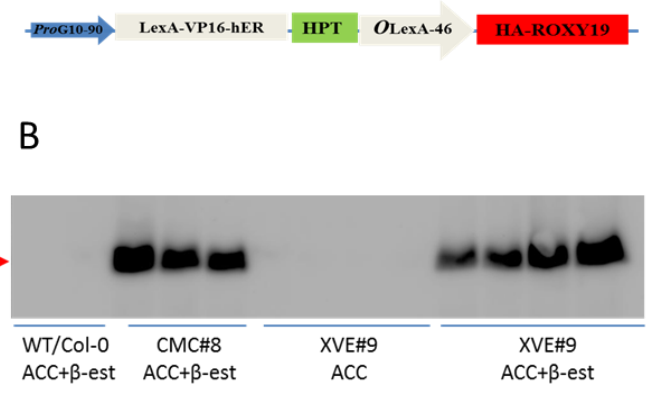

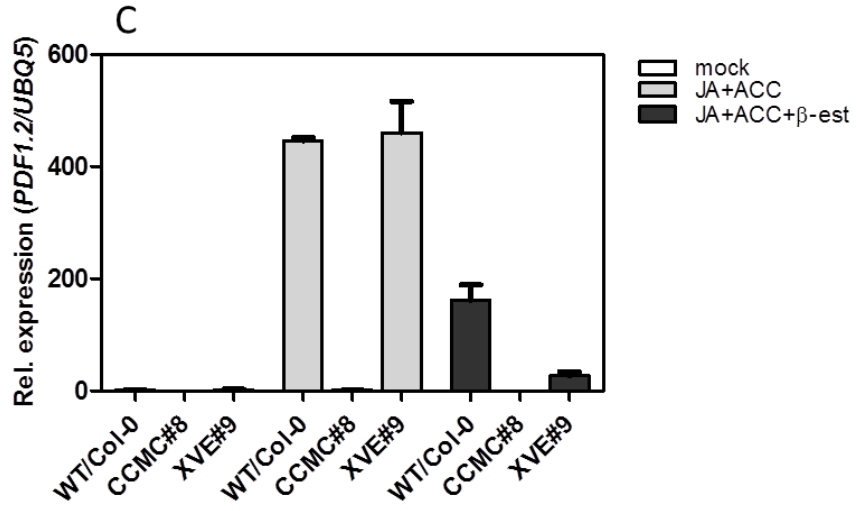

Figure 3.17 Development and characterization of transgenic plants containing ROXY19 under the control of a chemically inducible promoter. (A) Schematic diagram of the inducible expression construct. Only the core region between T-DNA left and right border is shown. A synthetic promoter, ProG10-90, controls the expression of the transactivator, LeXA-VP16-hER (XVE), which encodes a chimeric transcription factor consisting of the DNA-binding domain of LexA, the transcription activation domain of VP16 and the ligand-binding domain of the human estrogen receptor; upon induction, XVE binds to the LexA operators upstream of a core promoter (OLeXA-46) and induces the expression of HA-tagged ROXY19. (B) Western blot analysis of the ROXY19 expression. Protein extracts as indicated were prepared from the same plant samples (2 to 4 replicates) used for RNA preparation in Figure 3.17C. HA-ROXY19 protein was detected with anti-HA. Red arrow denotes ROXY19 specific bands. (C) qRT-PCR analysis of PDF1.2 expression in chemically induced ROXY19 plants after hormone and chemical treatment. Col-0, 35S:HA-ROXY19CCMC\#8 and XVE\#9 transgenic plants were grown vertically on MS-plates for 12 days and subsequently transferred to MS-plates containing 0 or $10 \mu \mathrm{M} \beta$-est and supplemented with $0.01 \%$ ethanol (mock) or $5 \mu \mathrm{M}$ MeJA in $0.01 \%$ ethanol $(\mathrm{JA})$ and $500 \mu \mathrm{M} \mathrm{ACC}$ as indicated. After $48 \mathrm{~h}$ of treatment, approximately 50 seedlings were harvested for RNA and protein (B) extraction. The mean values ( \pm SE) from four independent replicates (one treatment with 50 seedlings as one replicate) are shown.

\subsection{Inhibiting DNA methylation cannot recover PDF1.2 expression in ROXY19 transgenic plants}

Since epigenetic regulation such as DNA methylation interferes with gene expression, it is interesting to known whether the promoter of PDF1.2 or upstream genes become hyper-methylated in ROXY19 overexpression plants. We grew Arabidopsis plants on MS-plates containing DNA methylation inhibitor 5-azacytidine (5-azaC) to erase gene methylation. However, after ACC/JA-induction the expression of PDF1.2 was still repressed in 5-azaC treated plants (Figure 3.18A).

Although these results suggest that ROXY19 does not repress PDF1.2 expression through DNA hypermethylation, we set out to check whether SA may manipulate DNA methylation of ET/JA-mediated genes to repress their expression. Both plants grown in the absence or presence of 5-azaC showed strong PDF1.2 expression after induction, though plants grown with inhibitor had an alleviated induction, the induction was repressed by SA (Figure 3.18B). As control the expression of a DNA 
methylation marker gene, FWA, was dramatically induced in all plants grown with 5-azaC (Figure 3.18C). These results reveal that DNA methylation does not play a major role in SA-mediated repression of ET/JA-induced PDF1.2 expression.
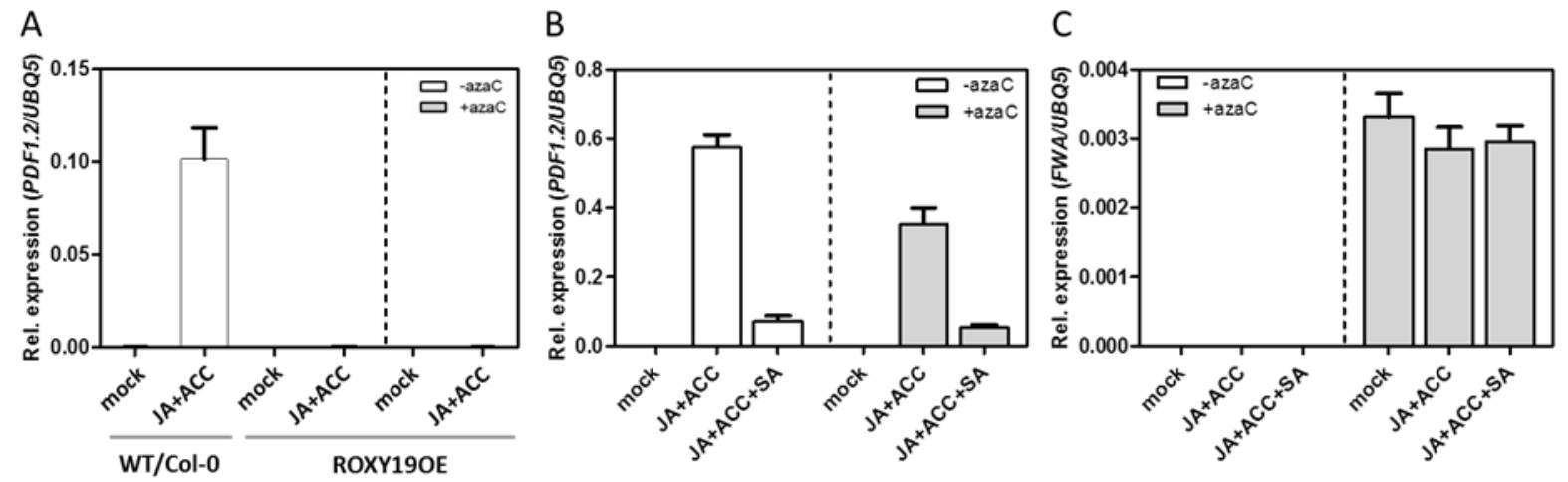

Figure 3.18 DNA methylation does not play a major role in regulating PDF1.2 expression. (A) Inhibiting of DNA methylation cannot recover PDF1.2 expression in ROXY19 gain-of-function plants. ROXY19 transgenic line (ROXY19OE) was germinated and grown vertically on MS-plates with or without $25 \mu \mathrm{M} 5$-azaC (5Azacytidine) for 12 days and subsequently transferred to MS-plates containing 0 or $25 \mu \mathrm{M} 5$-azaC and supplemented with $0.01 \%$ ethanol (mock) or $5 \mu \mathrm{M}$ MeJA in $0.01 \%$ ethanol (JA) and $500 \mu \mathrm{M}$ ACC as indicated. As control, Col-0 plants was germinated and grown vertically on MS-plates for 12 days and subsequently transferred to MS-plates containing 0 or $25 \mu \mathrm{M}$-azaC and supplemented with $0.01 \%$ ethanol (mock) or $5 \mu \mathrm{M}$ MeJA in $0.01 \%$ ethanol (JA) and $500 \mu \mathrm{M} \mathrm{ACC}$ as indicated. After $48 \mathrm{hr}$ of treatment, approximately 50 seedlings were harvested for RNA extraction. The mean values ( $\pm \mathrm{SE}$ ) from four independent replicates (one treatment with 50 seedlings as one replicate) are shown. (B) qRT-PCR analysis of PDF1.2 expression in hormone treated Col-0 plants grown on MS-plates with or without 5-azaC. Col-0 seeds were germinated and grown vertically on MS-plates with or without $25 \mu \mathrm{M} 5$-azaC for 12 days, and then transferred to MS-plates supplemented with $0.01 \%$ ethanol (mock) or $5 \mu \mathrm{M}$ MeJA in $0.01 \%$ ethanol (JA) plus $500 \mu \mathrm{M} \mathrm{ACC}$ or $200 \mu \mathrm{M} \mathrm{SA}$ as indicated. After $24 \mathrm{~h}$ of treatment, approximately 50 seedlings were harvested for RNA extraction. The mean values $( \pm S E)$ from five independent replicates (one treatment with 50 seedlings as one biological replicate) are shown. (C) qRT-PCR analysis of FWA expression in hormone treated Col-0 plants grown on MS-plates with or without 5-azaC. RNA samples were the same used for analysis in Figure 3.18B.

\subsection{Characterization of the roles of class II TGA factors in Arabidopsis}

\subsubsection{The cysteine of class II TGAs is not important for regulating PDF1.2 expression}

Since $S A$ represses the ET/JA-induced expression of defense genes and since these genes depend on class II TGA transcription factors, it has been hypothesized that $S A$ induces a regulatory protein that interferes with class II TGA activity. A candidate protein is ROXY19, as its expression is induced by SA and as ROXY19 protein interacts with TGA factors. Moreover, the ET/JA-signaling pathway is suppressed in ROXY19 overexpressing plants. Since the active site of ROXY19 is important for efficient repression of target genes, it is possible that the potential oxidoreductase activity of ROXY19 interferes with TGA function by controlling its redox state.

All three members of class II TGA factors contain only one conserved cysteine (Cys186). In order to investigate whether the cysteine is important for class II TGA factors in regulating PDF1.2 expression, the cysteine residue was mutated to serine (Ser/S). We generated transgenic Arabidopsis plants 
expressing TGA5 and TGA5C ${ }^{186} \mathrm{~S}$ under the control of CaMV35S promoter in the tga256 triple mutant. Transgenic plants showed high expression of TGA5 (lines TGA5\#7, \#11 and \#21) and TGA5C ${ }^{186} \mathrm{~S}$ (lines $\# 12$, \#14 and \#20) as shown by Western blot analysis (Figure 3.19A). Transgenic plants grown on MSplates were used for the hormone crosstalk assays. As reported previously, ACC/JA-treatment strongly induced expression of the marker gene PDF1.2 and the induction was repressed in the presence of SA (Zander et al., 2010). In the tga256 triple mutant, induction ACC/JA induction was abolished and SA slightly induced PDF1.2 expression in a previous report (Zander et al., 2010). Both 35S:TGA5 and 35S:TGA5C ${ }^{186} S$ transgenic plants exhibited stronger ACC/JA-induced PDF1.2 expression as compared to Col-0 plants. Moreover the induction was repressed by addition of SA (Figure 3.19B). This result suggests that the conserved Cys residue is not important for TGA5 function in terms of regulating $A C C / J A$-induced and SA-repressed PDF1.2 expression.

The previously generated Arabidopsis transgenic plants expressing TGA2 (lines TGA2\#7F and \#13A) and TGA2C $C^{186} \mathrm{~S}$ (lines TGA2C ${ }^{186} \mathrm{~S} \# 4 \mathrm{C}$ and \#6D) under the CaMV35S promoter (AG Gatz) were used to test the importance of Cys residue of TGA2. Western blot analysis revealed these transgenic plants expressed protein level similar to Col-0, but much less than the 35S:TGA5 transgenic plants (Figure 3.19C). Hormone crosstalk assays showed that both WT and TGA2C ${ }^{186} S$ restore ACC/JA-induced PDF1.2 expression. However, the negative effect of SA was not as strong as the observed in WT and 35S:TGA5 plants (Figure 3.19D). This result indicates that the conserved Cys residue is not important for TGA2 function in regulating PDF1.2 expression.
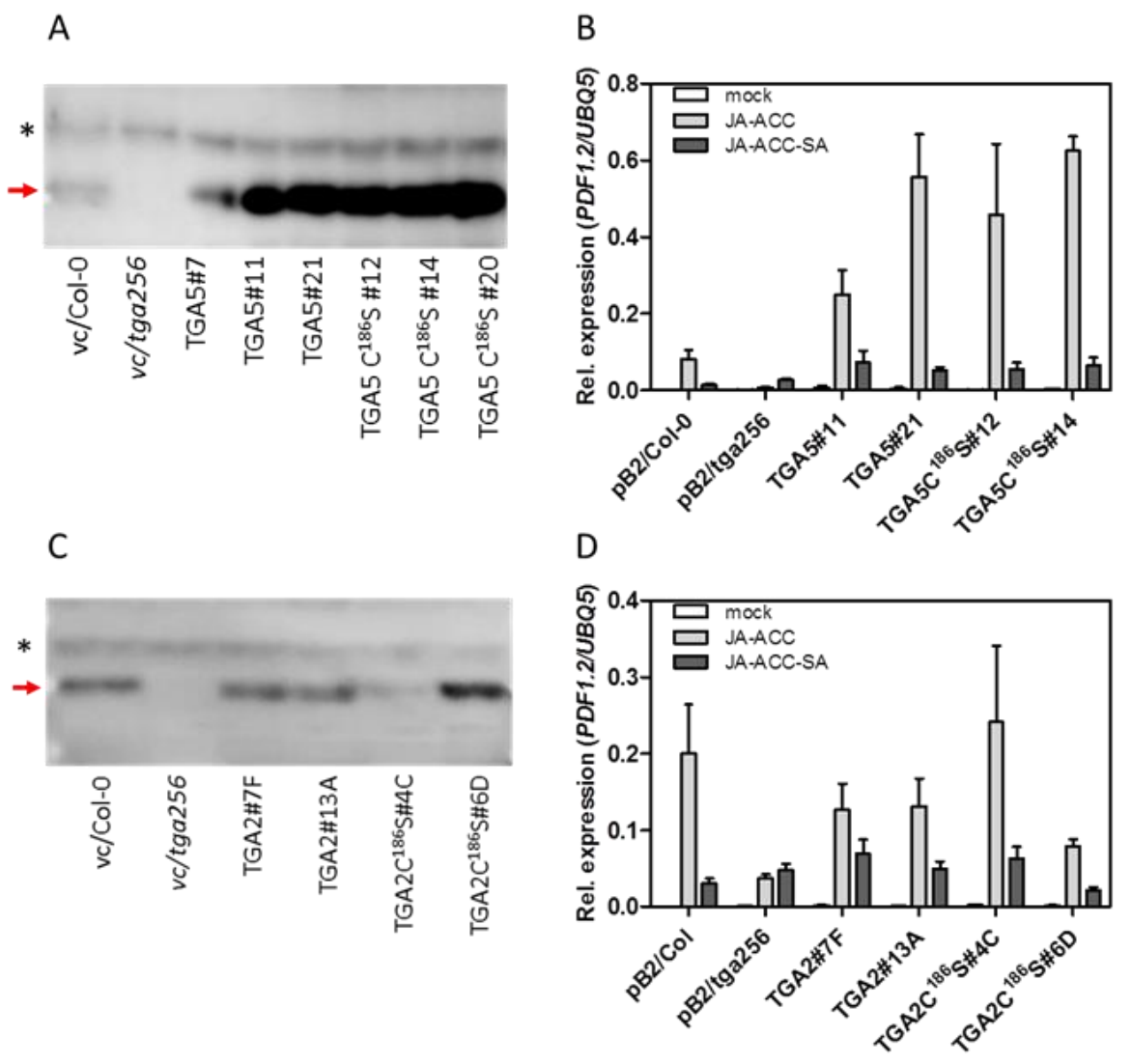

Figure 3.19 Complementation of tga256 triple mutant plant with WT and cys mutant TGA factors. (A) Western blot analysis of 35S:TGA5 and 35S:TGA5C ${ }^{186} S$ transgenic using antibody against TGA2 and TGA5. (B) Quantitative real-time RT-PCR (qRT-PCR) analysis of PDF1.2 expression in complementation plants after hormone treatment. (C) Western blot analysis of 35S:TGA2 and 35S:TGA2 $C^{186} S$ transgenic plants using 
(Figure 3.19 continued) antibody against TGA2 and TGA5. (D) qRT-PCR analysis of PDF1.2 expression in transgenic plants after hormone treatment. In Figure $(A)$ and $(C)$, asterisk and arrow indicate unspecific bands which serve as loading control and specific bands, respectively. In Figure (B) and (D), Arabidopsis seeds of each genotype were germinated on MS-plates and grown vertically for 12 days, and subsequently transferred to MS-plates supplemented with $0.01 \%$ ethanol (mock) or $5 \mu \mathrm{M}$ MeJA in $0.01 \%$ ethanol (JA) and $500 \mu \mathrm{M}$ ACC, or $200 \mu \mathrm{M}$ SA as indicated. After $48 \mathrm{~h}$ of treatment, approximately 50 seedlings were harvested for RNA or protein extraction. The mean values $( \pm S E)$ from four independent replicates (one MS-plate with 50 seedlings as one biological replicate) are shown.

\subsubsection{Defense hormone treatment does not influence protein stability of class II TGAs}

Pontier et al. (2002) reported that certain TGA proteins (TGA1 and TGA3) were post-translationally regulated by proteasome-mediated proteolysis. As the transgenic TGA5 complemented the tga256 mutant (Figure 3.19B and D), we continued to examine whether TGA5 protein level is regulated by ACC/JA and SA treatments. Protein was extracted from hormone treated samples used in Figure 3.19B. However, our result showed that both endogenous TGA5 (and TGA2) protein in Col-0 plants and 35S:TGA5 transgenic plants were quite stable in response to different hormone treatments (Figure 3.20).

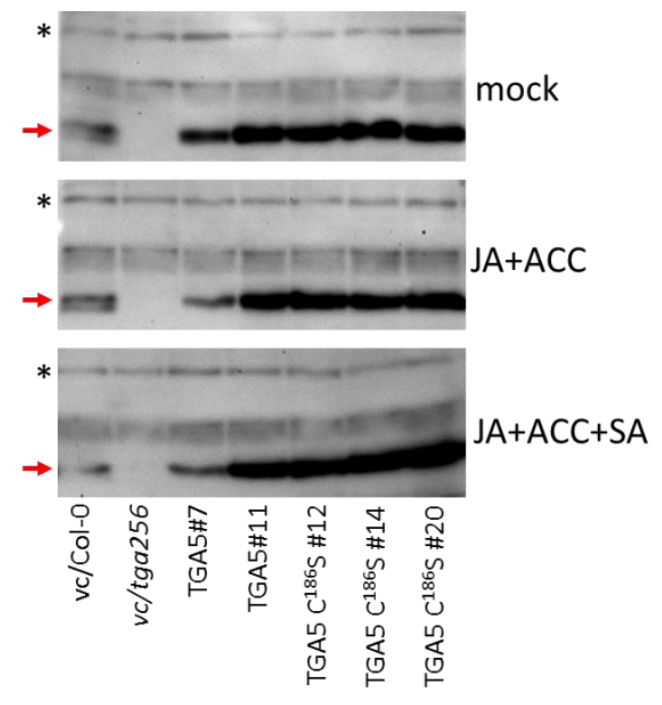

Figure 3.20 Protein stability of TGA5 is not regulated by defense hormones. Detection of TGA5 protein using antibody against TGA2 and TGA5 after hormone treatments in transgenic plants. Protein was extracted from plant samples used for RNA extraction in Figure 3.19B. Asterisk and arrow indicate unspecific bands, which serve as loading control, and specific bands, respectively.

\subsubsection{Class I TGAs repress the ET/JA-induced PDF1.2 expression in the absence of class II TGAs}

The Arabidopsis genome encodes 10 TGA genes which are divided into five classes. Studies have shown that individual TGA proteins vary in their ability to regulate target gene expression. For instance, it has been shown that class I TGA1 and class II TGA5 have opposite effects on the octopine synthase (ocs) element (Foley and Singh, 2004). Like the as-1 element, the ocs element - originally identified in Agrobacterium T-DNA gene - is recognized by TGA factors. Genetic analysis revealed that class II TGA factors are required for both ET/JA-induced and SA-repressed PDF1.2 gene expression (Zander et al., 2010). Whether other TGA factors are involved in these hormone responses is not characterized. However, our hormone crosstalk assay showed that ACC/JA-induced and SA-repressed PDF1.2 expression was not affected in the tga14 double mutant (Figure 3.21A).

The tga12456 pentuple mutant was generated by crossing the tga256 with the tga14 mutant and a homozygous line was selected by genotyping PCR in F2 generation (AG Gatz). Next we performed hormone crosstalk assays using Arabidopsis seedlings grown on MS-plates. We found that the ACC/JA-induced PDF1.2 expression, which was abolished in tga256, was recovered in the tga12456 
mutant (Figure 3.21A and C). This means that class I TGA factors can function as negative transcription factors and that the function of class II TGA factors is to prevent their access to the promoter. Moreover, induction in tga12456 mutant was not repressed by SA (Figure 3.21A), this result confirms pervious finding that SA-mediated repression is class II TGA factors dependent (LeonReyes et al., 2010; Zander et al., 2010).

We confirmed our findings that class I TGA factors are repressors of PDF1.2 gene expression in the absence of class II TGA factors in soil grown plants. However, recovered PDF1.2 expression in tga12456 mutant was still significantly repressed by SA (Figure 3.21C) indicating that under these conditions hitherto not described class II TGA-independent negative effect of SA is operational. This discrepancy observed among MS-plates grown seedlings and soil grown mature plants requires further clarification. Nevertheless, since both Col-0 and tga14 double mutant showed a similar induction of PDF1.2 expression, it seems like that class I TGA factors do not interfere class II TGA factors activated PDF1.2 expression in WT Col-0 plants. Zander et al. (2014) showed that class II TGA factors regulate the ET/JA-signaling pathway via directly binding to ORA59 promoter and activating ORA59 expression. Thus it is of interest to know whether JA/ACC-induced ORA59 are recovered in the tga12456 plants. However, in this experiment, the expression of ORA59 was inconsistent (Figure $3.21 \mathrm{~B}$ and $\mathrm{D})$.

The expression of JA-responsive CYP81D11 is also class II TGA factors dependent (Köster et al., 2012). Thus we examined the induction of CYP81D11 in the tga12456 plants. However, the JA-induced CYP81D11 expression was not recovered in tga12456 (Figure 3.21E).

It has been known that tga256 mutant plants are more sensitive to SA (Fode et al., 2008). As the ET/JA-induced PDF1.2 was restored in tga12456 plants, we further tested whether SA sensitivity is restored in tga12456; however like tga256, tga12456 plants grown on SA-containing plates are more sensitive and become chlorosis as compared to WT Col-0 (Figure 3.21F). These results suggest not all alternations observed in tga256 mutant can be suppressed by tga14 mutant. 

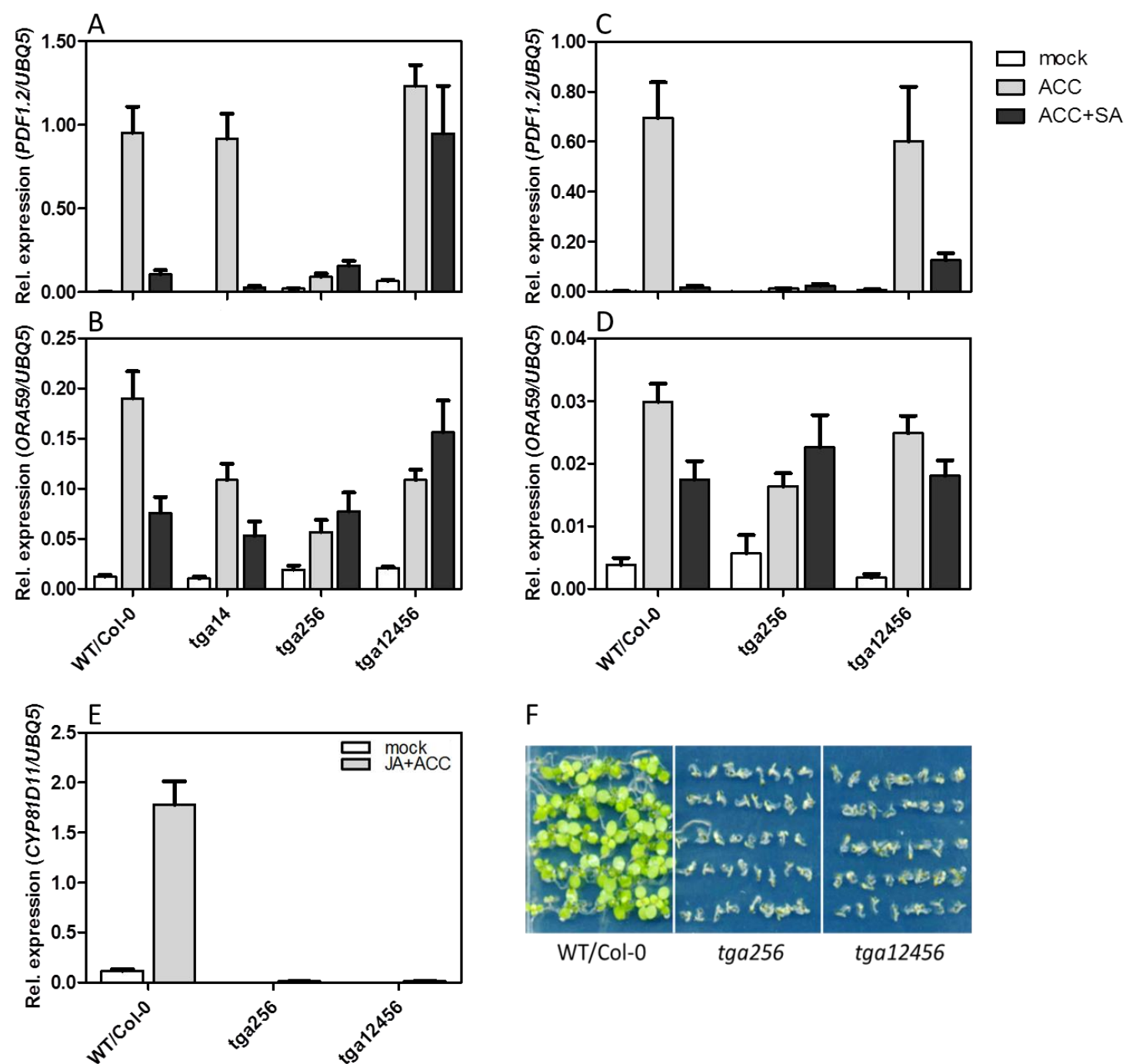

$\mathrm{F}$

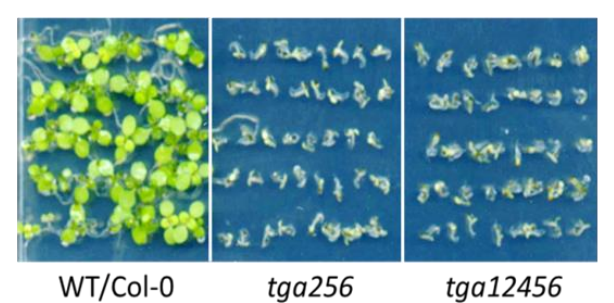

Figure 3.21 Class I TGA factors repress the ET/JA-induced PDF1.2 expression in the absence of class II TGA factors. (A) and (B) qRT-PCR analysis of PDF1.2 and ORA59 expression in WT/Col-0, tga14, tga256 and tga12456 grown on MS- plates after hormone treatment. Col-0, tga14, tga256, and tga12456 were grown vertically on MS-plates for 12 days and subsequently transferred to plates supplemented with $0.01 \%$

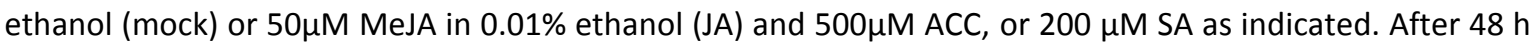
of treatment, approximately 50 seedlings were harvested for RNA extraction. (C) and (D) qRT-PCR analysis of PDF1.2 and ORA59 expression in WT/Col-0, tga14, tga256 and tga12456 mutant grown on soil after hormone treatment. Col-0, tga14, tga256, and tga12456 were grown on soil (one plant/ pot) for four week, and then sprayed with $1 \mathrm{mM}$ ACC or $1 \mathrm{mM} \mathrm{SA}$. After $24 \mathrm{~h}$ of treatment, approximately 50 seedlings were harvested for RNA extraction. (E) qRT-PCR analysis of CYP81D11 expression in WT/Col-0, tga256 and tga12456 mutant after hormone treatment. Col-0, tga256, and tga12456 were grown vertically on MSplates for 12 days and subsequently transferred to MS-plates supplemented with $0.01 \%$ ethanol (mock) or $50 \mu \mathrm{M}$ MeJA in $0.01 \%$ ethanol (JA) and $500 \mu \mathrm{M}$ ACC as indicated. After $48 \mathrm{~h}$ of treatment, approximately 50 seedlings were harvested for RNA extraction. (F) Growth phenotype of 10-day-old WT/Col-0, tga256 and tga12456 seedlings on MS-plates containing 50 $\mathrm{M}$ SA. Like tga256, tga12456 quintuple mutant was more sensitive to SA and cannot grow over the cotyledon stage. For qRT-PCR analysis, the mean values ( \pm SE) from four independent replicates are shown. 


\section{Discussion}

\subsection{ROXY19-mediated repression requires a functional active site}

ROXY19 belongs to the 21-membered family of ROXY genes. As observed also for its closest homolog, ROXY18, ROXY19 expression is induced upon infection by necrotrophic and biotrophic pathogens. Whereas roxy18 mutant plants are more resistant to the necrotrophic pathogen $B$. cinerea (La Camera et al., 2011), no phenotype has yet been observed for roxy19 plants. However, ectopically expressed ROXY19 represses the ORA59 promoter after ET treatment (Zander et al., 2012) and its own promoter after SA treatment (Herrera-Vásquez et al., 2014). In the first part of the thesis, experiments addressing the mechanism of gene repression were addressed.

To this aim, we chose the ROXY19 promoter as a target promoter for the repressive effect of ROXY19. TGA factors and their binding sites are important for the expression of this promoter after SAmediated activation of the transcriptional co-activator of TGA factors, NPR1. Moreover, their binding sites and TGA factors are important for its activity in protoplasts (Figure 3.1C and 3.2A). In contrast, $J A$ induction of the promoter, which is driven by MYC2 binding to the G-box, does not require TGA factors (Köster et al., 2012). Still, TGA factors, which are constitutively bound to the promoter as revealed by ChIP experiments (Herrera-Vásquez et al., 2014) are required for the repressive effect of ROXY19. Moreover, TGA factors are required for the repression in transient assays (Figure 3.2A). Figure 4.1 displays a sketch of the ROXY19 promoter under different states.

Resting state

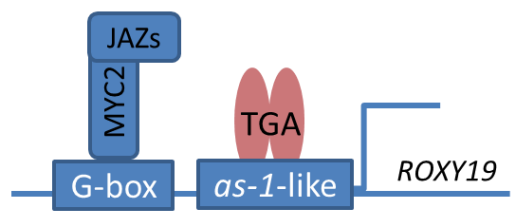

SA induction

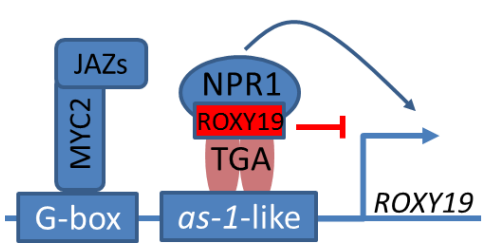

$\mathrm{JA}$ induction

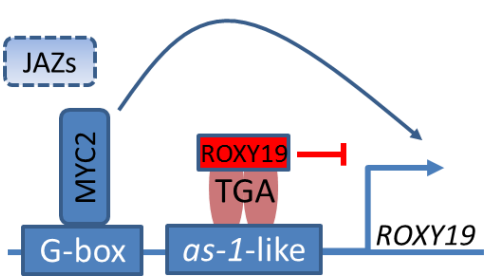

TIBA induction

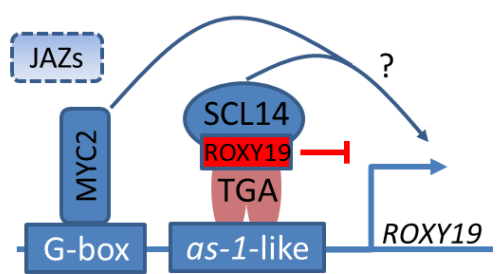

Figure 4.1 The expression of ROXY19 under different conditions. 1) In the resting state, both TGAs and MYC2 bind to their target sites, the as-1-like element and the G-box in the ROXY19 promoter, respectively, however no transcription activator is available for TGAs and transcription activity of MYC2 is repressed by JAZ proteins. 2) Upon SA induction, activated NPR1 interacts with TGAs to induce ROXY19 transcription. 3) Upon JA induction, JAZ proteins are degraded and MYC2 is released to activate ROXY19 expression. 4) Based on current knowledge, we hypotheses that upon TIBA induction, SCL14 interacts with TGAs. In addition, TIBA-triggered JA signaling leads to JAZs degradation and releasing of MYC2. Like TIBA-induced CYP81D11 expression, both TGAs and MYC2 are required for maximal ROXY19 expression. In all induction circumstances, ectopically expressed or induced ROXY19 binds to TGAs to repress its own promoter activity, possibly through recruiting the transcriptional co-repressor TPL. TGAs are required for both induction and repression of ROXY19 promoter after SA and TIBA induction and in protoplasts. Experimental evidences are missing that MYC2 and SCL14 are required to activating ROXY19 expression upon TIBA 
In order to get closer to the mechanism of repression, we performed functional assays both in transiently transformed protoplasts and in stable transgenic plants. Table 4.1 summarizes our observations.

Table 4.1 Factors that regulate ROXY19-mediated repression in protoplasts and plants

\begin{tabular}{|l|l|l|}
\hline Factor Method & Transiently transformed protoplasts & Stable transgenic plants \\
\hline Class II TGAs & Yes & Yes \\
\hline Cys of class II TGAs & No & No \\
\hline TPL (ALWA) & Yes & Yes \\
\hline GSH (G111A) & Yes & Yes \\
\hline Active site (CCMC) & No & Yes \\
\hline
\end{tabular}

As summarized in the table, so fa we found that: class II TGAs, co-repressor TPL and GSH binding are essential for ROXY19 repressive effect in both stable transgenic plants and transiently transformed protoplasts; the active site is required in transgenic plants but not in protoplasts; the conserved Cys of class TGAs seems to be irrelevant to ROXY19 function.

Our preliminary data imply that ROXY19 may recruit TPL, a transcriptional co-repressor. This assumption is based on the observation that the C-terminal ALWL motif, which is critical for ROXY19mediated repression of the ORA59 promoter (Zander et al., 2012) and of the ROXY19 promoter in transient assays (Figure 3.1 and 3.4), is responsible for the interaction with TPL in yeast two hybrid assays (Dr. Joachim Uhrig, personal communication). Expression of the $\mathrm{N}$-terminal domain of TPL, which interacts with the ALWL motif, intercepts the repressive capacity of ROXY19 (Figure 3.4B), indicating that the $\mathrm{N}$-terminal domain blocks the access of the endogenous protein to the ALWL motif. Conclusive evidence will include the loss-of-function mutant in which the activity of the fivemembered TPL gene family is compromised. Wang et al. (2013) showed that TPL-repressed circadian period was significantly lengthened only when all five members were depleted by using artificial microRNA (amiRNA). Since the tpl-2 tpr1 tpr3 tpr4 quadruple and the tpl-2 tpr1 tpr2 tpr3 tpr4 pentuple loss-of-function mutants are available (Krogan et al., 2012), we can investigate the effect ROXY19-mediated repression in these mutants now.

The importance of the ALWL motif has been observed by Li et al. (2009), who have shown that only ROXYs with an ALWL motif (ROXY1, ROXY2, ROXY3, ROXY4, ROXY5, ROXY18 and ROXY19) but not ROXYs lacking the ALWL motif (ROXY6, ROXY7, ROXY8, ROXY9 and ROXY20) can complement the roxy1 flower phenotype when expressed under the ROXY1 promoter. Thus, recruitment of TPL to TGA-regulated target promoters might be a conserved mechanism of those ROXYs that contain such a motif.

Another important feature of ROXYs is their assumed function to bind glutathione. Mutation of a glycine residue, which is conserved in all glutaredoxins, into an alanine residue is presumed to interfere with glutathione binding (Xing and Zachgo, 2008). This mutation interferes with the repressive capacity of ROXY19 both in transient assays (Figure 3.3A) and in transgenic plants (Zander et al., 2012) and with ROXY1 function in complementation experiments (Xing and Zachgo, 2008). However, the protein still interacts with TGA transcription factors. It is thus hypothesized that ROXY19 requires glutathione to repress gene expression. Therefore, we performed transient assays in protoplasts derived from pad2-1 and gr1 mutant plants. The pad2-1 mutant with a mutation in the Y-GLUTAMYLCYSTEINE SYNTHETASE 1 (GSH1) gene contains only about $22 \%$ of wild-type amounts of GSH (Parisy et al., 2007). Glutathione is maintained in the reduced state by glutathione reductase 
(GR). In Arabidopsis gr1 mutant, the amounts of oxidized glutathione (GSSG) increase approximately fourfold, while the amounts of reduced form (GSH) are not affected (Marty et al., 2009). However, repression was still observed pad2-1 and gr1 mutant protoplasts (Supplemental Figure 1). Since the residual glutathione levels might still be sufficient, it has remained an open question whether glutathione is required for the repressive activity of ROXY19. Evidence supporting direct binding of ROXYs to GSH is lacking.

Our transient expression assays in protoplasts showed that the active site is dispensable for ROXY19 function, while glutathione binding might be important (Figure 3.3A). However, qRT-PCR of selected genes and transcriptome analysis of stable transgenic plants expressing either ROXY19CCMC or ROXY19SSMS revealed that the active site is indeed important for the repression of target genes. However, the CCMC motif can be replaced by the CPYC motif (Figure 3.7), which still leaves the question open why the CCMC motif is so conserved. If we assume that the active site is important for the function of the protein as an oxidoreductase, we have to postulate that proteins at the promoter have to be redox-modified to allow the establishment of the repressive complex. These might be constitutively modified in protoplasts thus rendering the oxidoreductase activity of ROXY19 dispensable. Alternatively, it might well be that the high amounts of ROXY19 in the protoplast system can render the repressive complex independent of another redox-modulated yet unknown factor. Last, it has to be considered that the promoter is located in a plasmid in transiently transformed protoplasts, whereas it is integrated in the chromatin when we analyze ROXY function in stably transformed plants. It might well be that chromatin-associated events that depend on the active site of ROXYs have to be initiated. For ROXY1, it has been shown that the first but not the second cysteine of the active site is crucial for proper function of ROXY1 during petal development (Xing et al., 2005). And the predicted GSH binding site is important for ROXY1 function during anther development (Xing and Zachgo, 2008). Although, the conserved Cys340 is critical for PAN activity in flower development (Li et al., 2009), no evidence is available that PAN is redox modified by ROXY1. Alternatively, ROXYs might act as $\mathrm{Fe} / \mathrm{S}$-containing scaffold proteins that recruit TPL to target promoters. Biochemical analysis of ROXY19 in vitro is required and may provide new information to guide characterization in plants.

Our understanding of the repressive events in transgenic 35S:HA-ROXY19 plants is still very limited. Induction of elevated ROXY19 protein levels under the control of the $\beta$-estradiol-inducible promoter led to the repression of target promoters after 48 hours (Figure 3.17). However, we frequently observed that repression was not yet established in the first generation after transformation. In addition transgenic plants that express similar amounts of ROXY19 protein can be very different with respect to their capacity to repress target promoters (Figure 3.6 and 3.7). Therefore, there are still unknown parameters which influence the repression process. Once, repression is established, it is very stable. Therefore, we have started to investigate whether epigenetic effects might be involved in the establishment of the repressed state. However, growth of 35S:HA-ROXY19 plants on medium preventing methylation of the DNA did not relieve the repression (Figure 3.18A). Since it was found during the course of this thesis that TPL and therefore recruitment of histone deacetylase might be involved, it remains to be shown whether inhibitors of histone acetylation like Trichostatin A (TSA) would interfere with repression. For instance, in the presence of the TSA, TPL-mediated repression of the core clock genes was diminished in protoplasts (Wang et al., 2013). 


\subsection{ROXY19 suppresses the plant detoxification pathway}

As outlined in the introduction, class II TGA factors play a role in at least three distinct processes: systemic acquired resistance (SAR), activation of the ET/JA pathway and activation of the detoxification pathway upon chemical stress. Analysis of the transcriptome of unstressed plants ectopically expressing ROXY19 unraveled that genes of the detoxification pathway are repressed, an observation that was confirmed by qRT-PCR of selected genes from TIBA-treated plants (Figure 3.11A). Moreover, 35S:HA-ROXY19 plants were - like tga256 mutant plants - more sensitive to TIBA (Figure 3.11B). Consistently, the TGACGT motif was 2.5-fold enriched in the promoters of the downregulated genes. Five ATAF-type NAC transcription factors (ATAF1/ANAC002, ANAC014, NAC032, ANAC041 and ANAC102) were amongst the target genes of ROXY19. Since these are induced by xenobiotic stress, they might act as secondary transcription factors (Ratnakaran, 2014), which would explain the enrichment of the NAC binding sites in the target promoters. The strong down-regulation of the detoxification pathway might thus be the consequence of ROXY19 repressing TGA-factors at the TGACGT motifs of the promoters of 140 of the 337 (Figure 3.10B) genes and the consequence of reduced transcript levels of TGACGT-free promoters being regulated by ATAF-type transcription factors. It remains to be shown, whether the third class II TGA-regulated process, namely SAR, is also repressible by ROXY19. No hints can be derived from our microarray analysis since these genes might be expressed at too low levels, similar to ORA59, which is a target of ectopically expressed ROXY19 in ET-induced plants, but did not show reduced levels under the growth conditions used for our microarray analysis.

\subsection{The repressive effect of ROXY19 is not relieved in roxy19 mutants}

As outlined above, roxy18 plants are more resistant to the necrotrophic pathogen $B$. cinerea (La Camera et al., 2011), whereas no phenotype has yet been observed for roxy19 plants. Since ectopically expressed ROXY19 represses the JA-inducible CYP81D11 promoter and since ROXY19 is the only ROXY that is induced by JA, we tested whether JA-induced CYP81D11 expression would be hyper-induced in the roxy19 mutant. However, this was not the case (Figure 3.8C). Since ROXY19 is induced by xenobiotics and since it represses the xenobiotic response, one might have expected that mutation of roxy19 confers plants more resistant to high doses of xenobiotic treatment. In our growth assays both roxy19DS roxy18 double mutant and Nossen plants showed similar resistance to TIBA treatment (Figure 3.8G). Therefore, it has to be considered that other ROXYs containing an ALWL motif are highly expressed in leaves (e.g. ROXY4) and that JA- or TIBA-induced ROXY19 levels do not add considerably to the pool of ROXYs present in the leaf. Therefore, it remains to be explored whether higher-order ROXY mutants are necessary to validate their function in plants.

\subsection{Role of ROXY19 and TGAs for the crosstalk of SA- and ET/JA-signaling pathway}

The first report on the putative function of ROXY19 described that ROXY19 is induced by SA and that it represses JA-induced PDF1.2 expression (Ndamukong et al., 2007). Moreover, repression depends on class II TGA factors. Since SA suppresses JA-induced PDF1.2 expression and since this repression requires class II TGA factors, it has been proposed that SA induces ROXY19 expression, which subsequently inactivates class II TGA factors through reducing their Cys residue, leading to loss of ET/JA-induced maker genes expression, i.e. PDF1.2 (Caarls et al., 2015; Herrera-Vásquez et al., 2015). 
In agreement with this hypothesis, we found that the active site of ROXY19 was required for repressing ACC/JA-induced PDF1.2 (Supplemental Figure S4). However, previous experiments with the roxy19 and roxy18 roxy19 double mutant and even with the roxy18 roxy19 roxy20 triple mutant has shown that the negative effect of SA on JA- or ET-induced PDF1.2 expression is still functional (Zander, 2011). This might either show that ROXY19 does not play a role in the SA-ET/JA antagonism, or that another TGA-dependent mechanism is operational.

In this thesis, several experiments were performed to further investigate the role of TGA factors in the SA-ET/JA antagonism. Our complementation results showed that ET/JA-induced and SArepressed PDF1.2 expression was not altered at all in Arabidopsis transgenic plants expressing mutant TGA5 or TGA2 in which the cysteine residues had been changed against serine residues (Figure 3.19). This suggests that redox modifications of class II TGAs at potentially critical cysteine residues are not involved in regulating PDF1.2 expression.

Moreover, we found that the impaired PDF1.2 expression in the tga256 triple mutant was restored in tga14256 pentuple mutant (Figure 3.21). Since PDF1.2 expression is like wild-type in the tga14 mutant, it has to be concluded that class II TGA factors are bound to critical TGACG binding sites (e.g. in ORA59 or PDF1.2 promoter) to prevent access to the negative class I TGA factors.

The restored PDF1.2 expression in ACC/JA-treated tga12456 seedlings grown on MS-plates was not repressed by SA (Figure 3.21A). This is in accordance to previous results that SA-mediated repression is class II TGA-dependent (Leon-Reyes et al., 2010; Zander et al., 2010). However, in soil grown tga12456 plants, ACC-induced PDF1.2 was still significantly repressed by SA (Figure 3.21C). This kind of inconsistency needs further characterization.

\section{Outlook}

One of the most urgent questions is the role of ROXY19 in Arabidopsis. As discovered in this thesis, ROXY19 is induced upon xenobiotic stress and it is able to repress genes that are induced under these conditions. However, the roxy19 mutant did not show altered responses under these conditions. ROXY19 is also induced under numerous conditions related to biotic stress, like PAMP (Pathogen-associated molecular pattern) treatment, infections with Pseudomonas, in leaves establishing systemic acquired resistance and SA and JA treatment. At the moment, the roxy19 allele is in the Nossen background which contains genomic regions from Landsberg. Therefore, the results of pathogen infection assays might be influenced by the Landsberg introgression. Due to genome editing technologies, it is feasible to construct a roxy19 mutant that can be investigated with regard to the sensitivity of pathogens. These future studies should also include a roxy18 roxy19 double mutant. The second urgent question concerns the relevance of the active site which is important for repression as shown in this thesis. It remains to be investigated, whether glutathione binding, $\mathrm{Fe} / \mathrm{S}$ binding and/or oxidoreductase activity is required for the repressive effect. Since repression is still functional if a CPYC motif is present, it remains to be investigated why the CCMC motif is so conserved. 


\section{Bibliography}

Baerson, S.R., Sánchez-Moreiras, A., Pedrol-Bonjoch, N., Schulz, M., Kagan, I.A., Agarwal, A.K., Reigosa, M.J., and Duke, S.O. (2005). Detoxification and Transcriptome Response in Arabidopsis Seedlings Exposed to the Allelochemical Benzoxazolin-2(3H)-one. J. Biol. Chem. 280, 21867-21881.

Bandyopadhyay, S., Gama, F., Molina-Navarro, M.M., Gualberto, J.M., Claxton, R., Naik, S.G., Huynh, B.H., Herrero, E., Jacquot, J.P., Johnson, M.K., et al. (2008). Chloroplast monothiol glutaredoxins as scaffold proteins for the assembly and delivery of [2Fe-2S] clusters. EMBO J. 27, 1122-1133.

Behringer, C., Bartsch, K., and Schaller, A. (2011). Safeners recruit multiple signalling pathways for the orchestrated induction of the cellular xenobiotic detoxification machinery in Arabidopsis. Plant Cell Environ. 34, 1970-1985.

Bender, K.W., Wang, X., Cheng, G.B., Kim, H.S., Zielinski, R.E., and Huber, S.C. (2015). Glutaredoxin AtGRXC2 catalyses inhibitory glutathionylation of Arabidopsis BRI1-associated receptor-like kinase 1 (BAK1) in vitro. Biochem. J. 467, 399-413.

Berendzen, K.W., Weiste, C., Wanke, D., Kilian, J., Harter, K., and Dröge-Laser, W. (2012). Bioinformatic cis-element analyses performed in Arabidopsis and rice disclose bZIP- and MYB-related binding sites as potential AuxRE-coupling elements in auxin-mediated transcription. BMC Plant Biol. 12,125 .

Brandes, H.K., Larimer, F.W., Geck, M.K., Stringer, C.D., Schürmann, P., and Hartman, F.C. (1993). Direct identification of the primary nucleophile of thioredoxin f. J. Biol. Chem. 268, 18411-18414.

Caarls, L., Pieterse, C.M.J., and Van Wees, S.C.M. (2015). How salicylic acid takes transcriptional control over jasmonic acid signaling. Front. Plant Sci. 6, 170.

Chaubal, R., Anderson, J.R., Trimnell, M.R., Fox, T.W., Albertsen, M.C., and Bedinger, P. (2003). The transformation of anthers in the msca1 mutant of maize. Planta $216,778-788$.

Cheng, N.-H., Liu, J.-Z., Brock, A., Nelson, R.S., and Hirschi, K.D. (2006). AtGRXcp, an Arabidopsis chloroplastic glutaredoxin, is critical for protection against protein oxidative damage. J. Biol. Chem. $281,26280-26288$.

Cheng, N.-H., Liu, J.-Z., Liu, X., Wu, Q., Thompson, S.M., Lin, J., Chang, J., Whitham, S.A., Park, S., Cohen, J.D., et al. (2011). Arabidopsis monothiol glutaredoxin, AtGRXS17, is critical for temperaturedependent postembryonic growth and development via modulating auxin response. J. Biol. Chem. 286, 20398-20406.

Chini, A., Fonseca, S., Fernández, G., Adie, B., Chico, J.M., Lorenzo, O., García-Casado, G., LópezVidriero, I., Lozano, F.M., Ponce, M.R., et al. (2007). The JAZ family of repressors is the missing link in jasmonate signalling. Nature 448, 666-671.

Chomczynski, P. (1993). A reagent for the single-step simultaneous isolation of RNA, DNA and proteins from cell and tissue samples. BioTechniques 15, 532-534, 536-537.

Chuang, C.F., Running, M.P., Williams, R.W., and Meyerowitz, E.M. (1999). The PERIANTHIA gene encodes a bZIP protein involved in the determination of floral organ number in Arabidopsis thaliana. Genes Dev. 13, 334-344.

Clough, S.J., and Bent, A.F. (1998). Floral dip: a simplified method for Agrobacterium-mediated transformation of Arabidopsis thaliana. Plant J. Cell Mol. Biol. 16, 735-743. 
Couturier, J., Didierjean, C., Jacquot, J.-P., and Rouhier, N. (2010). Engineered mutated glutaredoxins mimicking peculiar plant class III glutaredoxins bind iron-sulfur centers and possess reductase activity. Biochem. Biophys. Res. Commun. 403, 435-441.

Couturier, J., Ströher, E., Albetel, A.-N., Roret, T., Muthuramalingam, M., Tarrago, L., Seidel, T., Tsan, P., Jacquot, J.-P., Johnson, M.K., et al. (2011). Arabidopsis Chloroplastic Glutaredoxin C5 as a Model to Explore Molecular Determinants for Iron-Sulfur Cluster Binding into Glutaredoxins. J. Biol. Chem. 286, 27515-27527.

Curtis, M.D., and Grossniklaus, U. (2003). A Gateway Cloning Vector Set for High-Throughput Functional Analysis of Genes in Planta. Plant Physiol. 133, 462-469.

Du, Z., Zhou, X., Ling, Y., Zhang, Z., and Su, Z. (2010). agriGO: a GO analysis toolkit for the agricultural community. Nucleic Acids Res. 38, W64-W70.

Fan, W., and Dong, X. (2002). In Vivo Interaction between NPR1 and Transcription Factor TGA2 Leads to Salicylic Acid-Mediated Gene Activation in Arabidopsis. Plant Cell 14, 1377-1389.

Feng, Y., Zhong, N., Rouhier, N., Hase, T., Kusunoki, M., Jacquot, J.-P., Jin, C., and Xia, B. (2006). Structural Insight into Poplar Glutaredoxin C1 with a Bridging Iron-Sulfur Cluster at the Active Site $†, \ddagger$. Biochemistry (Mosc.) 45, 7998-8008.

Fernandes, A.P., and Holmgren, A. (2004). Glutaredoxins: Glutathione-Dependent Redox Enzymes with Functions Far Beyond a Simple Thioredoxin Backup System. Antioxid. Redox Signal. 6, 63-74.

Fode, B., Siemsen, T., Thurow, C., Weigel, R., and Gatz, C. (2008). The Arabidopsis GRAS Protein SCL14 Interacts with Class II TGA Transcription Factors and Is Essential for the Activation of StressInducible Promoters. Plant Cell 20, 3122-3135.

Foley, R.C., and Singh, K.B. (2004). TGA5 acts as a positive and TGA4 acts as a negative regulator of ocs element activity in Arabidopsis roots in response to defence signals. FEBS Lett. 563, 141-145.

Fu, Z.Q., Yan, S., Saleh, A., Wang, W., Ruble, J., Oka, N., Mohan, R., Spoel, S.H., Tada, Y., Zheng, N., et al. (2012). NPR3 and NPR4 are receptors for the immune signal salicylic acid in plants. Nature 486, 228-232.

Garretón, V., Carpinelli, J., Jordana, X., and Holuigue, L. (2002). The as-1 Promoter Element Is an Oxidative Stress-Responsive Element and Salicylic Acid Activates It via Oxidative Species. Plant Physiol. $130,1516-1526$.

Hanson, G.T., Aggeler, R., Oglesbee, D., Cannon, M., Capaldi, R.A., Tsien, R.Y., and Remington, S.J. (2004). Investigating Mitochondrial Redox Potential with Redox-sensitive Green Fluorescent Protein Indicators. J. Biol. Chem. 279, 13044-13053.

Herrera-Vásquez, A., Carvallo, L., Blanco, F., Tobar, M., Villarroel-Candia, E., Vicente-Carbajosa, J., Salinas, P., and Holuigue, L. (2014). Transcriptional Control of Glutaredoxin GRXC9 Expression by a Salicylic Acid-Dependent and NPR1-Independent Pathway in Arabidopsis. Plant Mol. Biol. Report. 33, 624-637.

Herrera-Vásquez, A., Salinas, P., and Holuigue, L. (2015). Salicylic acid and reactive oxygen species interplay in the transcriptional control of defense genes expression. Front. Plant Sci. 6, 171. 
Ito, T., Motohashi, R., Kuromori, T., Mizukado, S., Sakurai, T., Kanahara, H., Seki, M., and Shinozaki, K. (2002). A New Resource of Locally Transposed Dissociation Elements for Screening Gene-Knockout Lines in Silico on the Arabidopsis Genome. Plant Physiol. 129, 1695-1699.

James, P., Halladay, J., and Craig, E.A. (1996). Genomic libraries and a host strain designed for highly efficient two-hybrid selection in yeast. Genetics 144, 1425-1436.

Kagale, S., and Rozwadowski, K. (2011). EAR motif-mediated transcriptional repression in plants. Epigenetics 6, 141-146.

Karimi, M., Inzé, D., and Depicker, A. (2002). GATEWAY ${ }^{\top M}$ vectors for Agrobacterium-mediated plant transformation. Trends Plant Sci. 7, 193-195.

Knuesting, J., Riondet, C., Maria, C., Kruse, I., Bécuwe, N., König, N., Berndt, C., Tourrette, S., Guilleminot-Montoya, J., Herrero, E., et al. (2015). Arabidopsis glutaredoxin S17 and its partner, the nuclear factor $Y$ subunit $\mathrm{C} 11 /$ negative cofactor $2 \alpha$, contribute to maintenance of the shoot apical meristem under long-day photoperiod. Plant Physiol. 167, 1643-1658.

Koncz, C., and Schell, J. (1986). The promoter of TL-DNA gene 5 controls the tissue-specific expression of chimaeric genes carried by a novel type of Agrobacterium binary vector. Mol. Gen. Genet. MGG 204, 383-396.

Koornneef, A., Rindermann, K., Gatz, C., and Pieterse, C.M. (2008). Histone modifications do not play a major role in salicylate-mediated suppression of jasmonate-induced PDF1.2 gene expression. Commun. Integr. Biol. 1, 143-145.

Köster, J., Thurow, C., Kruse, K., Meier, A., Iven, T., Feussner, I., and Gatz, C. (2012). Xenobiotic- and jasmonic acid-inducible signal transduction pathways have become interdependent at the Arabidopsis CYP81D11 promoter. Plant Physiol. 159, 391-402.

Krogan, N.T., Hogan, K., and Long, J.A. (2012). APETALA2 negatively regulates multiple floral organ identity genes in Arabidopsis by recruiting the co-repressor TOPLESS and the histone deacetylase HDA19. Dev. Camb. Engl. 139, 4180-4190.

Kuo, M.-H., and Allis, C.D. (1998). Roles of histone acetyltransferases and deacetylases in gene regulation. BioEssays 20, 615-626.

La Camera, S., L'haridon, F., Astier, J., Zander, M., Abou-Mansour, E., Page, G., Thurow, C., Wendehenne, D., Gatz, C., Métraux, J.-P., et al. (2011). The glutaredoxin ATGRXS13 is required to facilitate Botrytis cinerea infection of Arabidopsis thaliana plants. Plant J. Cell Mol. Biol. 68, 507-519.

Laporte, D., Olate, E., Salinas, P., Salazar, M., Jordana, X., and Holuigue, L. (2012). Glutaredoxin GRXS13 plays a key role in protection against photooxidative stress in Arabidopsis. J. Exp. Bot. 63, 503-515.

Leon-Reyes, A., Spoel, S.H., Lange, E.S.D., Abe, H., Kobayashi, M., Tsuda, S., Millenaar, F.F., Welschen, R.A.M., Ritsema, T., and Pieterse, C.M.J. (2009). Ethylene Modulates the Role of NONEXPRESSOR OF PATHOGENESIS-RELATED GENES1 in Cross Talk between Salicylate and Jasmonate Signaling. Plant Physiol. 149, 1797-1809.

Leon-Reyes, A., Du, Y., Koornneef, A., Proietti, S., Körbes, A.P., Memelink, J., Pieterse, C.M.J., and Ritsema, T. (2010). Ethylene Signaling Renders the Jasmonate Response of Arabidopsis Insensitive to Future Suppression by Salicylic Acid. Mol. Plant. Microbe Interact. 23, 187-197. 
Li, S., Lauri, A., Ziemann, M., Busch, A., Bhave, M., and Zachgo, S. (2009). Nuclear activity of ROXY1, a glutaredoxin interacting with TGA factors, is required for petal development in Arabidopsis thaliana. Plant Cell 21, 429-441.

Lillig, C.H., Berndt, C., and Holmgren, A. (2008). Glutaredoxin systems. Biochim. Biophys. Acta 1780, 1304-1317.

Long, J.A., Ohno, C., Smith, Z.R., and Meyerowitz, E.M. (2006). TOPLESS regulates apical embryonic fate in Arabidopsis. Science 312, 1520-1523.

Lu, J., and Holmgren, A. (2014). The thioredoxin superfamily in oxidative protein folding. Antioxid. Redox Signal. 21, 457-470.

Marty, L., Siala, W., Schwarzländer, M., Fricker, M.D., Wirtz, M., Sweetlove, L.J., Meyer, Y., Meyer, A.J., Reichheld, J.-P., and Hell, R. (2009). The NADPH-dependent thioredoxin system constitutes a functional backup for cytosolic glutathione reductase in Arabidopsis. Proc. Natl. Acad. Sci. 106, 91099114.

Meyer, A.J., Brach, T., Marty, L., Kreye, S., Rouhier, N., Jacquot, J.-P., and Hell, R. (2007). Redoxsensitive GFP in Arabidopsis thaliana is a quantitative biosensor for the redox potential of the cellular glutathione redox buffer. Plant J. 52, 973-986.

Moseler, A., Aller, I., Wagner, S., Nietzel, T., Przybyla-Toscano, J., Mühlenhoff, U., Lill, R., Berndt, C., Rouhier, N., Schwarzländer, M., et al. (2015). The mitochondrial monothiol glutaredoxin S15 is essential for iron-sulfur protein maturation in Arabidopsis thaliana. Proc. Natl. Acad. Sci. 201510835.

Mou, Z., Fan, W., and Dong, X. (2003). Inducers of plant systemic acquired resistance regulate NPR1 function through redox changes. Cell 113, 935-944.

Mueller, S., Hilbert, B., Dueckershoff, K., Roitsch, T., Krischke, M., Mueller, M.J., and Berger, S. (2008). General Detoxification and Stress Responses Are Mediated by Oxidized Lipids through TGA Transcription Factors in Arabidopsis. Plant Cell 20, 768-785.

Murmu, J., Bush, M.J., DeLong, C., Li, S., Xu, M., Khan, M., Malcolmson, C., Fobert, P.R., Zachgo, S., and Hepworth, S.R. (2010). Arabidopsis basic leucine-zipper transcription factors TGA9 and TGA10 interact with floral glutaredoxins ROXY1 and ROXY2 and are redundantly required for anther development. Plant Physiol. 154, 1492-1504.

Ndamukong, I.C. (2006). Characterization of an Arabidopsis glutaredoxin that interacts with core components of the salicylic acid signal transduction pathway. Its role in regulating the jasmonic acid pathway. PhD thesis.

Ndamukong, I., Abdallat, A.A., Thurow, C., Fode, B., Zander, M., Weigel, R., and Gatz, C. (2007). SAinducible Arabidopsis glutaredoxin interacts with TGA factors and suppresses JA-responsive PDF1.2 transcription. Plant J. Cell Mol. Biol. 50, 128-139.

Parisy, V., Poinssot, B., Owsianowski, L., Buchala, A., Glazebrook, J., and Mauch, F. (2007). Identification of PAD2 as a $\gamma$-glutamylcysteine synthetase highlights the importance of glutathione in disease resistance of Arabidopsis. Plant J. 49, 159-172.

Pautler, M., Eveland, A.L., LaRue, T., Yang, F., Weeks, R., Lunde, C., Je, B.I., Meeley, R., Komatsu, M., Vollbrecht, E., et al. (2015). FASCIATED EAR4 encodes a bZIP transcription factor that regulates shoot meristem size in maize. Plant Cell 27, 104-120. 
Pauwels, L., and Goossens, A. (2011). The JAZ Proteins: A Crucial Interface in the Jasmonate Signaling Cascade. Plant Cell 23, 3089-3100.

Pauwels, L., Barbero, G.F., Geerinck, J., Tilleman, S., Grunewald, W., Pérez, A.C., Chico, J.M., Bossche, R.V., Sewell, J., Gil, E., et al. (2010). NINJA connects the co-repressor TOPLESS to jasmonate signalling. Nature 464, 788-791.

Pieterse, C.M.J., Leon-Reyes, A., Van der Ent, S., and Van Wees, S.C.M. (2009). Networking by smallmolecule hormones in plant immunity. Nat. Chem. Biol. 5, 308-316.

Pontier, D., Privat, I., Trifa, Y., Zhou, J.-M., Klessig, D.F., and Lam, E. (2002). Differential regulation of TGA transcription factors by post-transcriptional control. Plant J. Cell Mol. Biol. 32, 641-653.

Poor, C.B., Wegner, S.V., Li, H., Dlouhy, A.C., Schuermann, J.P., Sanishvili, R., Hinshaw, J.R., RiggsGelasco, P.J., Outten, C.E., and He, C. (2014). Molecular mechanism and structure of the Saccharomyces cerevisiae iron regulator Aft2. Proc. Natl. Acad. Sci. U. S. A. 111, 4043-4048.

Qiao, H., Shen, Z., Huang, S.C., Schmitz, R.J., Urich, M.A., Briggs, S.P., and Ecker, J.R. (2012). Processing and Subcellular Trafficking of ER-Tethered EIN2 Control Response to Ethylene Gas. Science 338, 390-393.

Ramel, F., Sulmon, C., Serra, A.-A., Gouesbet, G., and Couée, I. (2012). Xenobiotic sensing and signalling in higher plants. J. Exp. Bot. ers102.

Ratnakaran, N. (2014). Identification of the role of Arabidopsis ATAF-type NAC transcription factors in plant stress and development. PhD thesis.

Riondet, C., Desouris, J.P., Montoya, J.G., Chartier, Y., Meyer, Y., and Reichheld, J.-P. (2012). A dicotyledon-specific glutaredoxin GRXC1 family with dimer-dependent redox regulation is functionally redundant with GRXC2. Plant Cell Environ. 35, 360-373.

Rouhier, N., Gelhaye, E., Sautiere, P.-E., Brun, A., Laurent, P., Tagu, D., Gerard, J., Faÿ, E. de, Meyer, Y., and Jacquot, J.-P. (2001). Isolation and Characterization of a New Peroxiredoxin from Poplar Sieve Tubes That Uses Either Glutaredoxin or Thioredoxin as a Proton Donor. Plant Physiol. 127, 12991309.

Rouhier, N., Gelhaye, E., and Jacquot, J.P. (2002). Glutaredoxin-dependent Peroxiredoxin from Poplar PROTEIN-PROTEIN INTERACTION AND CATALYTIC MECHANISM. J. Biol. Chem. 277, 13609-13614.

Rouhier, N., Gelhaye, E., and Jacquot, J.-P. (2004). Plant glutaredoxins: still mysterious reducing systems. Cell. Mol. Life Sci. CMLS 61, 1266-1277.

Rouhier, N., Villarejo, A., Srivastava, M., Gelhaye, E., Keech, O., Droux, M., Finkemeier, I., Samuelsson, G., Dietz, K.J., Jacquot, J.-P., et al. (2005). Identification of Plant Glutaredoxin Targets. Antioxid. Redox Signal. 7, 919-929.

Rouhier, N., Unno, H., Bandyopadhyay, S., Masip, L., Kim, S.-K., Hirasawa, M., Gualberto, J.M., Lattard, V., Kusunoki, M., Knaff, D.B., et al. (2007). Functional, structural, and spectroscopic characterization of a glutathione-ligated [2Fe-2S] cluster in poplar glutaredoxin C1. Proc. Natl. Acad. Sci. 104, 73797384.

Sambrook, J., and Russell, D.W. (2001). Molecular Cloning: A Laboratory Manual (CSHL Press).

Sandermann, H. (1992). Plant metabolism of xenobiotics. Trends Biochem. Sci. 17, 82-84. 
Schmittgen, T.D., and Livak, K.J. (2008). Analyzing real-time PCR data by the comparative CT method. Nat. Protoc. 3, 1101-1108.

Spoel, S.H., Johnson, J.S., and Dong, X. (2007). Regulation of tradeoffs between plant defenses against pathogens with different lifestyles. Proc. Natl. Acad. Sci. U. S. A. 104, 18842-18847.

Tamarit, J., Bellí, G., Cabiscol, E., Herrero, E., and Ros, J. (2003). Biochemical Characterization of Yeast Mitochondrial Grx5 Monothiol Glutaredoxin. J. Biol. Chem. 278, 25745-25751.

Thines, B., Katsir, L., Melotto, M., Niu, Y., Mandaokar, A., Liu, G., Nomura, K., He, S.Y., Howe, G.A., and Browse, J. (2007). JAZ repressor proteins are targets of the SCFCOI1 complex during jasmonate signalling. Nature 448, 661-665.

Van der Does, D., Leon-Reyes, A., Koornneef, A., Van Verk, M.C., Rodenburg, N., Pauwels, L., Goossens, A., Körbes, A.P., Memelink, J., Ritsema, T., et al. (2013). Salicylic acid suppresses jasmonic acid signaling downstream of SCFCOI1-JAZ by targeting GCC promoter motifs via transcription factor ORA59. Plant Cell 25, 744-761.

Wang, L., Kim, J., and Somers, D.E. (2013). Transcriptional corepressor TOPLESS complexes with pseudoresponse regulator proteins and histone deacetylases to regulate circadian transcription. Proc. Natl. Acad. Sci. U. S. A. 110, 761-766.

Wang, Z., Xing, S., Birkenbihl, R.P., and Zachgo, S. (2009). Conserved Functions of Arabidopsis and Rice CC-Type Glutaredoxins in Flower Development and Pathogen Response. Mol. Plant 2, 323-335.

Weiste, C., Iven, T., Fischer, U., Oñate-Sánchez, L., and Dröge-Laser, W. (2007). In planta ORFeome analysis by large-scale over-expression of GATEWAY ${ }^{\circledR}$-compatible cDNA clones: screening of ERF transcription factors involved in abiotic stress defense. Plant J. 52, 382-390.

Wu, Q., Lin, J., Liu, J.-Z., Wang, X., Lim, W., Oh, M., Park, J., Rajashekar, C.B., Whitham, S.A., Cheng, N.-H., et al. (2012). Ectopic expression of Arabidopsis glutaredoxin AtGRXS17 enhances thermotolerance in tomato. Plant Biotechnol. J. 10, 945-955.

Xing, S., and Zachgo, S. (2008). ROXY1 and ROXY2, two Arabidopsis glutaredoxin genes, are required for anther development. Plant J. Cell Mol. Biol. 53, 790-801.

Xing, S., Rosso, M.G., and Zachgo, S. (2005). ROXY1, a member of the plant glutaredoxin family, is required for petal development in Arabidopsis thaliana. Dev. Camb. Engl. 132, 1555-1565.

Yang, F., Bui, H.T., Pautler, M., Llaca, V., Johnston, R., Lee, B., Kolbe, A., Sakai, H., and Jackson, D. (2015). A maize glutaredoxin gene, abphyl2, regulates shoot meristem size and phyllotaxy. Plant Cell $27,121-131$.

Yoo, S.-D., Cho, Y.-H., and Sheen, J. (2007). Arabidopsis mesophyll protoplasts: a versatile cell system for transient gene expression analysis. Nat. Protoc. 2, 1565-1572.

Zander, M. (2011). Arabidopsis thaliana class II TGA transcription factors provide a molecular link between salicylic acid and ethylene defense signalling. PhD thesis.

Zander, M., La Camera, S., Lamotte, O., Métraux, J.-P., and Gatz, C. (2010). Arabidopsis thaliana classII TGA transcription factors are essential activators of jasmonic acid/ethylene-induced defense responses. Plant J. Cell Mol. Biol. 61, 200-210. 
Zander, M., Chen, S., Imkampe, J., Thurow, C., and Gatz, C. (2012). Repression of the Arabidopsis thaliana jasmonic acid/ethylene-induced defense pathway by TGA-interacting glutaredoxins depends on their C-terminal ALWL motif. Mol. Plant 5, 831-840.

Zander, M., Thurow, C., and Gatz, C. (2014). TGA Transcription Factors Activate the Salicylic AcidSuppressible Branch of the Ethylene-Induced Defense Program by Regulating ORA59 Expression. Plant Physiol. 165, 1671-1683.

Zhou, C., Zhang, L., Duan, J., Miki, B., and Wu, K. (2005). HISTONE DEACETYLASE19 is involved in jasmonic acid and ethylene signaling of pathogen response in Arabidopsis. Plant Cell 17, 1196-1204.

Zhu, Z., An, F., Feng, Y., Li, P., Xue, L., A, M., Jiang, Z., Kim, J.-M., To, T.K., Li, W., et al. (2011). Derepression of ethylene-stabilized transcription factors (EIN3/EIL1) mediates jasmonate and ethylene signaling synergy in Arabidopsis. Proc. Natl. Acad. Sci. 108, 12539-12544. 


\section{Abbreviations}

$\%$

${ }^{\circ} \mathrm{C}$

$\mu l$

$\mu \mathrm{M}$

5-azaC

A. thaliana

A. tumefaciens

$\mathrm{ABC}$

ACC

$A D$

AGI

as-1

ATAF

B. cinerea

BHA

BOA

bZIP

CAT

CDNA

ChIP

$\mathrm{cm}$

COI1

Col

COR78

$\mathrm{C}_{\mathrm{t}}$

CUC

CYP

DBD

DMSO

DMTU

DNA

DNase

dNTP

dpi

DS

E.coli

e.g.

EAR

EDTA

EIN3

ERF

$\beta$-est

ET

EtOH

FC

FEA4

Fe-S

fwd

GO

GR

GRAS

GRX

GSH

GST

GUS

GW
Percent

degree Celsius

microliter

micromolar

5-Azacytidine

Arabidopsis thaliana

Agrobacterium tumefaciens

ATP-binding cassette

1-aminocyclopropane-1-carboxylic acid

activation domain

Arabidopsis genome initiative

activation sequence-1

ARABIDOPSIS THALIANA ACTIVATION FACTOR

Botrytis cinerea

butylated hydroxyanisole

benzoxazolin-2(3H)-one

basic domain/leucine zipper

Catalase

complementary DNA

chromatin immunoprecipitation

centimeter

CORONATINE INSENSITIVE 1

Columbia

COLD REGULATED 78

cycle threshold

CUP-SHAPED COTYLEDON

Cytochrome P450

DNA-binding domain

dimethyl sulfoxide

dimethylthiourea

deoxyribonucleic acid

deoxyribonuclease

deoxynucleoside triphosphate

day(s) post infection

dissociation

Escherichia coli

for example (exempli gratia)

ERF-associated amphiphilic repression

ethylene di-amine tetra-acetic acid

ETHYLENE INSENSITIVE 3

APETALA2/ETHYLENE RESPONSIVE FACTOR

$\beta$-estradiol

ethylene

ethanol

fold change

FASCIATED EAR4

Iron-sulfur

forward

Gene Ontology

Glutathione reductase

GAI, RGA, SCR

Glutaredoxin

Glutathione

Glutathione S-Transferases

$\beta$-glucuronidase

gateway 


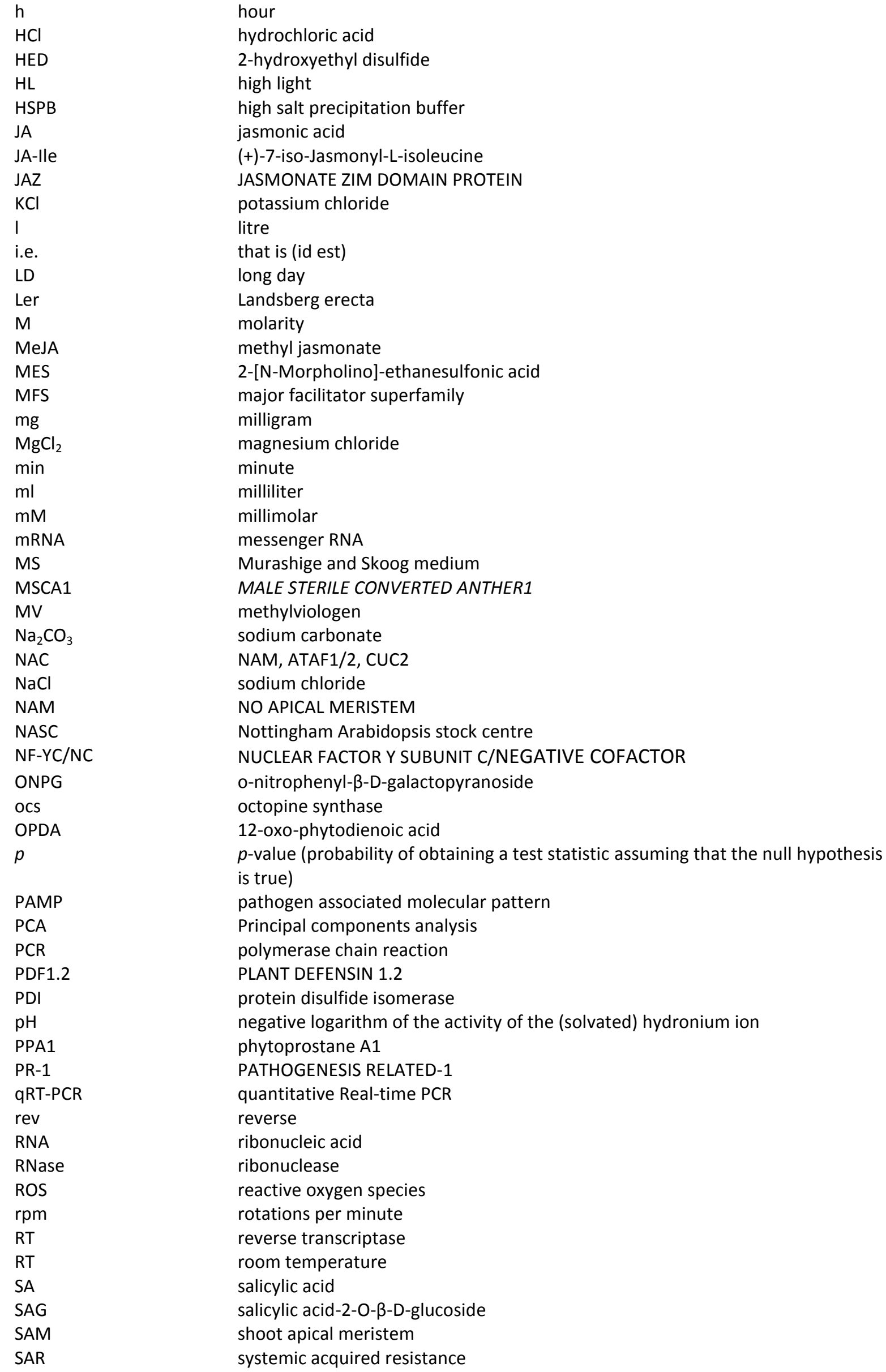


SCL14

SD

SDS

SE

$\mathrm{Sec}$

taq

TAIR

TF

TGA

TIBA

TPL/TPR

TRX

UBQ5

UGT

w/v

WT

YPAD
SCARECROW LIKE-14

short day

sodium dodecylsulfate

standard error of mean

second

Thermus aquaticus

the Arabidopsis information resource

transcription factor

TGACG motif binding protein

2,3,5-Triiodobenzoic acid

TOPLESS/TOPLESS-RELATED PROTEINS

thioredoxin

UBIQUITIN-5

uridine-diphospho-glucuronosyltransferases

weight per volume

wild-type

Yeast extract-peptone-adenine-dextrose 


\section{Supplemental data}
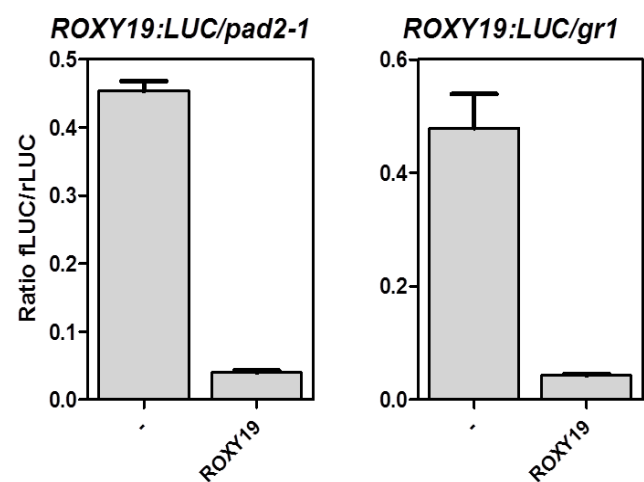

Figure S1 ROXY19 represses its own promoter in protoplast derived from mutant plants with altered GSH. Transient expression assays with ROXY19 effectors and the ROXY19 promoter in pad2-1 and gr1 mutant protoplasts. Expression of the ROXY19 promoter fused to the fLuc was analyzed in transiently transformed mesophyll protoplasts prepared from pad2-1 or gr1 mutant in the presence of effector plasmid encoding ROXY19 under the control of the UBQ10 promoter. An empty plasmid was used when effector plasmid was absent (-). Luc activities were determined $16 \mathrm{~h}$ after transfection. Values are means of four replicates $( \pm \mathrm{SE})$.

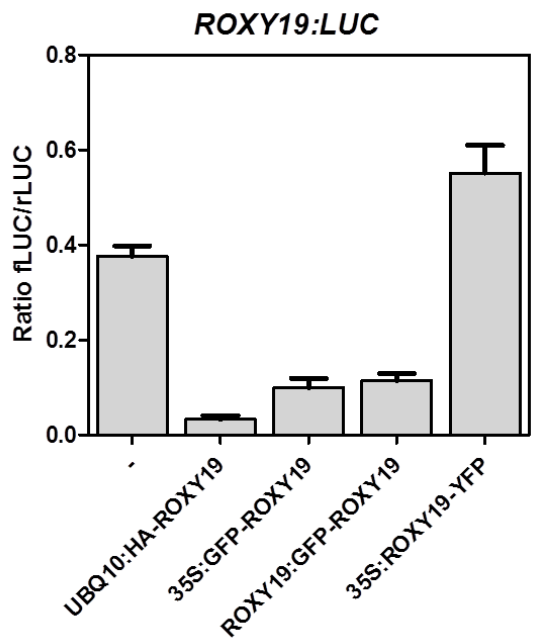

Figure S2 ROXY19 represses its own promoter requires the C-terminal ALWL motif. Transient expression assays with the ROXY19 promoter and ROXY19 effectors protoplasts. Expression of the ROXY19 promoter fused to the $f L u c$ was analyzed in transiently transformed mesophyll protoplasts in the presence of effector plasmids encoding HA-, GFP- or YFP-tagged ROXY19 controlled by UBQ10, 35S or ROXY19 promoter as indicated. An empty plasmid was used when effector plasmid was absent (-). Luc activities were determined $16 \mathrm{~h}$ after transfection. Values are means of four replicates $( \pm S E)$. 

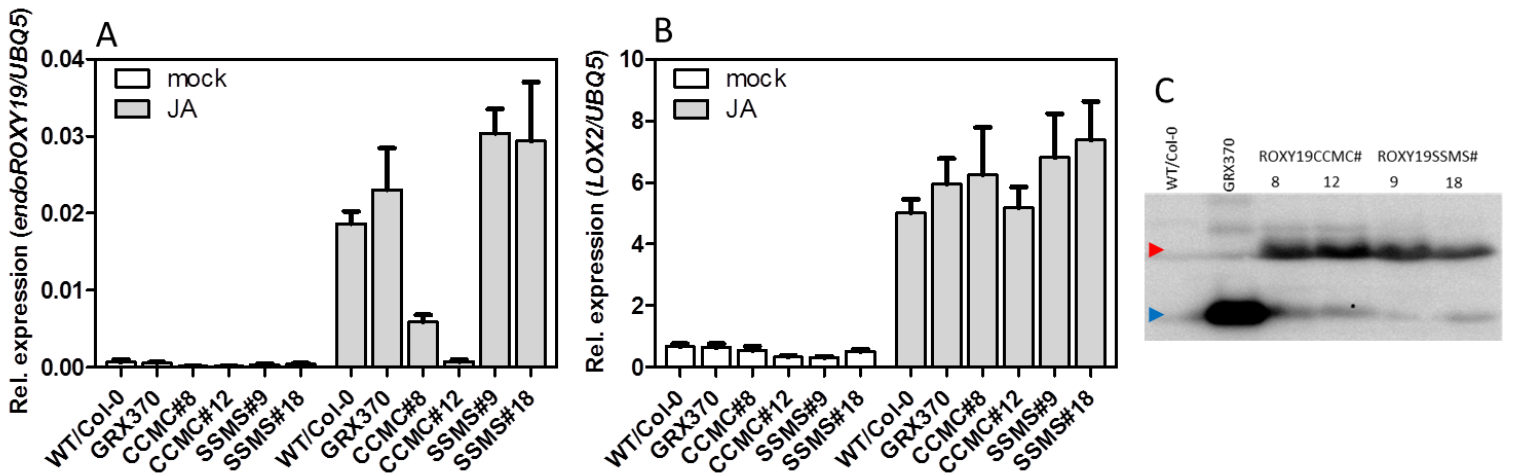

Figure S3 The active site is required for ROXY19 to repress JA-induced endogenous ROXY19 expression in transgenic plants. (A) and (B) qRT-PCR analysis of endogenous ROXY19 and LOX2 expression in Col-0 and different transgenic plants after JA-treatment. Arabidopsis were grown on soil for four weeks, and subsequently treated with volatile JA (MeJA) in glass aquaria. After $10 \mathrm{~h}$ of treatment, plant leaves were harvested for RNA or protein extraction, endogenous ROXY19 and LOX2 transcripts were analyzed by qRT-PCR. The mean values $( \pm S E$ ) from 4-5 independent replicates (one pot with one plant as one biological replicate) are shown. (B) Western blot analysis of transgenic lines using antibody against HA tag. Protein was extracted from mock plant samples used for RNA extraction in Figure S3 (A) and (B). Red and blue arrowheads denote specific ROXY19 and GRX370 bands, respectively.

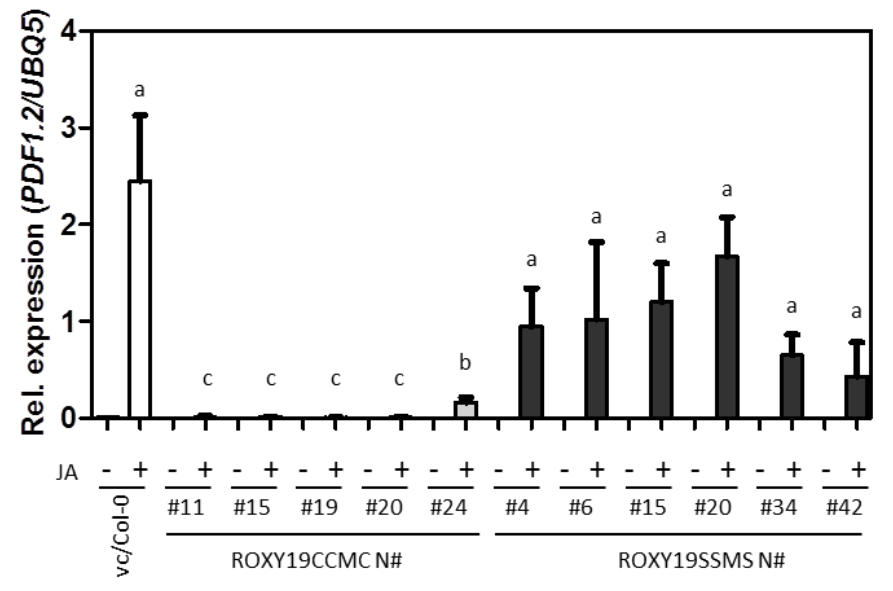

Figure S4 Expression of PDF1.2 in 35S:HA-ROXY19CCMC and 35S:HA-ROXY19SSMS plants after JA treatment. Samples were the same as used in Figure 3.6A. Homozygous Arabidopsis seeds from the T2 generation were germinated and grown on soil for four weeks, and subsequently treated with volatile JA (MeJA) in glass aquaria. After $10 \mathrm{~h}$ of treatment, plant leaves were harvested for RNA or protein extraction, PDF1.2 transcripts were analyzed by $q R T-P C R$. The mean values $( \pm S E)$ from four independent replicates (one pot with one plant as one biological replicate) of JA-treated samples are shown. One replicate of the corresponding mock sample is shown. Different letters indicate significant difference among genotypes after treatment (Student's $t$-test, $\mathrm{p}<0.05)$. White, gray and black bars indicate empty vector, WT and active site mutant ROXY19 transformed Col0 samples, respectively. 


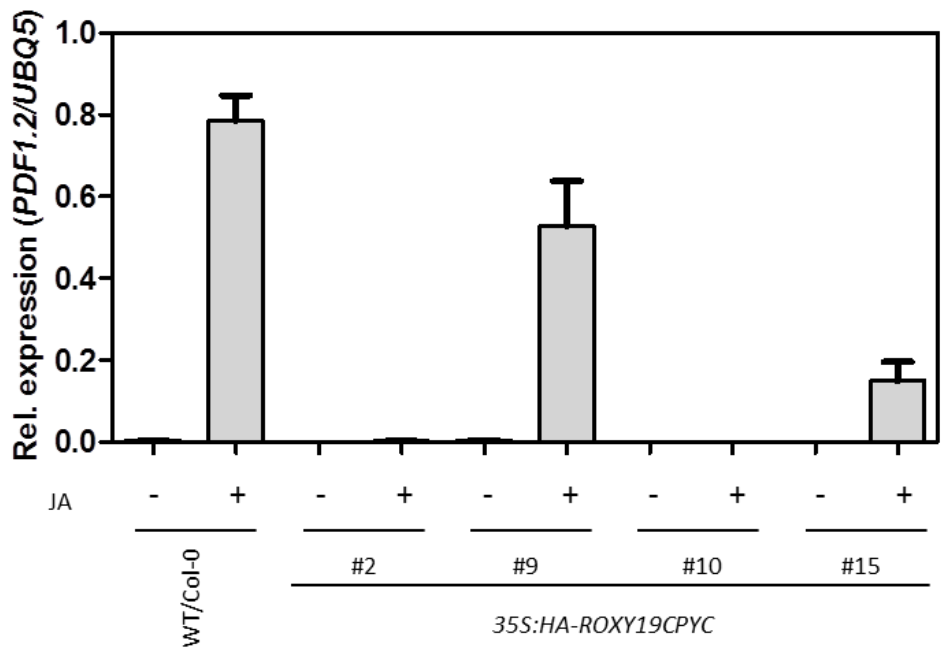

Figure S5 Expression PDF1.2 in 35S:HA-ROXY19CPYC lines after JA treatment. qRT-PCR analysis of PDF1.2 expression in Col-0 and 35S:HA-ROXY19CPYC transgenic plants after JA-treatment. RNA samples were the same used for analysis in Figure 3.7. Homozygous Arabidopsis seeds from the T2 generation were germinated and grown on soil pot for four weeks, and subsequently treated with volatile JA (MeJA) in glass aquaria. After $10 \mathrm{~h}$ of treatment, plant leaves were harvested for RNA or protein extraction, PDF1.2 transcripts are analyzed by qRT-PCR. The mean values $( \pm S E)$ from four independent replicates (one pot with one plant as one biological replicate) are shown. 
Table S1 List of genes down-regulated in 35S:HA-ROXY19CCMC\#8 plants (FC>1.5, $p<0.05)$.

\begin{tabular}{|c|c|c|c|c|c|c|c|c|}
\hline \multirow{2}{*}{ AGI code } & \multirow{2}{*}{ Description } & \multicolumn{5}{|c|}{ mean of linear expression values } & \multirow{2}{*}{$\begin{array}{l}\text { Col-0/CCMC\#8 } \\
\text { (fold repression) }\end{array}$} & \multirow{2}{*}{$p$-value } \\
\hline & & Col-0 & GRX370\#1 & SSMS\#9 & CCMC\#8 & CCMC\#12 & & \\
\hline AT1G03850 & Glutaredoxin family protein, ROXY18 & 161.7 & 193.5 & 128.1 & 14.2 & 15.4 & 11.37 & $2.99 \mathrm{E}-11$ \\
\hline AT3G28740 & CYP81D1 & 682.4 & 612.7 & 784.9 & 136.1 & 399.4 & 5.02 & $1.05 \mathrm{E}-11$ \\
\hline AT5G38910 & RmIC-like cupins superfamily protein & 118.2 & 401.6 & 148.4 & 24.6 & 16.0 & 4.81 & 2.95E-09 \\
\hline AT2G43510 & ATTI1, TI1 & 112.3 & 137.1 & 102.0 & 24.2 & 29.9 & 4.63 & $5.64 \mathrm{E}-08$ \\
\hline AT5G16980 & Zinc-binding dehydrogenase family protein & 49.8 & 48.2 & 37.4 & 10.8 & 32.0 & 4.62 & $9.21 \mathrm{E}-10$ \\
\hline AT1G55850 & ATCSLE1, CSLE1 & 434.8 & 423.6 & 397.4 & 110.7 & 250.2 & 3.93 & $7.18 \mathrm{E}-12$ \\
\hline AT4G11650 & ATOSM34, OSM34 & 648.0 & 1009.4 & 783.8 & 169.6 & 146.6 & 3.82 & $4.49 \mathrm{E}-11$ \\
\hline AT5G25130 & CYP71B12 & 133.5 & 128.8 & 109.2 & 36.3 & 75.5 & 3.68 & $7.42 \mathrm{E}-09$ \\
\hline AT4G12290 & Copper amine oxidase family protein & 294.7 & 391.9 & 320.0 & 81.4 & 195.4 & 3.62 & $3.75 \mathrm{E}-10$ \\
\hline AT1G05560 & UGT1, UGT75B1 & 243.4 & 186.1 & 243.6 & 69.0 & 131.4 & 3.53 & 7.33E-09 \\
\hline AT2G37770 & NAD(P)-linked oxidoreductase superfamily & 217.8 & 202.2 & 207.3 & 62.3 & 99.4 & 3.49 & $5.14 \mathrm{E}-06$ \\
\hline AT5G16970 & AT-AER, AER & 112.4 & 104.0 & 101.8 & 32.2 & 75.0 & 3.48 & 4.49E-09 \\
\hline AT3G11340 & UDP-Glycosyltransferase superfamily protein & 125.3 & 136.3 & 137.8 & 37.0 & 32.1 & 3.39 & 5.29E-08 \\
\hline AT1G02850 & BGLU11 & 314.3 & 260.6 & 329.3 & 96.7 & 153.5 & 3.25 & $8.45 \mathrm{E}-07$ \\
\hline AT4G23700 & ATCHX17, CHX17 & 81.5 & 140.7 & 92.1 & 25.4 & 21.0 & 3.21 & 5.07E-09 \\
\hline AT1G07900 & LBD1 & 36.5 & 62.6 & 30.0 & 11.5 & 22.2 & 3.16 & 0.000987 \\
\hline AT1G78670 & ATGGH3, GGH3 & 548.1 & 609.3 & 507.6 & 173.7 & 329.0 & 3.16 & $1.48 \mathrm{E}-13$ \\
\hline AT5G39050 & HXXXD-type acyl-transferase family protein & 336.9 & 288.5 & 338.2 & 107.6 & 184.3 & 3.13 & 5.09E-09 \\
\hline AT5G23760 & Copper transport protein family & 55.3 & 52.7 & 43.3 & 17.7 & 32.0 & 3.12 & 7.03E-08 \\
\hline AT1G67550 & URE & 100.4 & 102.3 & 84.3 & 32.2 & 62.0 & 3.12 & $5.23 \mathrm{E}-10$ \\
\hline AT1G26240 & Proline-rich extensin-like family protein & 55.4 & 49.8 & 67.9 & 17.8 & 10.5 & 3.11 & 9.77E-05 \\
\hline AT4G19880 & Glutathione S-transferase family protein & 35.6 & 22.4 & 20.2 & 11.4 & 14.2 & 3.11 & 0.00232 \\
\hline AT5G62760 & $\begin{array}{l}\text { P-loop containing nucleoside triphosphate } \\
\text { hydrolases superfamily protein }\end{array}$ & 16.4 & 8.1 & 8.7 & 5.5 & 5.4 & 3.02 & 0.006887 \\
\hline AT5G13750 & ZIFL1 & 269.0 & 275.0 & 281.7 & 89.3 & 136.8 & 3.01 & $2.17 \mathrm{E}-09$ \\
\hline AT3G21250 & MRP6, ABCC8 & 417.6 & 431.1 & 400.7 & 139.7 & 267.6 & 2.99 & $1.86 \mathrm{E}-08$ \\
\hline AT4G15530 & PPDK & 12.2 & 8.4 & 10.9 & 4.1 & 6.2 & 2.94 & 0.011416 \\
\hline AT4G12480 & pEARLI 1 & 161.1 & 189.3 & 94.2 & 54.8 & 94.3 & 2.94 & 0.005628 \\
\hline AT1G09160 & Protein phosphatase $2 \mathrm{C}$ family protein & 28.8 & 27.1 & 35.5 & 9.9 & 23.1 & 2.92 & 0.014611 \\
\hline AT2G36750 & UGT73C1 & 73.7 & 64.5 & 68.9 & 25.4 & 36.4 & 2.91 & 4.44E-07 \\
\hline AT1G64780 & ATAMT1 & 111.1 & 118.6 & 108.0 & 38.6 & 38.7 & 2.88 & $2.56 \mathrm{E}-08$ \\
\hline AT4G31970 & CYP82C2 & 94.1 & 119.4 & 126.1 & 32.8 & 24.5 & 2.87 & 0.000134 \\
\hline AT4G15550 & IAGLU & 226.8 & 224.9 & 220.8 & 80.3 & 140.4 & 2.83 & $2.89 \mathrm{E}-05$ \\
\hline AT2G26020 & PDF1.2b & 16.7 & 14.4 & 10.0 & 6.0 & 8.3 & 2.81 & 0.000544 \\
\hline AT5G24210 & alpha/beta-Hydrolases superfamily protein & 40.2 & 52.4 & 44.7 & 14.6 & 32.1 & 2.75 & 2.17E-05 \\
\hline AT1G14520 & MIOX1 & 48.6 & 45.9 & 46.1 & 18.0 & 25.2 & 2.69 & 4.29E-06 \\
\hline AT2G36800 & DOGT1, UGT73C5 & 195.7 & 192.8 & 224.8 & 73.1 & 141.4 & 2.68 & 7.63E-06 \\
\hline AT4G15530 & PPDK & 25.0 & 57.7 & 18.5 & 9.3 & 18.3 & 2.67 & 0.007228 \\
\hline AT4G23060 & IQD22 & 131.6 & 109.5 & 123.9 & 49.3 & 76.8 & 2.67 & $2.43 \mathrm{E}-09$ \\
\hline AT1G05570 & CALS1, GSL06, ATGSL6, ATGSL06, GSL6 & 150.7 & 220.9 & 144.6 & 56.5 & 143.0 & 2.67 & 0.001673 \\
\hline AT1G68540 & NAD(P)-binding Rossmann-fold superfamily & 61.8 & 74.1 & 46.4 & 23.5 & 56.1 & 2.63 & 0.000265 \\
\hline AT1G66783 & MIR157A & 18.6 & 14.1 & 17.6 & 7.1 & 15.1 & 2.63 & $6.11 \mathrm{E}-05$ \\
\hline AT4G37520 & Peroxidase superfamily protein & 65.4 & 82.9 & 67.2 & 25.1 & 24.2 & 2.60 & $2.05 \mathrm{E}-11$ \\
\hline AT3G18100 & MYB4R1, AtMYB4R1 & 15.2 & 13.4 & 10.5 & 5.9 & 5.3 & 2.59 & 0.013636 \\
\hline AT3G61880 & CYP78A9 & 43.5 & 42.8 & 38.6 & 16.8 & 27.1 & 2.58 & 0.000725 \\
\hline AT2G47890 & $\begin{array}{l}\text { B-box type zinc finger protein with CCT } \\
\text { domain }\end{array}$ & 444.8 & 462.8 & 442.9 & 175.1 & 270.6 & 2.54 & $6.2 \mathrm{E}-10$ \\
\hline AT3G04000 & NAD(P)-binding Rossmann-fold superfamily & 88.9 & 69.2 & 107.5 & 35.1 & 50.8 & 2.53 & $3.28 \mathrm{E}-06$ \\
\hline AT4G19810 & $\begin{array}{l}\text { Glycosyl hydrolase family protein with } \\
\text { chitinase insertion domain }\end{array}$ & 164.7 & 263.7 & 156.4 & 65.7 & 80.0 & 2.51 & 4.35E-08 \\
\hline AT1G26770 & ATEXPA10 & 89.2 & 81.5 & 66.7 & 35.8 & 49.0 & 2.49 & $1.38 \mathrm{E}-05$ \\
\hline AT5G61820 & FUNCTIONS IN: molecular_function unknown & 786.5 & 894.0 & 748.2 & 320.2 & 493.2 & 2.46 & $2.79 \mathrm{E}-09$ \\
\hline AT1G69440 & AGO7, ZIP & 79.4 & 61.8 & 73.0 & 32.7 & 49.7 & 2.43 & $2.85 \mathrm{E}-06$ \\
\hline AT1G26770 & ATEXPA10 & 342.3 & 344.7 & 263.3 & 141.6 & 192.6 & 2.42 & $4.83 \mathrm{E}-05$ \\
\hline AT1G17170 & ATGSTU24, GST, GSTU24 & 101.3 & 104.8 & 121.8 & 42.4 & 75.5 & 2.39 & 0.000185 \\
\hline AT1G68570 & Major facilitator superfamily protein & 315.1 & 406.4 & 246.1 & 132.3 & 212.1 & 2.38 & 0.000432 \\
\hline AT4G23680 & $\begin{array}{l}\text { Polyketide cyclase/dehydrase and lipid } \\
\text { transport superfamily protein }\end{array}$ & 226.6 & 288.4 & 164.0 & 95.8 & 144.4 & 2.37 & $2.63 \mathrm{E}-06$ \\
\hline AT4G14040 & EDA38, SBP2 & 518.7 & 444.4 & 471.5 & 219.8 & 297.3 & 2.36 & $2.32 \mathrm{E}-10$ \\
\hline AT2G04270 & RNEE/G & 7.0 & 3.8 & 3.9 & 3.0 & 3.7 & 2.34 & 0.020363 \\
\hline AT5G51830 & pfkB-like carbohydrate kinase family protein & 87.7 & 93.2 & 96.7 & 37.6 & 57.0 & 2.33 & $7.54 \mathrm{E}-07$ \\
\hline AT4G25360 & TBL18 & 15.2 & 11.8 & 10.9 & 6.5 & 12.2 & 2.33 & 0.005127 \\
\hline AT3G43930 & BRCT domain-containing DNA repair protein & 9.1 & 5.9 & 7.8 & 3.9 & 6.1 & 2.33 & 0.001244 \\
\hline AT1G08270 & $\begin{array}{l}\text { CONTAINS InterPro DOMAIN/s: MIT } \\
\text { (InterPro:IPR007330) }\end{array}$ & 8.4 & 4.4 & 5.4 & 3.6 & 5.6 & 2.33 & 0.021634 \\
\hline AT1G11310 & MLO2, ATMLO2, PMR2 & 8.8 & 11.1 & 9.9 & 3.8 & 4.6 & 2.32 & 0.00735 \\
\hline AT3G44300 & NIT2, AtNIT2 & 41.1 & 48.4 & 37.7 & 17.8 & 18.9 & 2.32 & $3.69 \mathrm{E}-08$ \\
\hline AT1G55500 & ECT4 & 105.6 & 70.8 & 81.3 & 45.8 & 59.5 & 2.30 & 0.004618 \\
\hline AT3G59140 & ATMRP14, MRP14, ABCC10 & 123.2 & 123.1 & 121.2 & 53.7 & 97.8 & 2.29 & $1.74 \mathrm{E}-07$ \\
\hline AT1G01720 & ATAF1, ANAC002 & 281.1 & 285.5 & 275.6 & 123.5 & 173.7 & 2.28 & $4.45 \mathrm{E}-09$ \\
\hline AT1G34220 & $\begin{array}{l}\text { Regulator of Vps } 4 \text { activity in the MVB } \\
\text { pathway protein }\end{array}$ & 17.0 & 8.8 & 16.4 & 7.6 & 16.2 & 2.25 & 0.030162 \\
\hline AT1G15125 & $\begin{array}{l}\text { S-adenosyl-L-methionine-dependent } \\
\text { methyltransferases superfamily protein }\end{array}$ & 232.6 & 155.0 & 222.7 & 103.4 & 224.3 & 2.25 & 0.021521 \\
\hline AT5G52800 & DNA primases & 8.8 & 8.1 & 4.6 & 3.9 & 5.0 & 2.24 & 0.018829 \\
\hline
\end{tabular}




\begin{tabular}{|c|c|c|c|c|c|c|c|c|}
\hline AT1G77450 & anac032, NAC032 & 257.3 & 238.2 & 227.9 & 115.3 & 160.2 & 2.23 & $1.29 \mathrm{E}-07$ \\
\hline AT4G13180 & NAD(P)-binding Rossmann-fold superfamily & 207.7 & 225.6 & 213.8 & 93.9 & 110.4 & 2.21 & $2.95 \mathrm{E}-06$ \\
\hline AT3G45060 & ATNRT2.6, NRT2.6 & 14.1 & 11.7 & 13.7 & 6.4 & 8.6 & 2.21 & $6.36 \mathrm{E}-05$ \\
\hline AT4G01070 & GT72B1, UGT72B1 & 624.6 & 801.4 & 622.9 & 284.3 & 358.9 & 2.20 & $3.68 \mathrm{E}-07$ \\
\hline AT5G63790 & ANAC102, NAC102 & 517.8 & 547.5 & 561.3 & 235.7 & 372.1 & 2.20 & $4.84 \mathrm{E}-09$ \\
\hline AT1G12200 & Flavin-binding monooxygenase family & 94.5 & 86.6 & 71.7 & 43.0 & 70.4 & 2.19 & $3.42 \mathrm{E}-08$ \\
\hline AT4G10970 & unknown protein & 23.4 & 21.0 & 19.8 & 10.7 & 18.5 & 2.19 & 0.048218 \\
\hline AT3G56080 & $\begin{array}{l}\text { S-adenosyl-L-methionine-dependent } \\
\text { methyltransferases superfamily protein }\end{array}$ & 55.1 & 74.3 & 51.6 & 25.3 & 32.0 & 2.17 & $2.55 \mathrm{E}-05$ \\
\hline AT1G76690 & OPR2, ATOPR2 & 53.2 & 50.5 & 45.5 & 24.5 & 36.4 & 2.17 & $2.83 \mathrm{E}-06$ \\
\hline AT3G14660 & CYP72A13 & 46.0 & 49.2 & 45.9 & 21.3 & 35.7 & 2.17 & $2.39 \mathrm{E}-08$ \\
\hline AT1G55900 & TIM50, emb1860 & 8.1 & 4.3 & 5.1 & 3.8 & 7.0 & 2.15 & 0.033372 \\
\hline AT2G37760 & NAD(P)-linked oxidoreductase superfamily & 191.1 & 165.0 & 168.8 & 89.3 & 123.5 & 2.14 & $1.64 \mathrm{E}-07$ \\
\hline AT5G50200 & WR3 & 114.1 & 116.0 & 104.8 & 53.3 & 57.7 & 2.14 & $9.28 \mathrm{E}-08$ \\
\hline AT4G04450 & WRKY42, AtWRKY42 & 46.5 & 47.5 & 47.0 & 21.7 & 23.3 & 2.14 & $5.48 \mathrm{E}-05$ \\
\hline AT5G23360 & $\begin{array}{l}\text { GRAM domain-containing protein / ABA- } \\
\text { responsive protein-related }\end{array}$ & 13.9 & 12.8 & 15.2 & 6.5 & 13.1 & 2.14 & 0.000348 \\
\hline AT5G36130 & Cytochrome P450 superfamily protein & 51.7 & 63.6 & 50.9 & 24.3 & 19.6 & 2.13 & 0.000171 \\
\hline AT5G26000 & TGG1, BGLU38 & 4297.1 & 5030.4 & 3892.6 & 2019.1 & 3137.7 & 2.13 & $1.06 \mathrm{E}-09$ \\
\hline AT5G36140 & CYP716A2 & 46.7 & 61.8 & 49.2 & 22.0 & 10.6 & 2.12 & 0.000664 \\
\hline AT1G76680 & OPR1 & 31.0 & 30.5 & 33.6 & 14.7 & 23.0 & 2.12 & $2.48 \mathrm{E}-06$ \\
\hline AT4G15530 & PPDK & 128.1 & 135.5 & 138.2 & 60.7 & 82.9 & 2.11 & $1.2 \mathrm{E}-05$ \\
\hline AT4G02280 & SUS3, ATSUS3 & 58.6 & 67.6 & 46.4 & 27.8 & 31.5 & 2.11 & 0.000101 \\
\hline AT5G50200 & WR3, ATNRT3.1, NRT3.1 & 11.3 & 13.3 & 11.5 & 5.4 & 4.8 & 2.10 & 0.004031 \\
\hline AT1G17860 & Kunitz family trypsin and protease inhibitor & 377.2 & 403.0 & 373.4 & 179.9 & 162.4 & 2.10 & $4.02 \mathrm{E}-07$ \\
\hline AT1G07570 & APK1A, APK1 & 10.0 & 10.6 & 14.6 & 4.8 & 7.3 & 2.09 & 0.018857 \\
\hline AT2G40316 & FUNCTIONS IN: molecular_function unknown & 10.8 & 7.6 & 8.8 & 5.2 & 9.4 & 2.09 & 0.010454 \\
\hline AT1G21680 & DPP6 N-terminal domain-like protein & 847.4 & 807.3 & 887.0 & 406.4 & 608.1 & 2.08 & $8.83 \mathrm{E}-09$ \\
\hline AT3G07180 & GPI transamidase component PIG-S-related & 16.3 & 14.3 & 10.1 & 7.9 & 9.4 & 2.07 & 0.036169 \\
\hline AT5G67430 & $\begin{array}{l}\text { Acyl-CoA N-acyltransferases (NAT) } \\
\text { superfamily protein }\end{array}$ & 77.1 & 82.3 & 69.9 & 37.7 & 35.4 & 2.05 & $1.23 \mathrm{E}-05$ \\
\hline AT4G34135 & UGT73B2 & 59.8 & 46.4 & 65.0 & 29.4 & 42.9 & 2.04 & 0.000939 \\
\hline AT3G28007 & SWEET4, AtSWEET4 & 23.6 & 38.0 & 17.2 & 11.6 & 11.3 & 2.03 & 0.014064 \\
\hline AT3G50650 & GRAS family transcription factor & 35.4 & 32.1 & 33.5 & 17.4 & 23.3 & 2.03 & $1.12 \mathrm{E}-06$ \\
\hline AT3G17185 & TASIR-ARF, TAS3, ATTAS3 & 29.0 & 28.6 & 28.0 & 14.4 & 17.5 & 2.02 & 0.000241 \\
\hline AT2G22120 & RING/FYVE/PHD zinc finger superfamily & 23.5 & 17.1 & 15.7 & 11.6 & 24.9 & 2.02 & 0.008653 \\
\hline AT5G65890 & ACR1 & 51.9 & 50.7 & 30.4 & 25.8 & 29.5 & 2.01 & 0.02437 \\
\hline AT2G19500 & CKX2, ATCKX2 & 20.5 & 20.4 & 18.8 & 10.2 & 12.6 & 2.00 & 0.000307 \\
\hline AT2G29490 & ATGSTU1, GST19, GSTU1 & 51.0 & 53.3 & 54.6 & 25.5 & 36.0 & 2.00 & 0.001171 \\
\hline AT3G49120 & ATPERX34, PERX34, PRXCB, ATPCB, PRX34 & 147.2 & 183.5 & 156.4 & 73.6 & 56.1 & 2.00 & $7.45 \mathrm{E}-10$ \\
\hline AT3G47780 & ATATH6, ATH6 & 66.5 & 75.3 & 67.2 & 33.3 & 30.7 & 2.00 & $9.5 \mathrm{E}-05$ \\
\hline AT4G17030 & ATEXLB1 & 61.9 & 65.4 & 50.6 & 31.0 & 41.6 & 2.00 & $9.97 \mathrm{E}-05$ \\
\hline AT5G24850 & CRY3 & 34.8 & 32.0 & 25.4 & 17.4 & 27.7 & 1.99 & $2.13 \mathrm{E}-05$ \\
\hline AT2G05380 & GRP3S & 3277.2 & 2702.5 & 3212.5 & 1650.1 & 2398.4 & 1.99 & $5.1 \mathrm{E}-08$ \\
\hline AT4G28040 & nodulin MtN21 /EamA-like transporter family & 48.8 & 57.8 & 41.9 & 24.7 & 27.2 & 1.97 & 0.000463 \\
\hline AT2G21187 & other RNA & 12.3 & 14.3 & 11.8 & 6.3 & 11.8 & 1.96 & 0.005592 \\
\hline AT1G14520 & MIOX1 & 10.5 & 8.1 & 11.1 & 5.3 & 6.7 & 1.96 & 0.001802 \\
\hline AT1G51070 & bHLH115 & 141.0 & 150.7 & 123.5 & 72.0 & 83.1 & 1.96 & $3.2 \mathrm{E}-07$ \\
\hline AT5G47220 & ATERF2, ATERF-2, ERF2 & 23.9 & 23.0 & 23.3 & 12.3 & 17.6 & 1.94 & $3.82 \mathrm{E}-05$ \\
\hline AT1G05570 & CALS1 & 167.2 & 186.6 & 156.0 & 86.1 & 95.2 & 1.94 & $8.46 \mathrm{E}-08$ \\
\hline AT1G29640 & Protein of unknown function, DUF584 & 41.8 & 40.0 & 38.8 & 21.6 & 31.6 & 1.94 & $6.39 \mathrm{E}-05$ \\
\hline AT3G55880 & Alpha/beta hydrolase related protein & 131.8 & 163.3 & 128.8 & 68.0 & 83.5 & 1.94 & $4.51 \mathrm{E}-05$ \\
\hline AT3G46130 & ATMYB48-1 & 61.0 & 56.5 & 57.3 & 31.6 & 39.9 & 1.93 & $8.45 \mathrm{E}-05$ \\
\hline AT5G48010 & THAS, THAS1 & 210.4 & 331.5 & 217.9 & 109.0 & 49.3 & 1.93 & 0.000299 \\
\hline AT2G36950 & $\begin{array}{l}\text { Heavy metal transport/detoxification } \\
\text { superfamily protein }\end{array}$ & 191.6 & 194.8 & 179.2 & 99.5 & 137.6 & 1.93 & $3.14 \mathrm{E}-06$ \\
\hline AT2G15480 & UGT73B5 & 139.6 & 134.1 & 136.3 & 72.5 & 117.0 & 1.92 & $8.91 \mathrm{E}-05$ \\
\hline AT4G15760 & MO1 & 201.3 & 184.5 & 258.9 & 104.7 & 155.8 & 1.92 & $2.39 \mathrm{E}-05$ \\
\hline AT2G06005 & FIP1 & 69.4 & 48.3 & 63.9 & 36.1 & 42.4 & 1.92 & 0.028667 \\
\hline AT1G79410 & AtOCT5, 5-Oct & 173.4 & 167.3 & 161.9 & 90.3 & 121.1 & 1.92 & $3.45 \mathrm{E}-07$ \\
\hline AT2G16660 & Major facilitator superfamily protein & 486.4 & 398.1 & 494.9 & 254.2 & 333.9 & 1.91 & 0.000395 \\
\hline AT4G16260 & Glycosyl hydrolase superfamily protein & 266.5 & 379.0 & 267.8 & 139.6 & 128.7 & 1.91 & $4.8 \mathrm{E}-06$ \\
\hline AT1G14540 & Peroxidase superfamily protein & 56.3 & 66.0 & 57.8 & 29.7 & 27.2 & 1.90 & $6.26 \mathrm{E}-06$ \\
\hline AT3G14690 & CYP72A15 & 33.2 & 37.5 & 39.4 & 17.5 & 25.7 & 1.90 & $2.61 \mathrm{E}-05$ \\
\hline AT5G49990 & Xanthine/uracil permease family protein & 131.0 & 127.6 & 102.9 & 69.0 & 77.6 & 1.90 & $1.73 \mathrm{E}-06$ \\
\hline AT3G13062 & $\begin{array}{l}\text { Polyketide cyclase/dehydrase and lipid } \\
\text { transport superfamily protein }\end{array}$ & 25.0 & 18.4 & 19.8 & 13.2 & 16.7 & 1.89 & 0.009946 \\
\hline AT1G07860 & $\begin{array}{l}\text { BEST Arabidopsis thaliana protein match is: } \\
\text { Protein kinase superfamily protein }\end{array}$ & 7.0 & 5.1 & 4.8 & 3.7 & 5.0 & 1.89 & 0.013087 \\
\hline AT2G18230 & AtPPa2, PPa2 & 426.5 & 531.2 & 362.6 & 226.5 & 298.0 & 1.88 & 0.002533 \\
\hline AT1G30820 & CTP synthase family protein & 453.8 & 319.9 & 467.2 & 241.6 & 276.6 & 1.88 & $1.92 \mathrm{E}-08$ \\
\hline AT1G33110 & MATE efflux family protein & 129.3 & 145.6 & 148.9 & 68.9 & 108.3 & 1.88 & $3.94 \mathrm{E}-05$ \\
\hline AT5G66930 & unknown protein & 8.0 & 6.2 & 6.0 & 4.3 & 5.7 & 1.88 & 0.027026 \\
\hline AT1G69260 & AFP1 & 72.7 & 102.7 & 60.9 & 38.8 & 44.5 & 1.87 & 0.004775 \\
\hline AT4G19880 & Glutathione S-transferase family protein & 466.5 & 432.5 & 524.1 & 249.5 & 317.3 & 1.87 & $3.18 \mathrm{E}-06$ \\
\hline AT1G72680 & ATCAD1, CAD1 & 263.9 & 233.9 & 287.3 & 141.8 & 182.2 & 1.86 & $2.27 \mathrm{E}-06$ \\
\hline AT3G46130 & MYB48 & 132.3 & 147.7 & 116.0 & 71.5 & 91.3 & 1.85 & 0.006585 \\
\hline AT4G33040 & Thioredoxin superfamily protein & 9.3 & 10.7 & 7.4 & 5.0 & 7.1 & 1.85 & 0.000282 \\
\hline AT5G61960 & AML1, ML1 & 51.4 & 34.7 & 30.3 & 27.9 & 48.4 & 1.84 & 0.049043 \\
\hline $5 G 43270$ & SPL2 & 28.5 & 18.5 & 28.3 & 15.5 & 25.2 & 1.84 & 0.0087 \\
\hline
\end{tabular}




\begin{tabular}{|c|c|c|c|c|c|c|c|c|}
\hline AT1G51690 & ATB ALPHA, B ALPHA & 9.0 & 8.1 & 5.6 & 4.9 & 4.7 & 1.84 & 0.045732 \\
\hline AT3G63280 & ATNEK4, NEK4 & 38.1 & 28.7 & 23.6 & 20.8 & 25.6 & 1.84 & 0.040767 \\
\hline AT1G54740 & Protein of unknown function (DUF3049) & 22.7 & 20.7 & 23.0 & 12.4 & 15.8 & 1.83 & $1.01 \mathrm{E}-06$ \\
\hline AT4G19660 & NPR4, ATNPR4 & 105.0 & 106.7 & 98.5 & 57.4 & 75.3 & 1.83 & $6.51 \mathrm{E}-06$ \\
\hline AT3G62400 & unknown protein & 42.8 & 42.9 & 42.0 & 23.5 & 29.9 & 1.82 & 0.029694 \\
\hline AT3G44310 & NIT1, ATNIT1, NITI & 207.4 & 187.1 & 175.9 & 114.2 & 136.9 & 1.82 & $4.75 \mathrm{E}-06$ \\
\hline AT2G43120 & RmlC-like cupins superfamily protein & 16.0 & 19.7 & 14.2 & 8.8 & 10.7 & 1.81 & $1.12 \mathrm{E}-05$ \\
\hline AT5G49015 & Expressed protein & 22.7 & 16.5 & 19.3 & 12.6 & 14.8 & 1.81 & 0.021368 \\
\hline AT4G28040 & nodulin MtN21 /EamA-like transporter family & 5.4 & 4.4 & 4.3 & 3.0 & 3.8 & 1.81 & 0.037935 \\
\hline AT5G65445 & pre-tRNA & 8.4 & 9.3 & 6.9 & 4.7 & 6.8 & 1.81 & 0.004116 \\
\hline AT2G16700 & ADF5, ATADF5 & 8.4 & 5.7 & 6.0 & 4.7 & 9.8 & 1.80 & 0.001784 \\
\hline AT4G34135 & UGT73B2 & 107.6 & 125.0 & 141.3 & 59.7 & 89.8 & 1.80 & 0.000146 \\
\hline AT3G51895 & SULTR3 & 174.7 & 270.0 & 182.2 & 96.9 & 124.7 & 1.80 & 0.035291 \\
\hline AT1G54100 & ALDH7B4 & 463.5 & 463.0 & 504.5 & 257.3 & 300.2 & 1.80 & 0.000101 \\
\hline AT3G21260 & Glycolipid transfer protein (GLTP) family & 55.2 & 56.6 & 55.4 & 30.7 & 40.5 & 1.80 & $3.23 \mathrm{E}-05$ \\
\hline AT3G51430 & YLS2, SSL5 & 122.0 & 98.5 & 142.8 & 68.1 & 97.4 & 1.79 & 0.010375 \\
\hline AT3G14680 & CYP72A14 & 64.7 & 81.9 & 69.8 & 36.1 & 23.4 & 1.79 & 0.000108 \\
\hline AT2G30140 & UDP-Glycosyltransferase superfamily protein & 1139.6 & 1041.5 & 1245.8 & 636.4 & 775.8 & 1.79 & $3.23 \mathrm{E}-05$ \\
\hline AT5G57655 & xylose isomerase family protein & 690.7 & 653.2 & 711.3 & 387.3 & 493.8 & 1.78 & $1.04 \mathrm{E}-05$ \\
\hline AT3G19230 & Leucine-rich repeat (LRR) family protein & 43.1 & 35.4 & 35.4 & 24.2 & 30.8 & 1.78 & 4.47E-05 \\
\hline AT2G43820 & GT, UGT74F2, ATSAGT1, SGT1, SAGT1 & 809.9 & 747.6 & 1010.3 & 454.9 & 590.7 & 1.78 & 0.000183 \\
\hline AT3G43430 & RING/U-box superfamily protein & 34.7 & 27.8 & 33.0 & 19.5 & 21.0 & 1.78 & $1 \mathrm{E}-07$ \\
\hline AT1G09500 & NAD(P)-binding Rossmann-fold superfamily & 39.2 & 37.0 & 36.2 & 22.1 & 29.5 & 1.77 & 0.020212 \\
\hline AT4G30490 & AFG1-like ATPase family protein & 251.2 & 286.2 & 236.4 & 142.0 & 181.5 & 1.77 & $2.91 \mathrm{E}-08$ \\
\hline AT5G16140 & Peptidyl-tRNA hydrolase family protein & 7.5 & 6.3 & 5.8 & 4.2 & 4.1 & 1.77 & 0.030192 \\
\hline AT4G36880 & CP1 & 20.8 & 25.1 & 19.0 & 11.8 & 14.0 & 1.76 & 0.000242 \\
\hline AT2G30750 & CYP71A12 & 6.2 & 10.3 & 6.8 & 3.5 & 3.7 & 1.76 & 0.002292 \\
\hline AT5G21280 & hydroxyproline-rich glycoprotein family & 31.6 & 26.1 & 27.1 & 18.0 & 21.9 & 1.76 & $3.8 \mathrm{E}-05$ \\
\hline AT4G15260 & UDP-Glycosyltransferase superfamily protein & 20.0 & 18.9 & 24.9 & 11.4 & 16.4 & 1.76 & 0.000101 \\
\hline AT5G22970 & unknown protein & 5.9 & 5.1 & 4.8 & 3.3 & 5.0 & 1.75 & 0.006324 \\
\hline AT3G26690 & ATNUDT13, ATNUDX13, NUDX13 & 61.7 & 53.9 & 55.3 & 35.3 & 40.0 & 1.75 & 0.000143 \\
\hline AT3G01420 & ALPHA-DOX1, DOX1, DIOX1, PADOX-1 & 997.9 & 1430.4 & 1053.4 & 571.2 & 469.8 & 1.75 & $1.88 \mathrm{E}-05$ \\
\hline AT3G14620 & CYP72A8 & 460.9 & 461.5 & 492.5 & 263.8 & 318.8 & 1.75 & $2.24 \mathrm{E}-06$ \\
\hline AT4G01070 & GT72B1 & 48.1 & 59.4 & 47.3 & 27.6 & 30.3 & 1.75 & $4.86 \mathrm{E}-08$ \\
\hline AT3G44310 & NIT1, ATNIT1, NITI & 1328.2 & 1310.8 & 1155.3 & 762.4 & 907.8 & 1.74 & $1.26 \mathrm{E}-08$ \\
\hline AT1G68568 & other RNA & 107.4 & 116.2 & 77.6 & 61.7 & 73.0 & 1.74 & 0.005516 \\
\hline AT5G27600 & LACS7, ATLACS7 & 174.4 & 172.0 & 183.3 & 100.2 & 132.2 & 1.74 & 5.97E-06 \\
\hline AT3G22410 & $\begin{array}{l}\text { Sec14p-like phosphatidylinositol transfer } \\
\text { family protein }\end{array}$ & 38.4 & 35.0 & 34.6 & 22.1 & 23.8 & 1.74 & 0.000316 \\
\hline AT1G63650 & EGL3, EGL1, ATMYC-2 & 6.3 & 4.5 & 5.5 & 3.6 & 4.1 & 1.74 & 0.014773 \\
\hline AT1G77120 & ADH1, ADH, ATADH, ATADH1 & 120.1 & 170.2 & 133.8 & 69.1 & 95.9 & 1.74 & 0.027218 \\
\hline AT5G37970 & $\begin{array}{l}\text { S-adenosyl-L-methionine-dependent } \\
\text { methyltransferases superfamily protein }\end{array}$ & 13.0 & 9.7 & 8.8 & 7.5 & 8.5 & 1.73 & 0.005814 \\
\hline AT4G26810 & SWIB/MDM2 domain superfamily protein & 29.3 & 25.6 & 30.9 & 16.9 & 19.1 & 1.73 & 0.014454 \\
\hline AT4G29740 & CKX4, ATCKX4 & 75.4 & 77.1 & 76.0 & 43.6 & 36.2 & 1.73 & $5.35 \mathrm{E}-05$ \\
\hline AT4G10400 & F-box/RNI-like/FBD-like domains-containing & 7.8 & 6.2 & 5.3 & 4.5 & 5.5 & 1.73 & 0.000513 \\
\hline AT5G25110 & CIPK25, SnRK3.25 & 8.0 & 11.8 & 7.9 & 4.6 & 5.3 & 1.73 & 0.007518 \\
\hline AT2G31750 & UGT74D1 & 94.1 & 79.3 & 87.2 & 54.6 & 76.3 & 1.73 & $2.11 \mathrm{E}-06$ \\
\hline AT4G21910 & MATE efflux family protein & 9.3 & 5.8 & 7.2 & 5.4 & 8.4 & 1.72 & 0.04954 \\
\hline AT1G21460 & SWEET1, AtSWEET1 & 29.5 & 34.8 & 32.5 & 17.1 & 21.2 & 1.72 & 0.00315 \\
\hline AT2G45023 & other RNA & 27.3 & 27.3 & 24.9 & 15.9 & 26.8 & 1.72 & 0.009741 \\
\hline AT5G67240 & SDN3 & 169.6 & 149.1 & 123.4 & 98.5 & 154.4 & 1.72 & 0.003003 \\
\hline AT5G40850 & UPM1 & 626.1 & 605.0 & 667.7 & 363.9 & 440.7 & 1.72 & $1.82 \mathrm{E}-05$ \\
\hline AT1G80530 & Major facilitator superfamily protein & 85.6 & 91.4 & 80.9 & 49.9 & 68.9 & 1.72 & $2.51 \mathrm{E}-06$ \\
\hline AT5G19440 & NAD(P)-binding Rossmann-fold superfamily & 542.3 & 539.6 & 601.5 & 316.4 & 352.1 & 1.71 & 0.000108 \\
\hline AT3G07730 & unknown protein & 27.9 & 36.8 & 23.8 & 16.3 & 20.0 & 1.71 & 0.029689 \\
\hline AT3G56880 & VQ motif-containing protein & 144.6 & 168.7 & 168.9 & 84.5 & 112.6 & 1.71 & $5.42 \mathrm{E}-05$ \\
\hline AT4G10510 & Subtilase family protein & 40.6 & 30.2 & 40.1 & 23.7 & 20.1 & 1.71 & 0.00111 \\
\hline AT2G01890 & PAP8, ATPAP8 & 180.0 & 183.8 & 168.7 & 105.4 & 99.9 & 1.71 & 0.000207 \\
\hline AT2G15220 & Plant basic secretory protein (BSP) family & 113.6 & 181.8 & 151.8 & 66.6 & 71.6 & 1.71 & 0.003568 \\
\hline AT5G11950 & Putative lysine decarboxylase family protein & 156.3 & 153.1 & 122.4 & 91.6 & 147.4 & 1.71 & 0.007659 \\
\hline AT1G05562 & $\begin{array}{l}\text { Potential natural antisense gene, locus } \\
\text { overlaps with AT1G05560 }\end{array}$ & 128.5 & 127.4 & 128.0 & 75.4 & 94.4 & 1.70 & 1.79E-06 \\
\hline AT2G44300 & $\begin{array}{l}\text { Bifunctional inhibitor/lipid-transfer } \\
\text { protein/seed storage } 2 \mathrm{~S} \text { albumin superfamily }\end{array}$ & 22.7 & 22.6 & 20.2 & 13.3 & 17.2 & 1.70 & 0.001176 \\
\hline AT5G17000 & Zinc-binding dehydrogenase family protein & 8.6 & 7.9 & 7.3 & 5.0 & 6.6 & 1.70 & 0.00077 \\
\hline AT1G24400 & LHT2, AATL2, ATLHT2 & 105.9 & 104.1 & 95.5 & 62.4 & 82.6 & 1.70 & $2.84 \mathrm{E}-06$ \\
\hline AT2G32170 & $\begin{array}{l}\text { S-adenosyl-L-methionine-dependent } \\
\text { methyltransferases superfamily protein }\end{array}$ & 77.4 & 57.5 & 51.1 & 45.6 & 58.2 & 1.70 & 0.027892 \\
\hline AT1G65970 & TPX2 & 12.7 & 13.3 & 11.4 & 7.5 & 6.5 & 1.70 & 0.004676 \\
\hline AT5G45080 & AtPP2-A6, PP2-A6 & 42.9 & 49.4 & 46.8 & 25.3 & 20.6 & 1.70 & $7.72 \mathrm{E}-05$ \\
\hline AT3G57520 & AtSIP2, SIP2 & 862.5 & 576.9 & 927.6 & 510.3 & 556.1 & 1.69 & 0.00077 \\
\hline AT1G05700 & $\begin{array}{l}\text { Leucine-rich repeat transmembrane protein } \\
\text { kinase protein }\end{array}$ & 19.7 & 25.2 & 17.6 & 11.7 & 9.8 & 1.69 & 0.000129 \\
\hline AT4G22070 & WRKY31, ATWRKY31 & 128.9 & 157.3 & 125.1 & 76.4 & 63.1 & 1.69 & 0.00012 \\
\hline AT4G33150 & $\begin{array}{l}\text { lysine-ketoglutarate reductase/saccharopine } \\
\text { dehydrogenase bifunctional enzyme }\end{array}$ & 163.6 & 234.3 & 158.4 & 97.0 & 87.6 & 1.69 & 0.00038 \\
\hline AT1G30410 & ATMRP13, MRP13, ABCC12 & 55.6 & 56.7 & 64.4 & 32.9 & 43.1 & 1.69 & 0.003589 \\
\hline AT1G04350 & $\begin{array}{l}\text { 2-oxoglutarate (2OG) and Fe(II)-dependent } \\
\text { oxygenase superfamily protein }\end{array}$ & 72.9 & 76.8 & 69.0 & 43.3 & 48.2 & 1.68 & $1.3 \mathrm{E}-05$ \\
\hline AT5G15860 & ATPCME, PCME & 43.2 & 47.5 & 41.0 & 25.7 & 34.2 & 1.68 & 0.000138 \\
\hline
\end{tabular}




\begin{tabular}{|c|c|c|c|c|c|c|c|c|}
\hline AT4G21990 & APR3 & 1177.5 & 1009.1 & 1341.2 & 700.4 & 1019.2 & 1.68 & 0.000555 \\
\hline AT1G79170 & unknown protein & 48.2 & 37.4 & 33.6 & 28.7 & 32.8 & 1.68 & 0.003705 \\
\hline AT1G14860 & atnudt18, NUDT18 & 64.8 & 64.9 & 64.5 & 38.6 & 36.2 & 1.68 & $4.28 \mathrm{E}-06$ \\
\hline AT3G22200 & POP2 & 1022.3 & 949.4 & 1039.5 & 608.8 & 688.5 & 1.68 & $1.43 \mathrm{E}-07$ \\
\hline AT1G30700 & FAD-binding Berberine family protein & 69.4 & 84.8 & 75.4 & 41.4 & 30.5 & 1.68 & 0.000433 \\
\hline AT1G67980 & CCOAMT & 10.0 & 10.5 & 11.3 & 6.0 & 5.7 & 1.67 & 0.031105 \\
\hline AT3G52060 & $\begin{array}{l}\text { Core-2/I-branching beta-1,6-N- } \\
\text { acetylglucosaminyltransferase family protein }\end{array}$ & 83.8 & 98.7 & 102.2 & 50.3 & 71.3 & 1.67 & $2.38 \mathrm{E}-05$ \\
\hline AT1G58590 & other RNA & 31.9 & 36.1 & 31.7 & 19.2 & 22.1 & 1.67 & 0.000402 \\
\hline AT5G12323 & unknown protein & 8.6 & 7.5 & 7.1 & 5.2 & 5.8 & 1.67 & 0.014078 \\
\hline AT3G14990 & $\begin{array}{l}\text { Class I glutamine amidotransferase-like } \\
\text { superfamily protein }\end{array}$ & 1151.0 & 902.7 & 1161.8 & 691.6 & 956.4 & 1.66 & $5.06 \mathrm{E}-07$ \\
\hline AT1G17250 & AtRLP3, RLP3 & 38.6 & 29.0 & 36.7 & 23.2 & 31.1 & 1.66 & 0.003393 \\
\hline AT2G20650 & RING/U-box superfamily protein & 5.9 & 5.9 & 4.4 & 3.6 & 5.8 & 1.66 & 0.020359 \\
\hline AT1G50590 & RmlC-like cupins superfamily protein & 11.9 & 12.8 & 11.0 & 7.2 & 8.2 & 1.66 & 0.000172 \\
\hline AT2G23910 & NAD(P)-binding Rossmann-fold superfamily & 143.5 & 109.0 & 116.6 & 86.7 & 91.2 & 1.66 & 0.000453 \\
\hline AT1G72770 & HAB1 & 47.2 & 50.7 & 42.7 & 28.5 & 33.1 & 1.65 & 0.001558 \\
\hline AT5G63530 & ATFP3, FP3 & 182.7 & 219.2 & 157.0 & 110.5 & 159.5 & 1.65 & 0.000498 \\
\hline AT1G15960 & NRAMP6, ATNRAMP6 & 20.1 & 19.2 & 14.1 & 12.1 & 17.3 & 1.65 & 0.005585 \\
\hline AT3G57520 & AtSIP2, SIP2 & 1355.3 & 913.9 & 1362.6 & 821.4 & 970.6 & 1.65 & 0.000208 \\
\hline AT5G65980 & Auxin efflux carrier family protein & 49.7 & 48.1 & 64.4 & 30.2 & 23.3 & 1.65 & 0.000476 \\
\hline AT2G33480 & ANAC041, NAC041 & 94.0 & 90.5 & 82.6 & 57.1 & 81.7 & 1.64 & 0.000161 \\
\hline AT5G13370 & Auxin-responsive GH3 family protein & 405.8 & 378.5 & 390.0 & 246.9 & 304.1 & 1.64 & $6.95 \mathrm{E}-06$ \\
\hline AT3G49780 & ATPSK4, ATPSK3 (FORMER SYMBOL), PSK4 & 229.5 & 204.8 & 236.2 & 139.9 & 136.8 & 1.64 & 0.00011 \\
\hline AT2G15490 & UGT73B4 & 93.6 & 88.1 & 112.2 & 57.1 & 66.8 & 1.64 & 0.003845 \\
\hline AT2G29420 & ATGSTU7, GST25, GSTU7 & 55.3 & 54.4 & 54.1 & 33.8 & 45.5 & 1.64 & $7.02 \mathrm{E}-05$ \\
\hline AT2G29500 & HSP20-like chaperones superfamily protein & 25.3 & 27.0 & 23.6 & 15.5 & 18.7 & 1.63 & 0.001711 \\
\hline AT1G20880 & RNA-binding (RRM/RBD/RNP motifs) family & 42.7 & 37.1 & 40.5 & 26.2 & 37.1 & 1.63 & 0.000563 \\
\hline AT5G65380 & MATE efflux family protein & 457.2 & 492.4 & 472.2 & 280.5 & 258.0 & 1.63 & 0.000136 \\
\hline AT4G21990 & APR3, PRH-26, PRH26, ATAPR3 & 961.1 & 755.9 & 1032.0 & 590.5 & 755.1 & 1.63 & 0.00147 \\
\hline AT4G37980 & ELI3-1, ELI3, ATCAD7, CAD7 & 60.0 & 63.7 & 46.9 & 37.0 & 52.6 & 1.62 & 0.002785 \\
\hline AT4G23050 & $\begin{array}{l}\text { PAS domain-containing protein tyrosine } \\
\text { kinase family protein }\end{array}$ & 68.7 & 87.6 & 67.7 & 42.4 & 45.6 & 1.62 & 2.97E-05 \\
\hline AT5G06750 & Protein phosphatase $2 \mathrm{C}$ family protein & 64.3 & 62.4 & 52.9 & 39.8 & 41.3 & 1.62 & $9.66 \mathrm{E}-05$ \\
\hline AT2G46270 & GBF3 & 188.9 & 231.0 & 154.6 & 116.7 & 134.4 & 1.62 & 0.008365 \\
\hline AT1G64950 & CYP89A5 & 32.0 & 34.1 & 36.5 & 19.8 & 21.7 & 1.62 & 0.002303 \\
\hline AT1G12050 & fumarylacetoacetase, putative & 347.5 & 341.3 & 343.1 & 215.0 & 261.3 & 1.62 & $1.17 \mathrm{E}-05$ \\
\hline AT1G65690 & $\begin{array}{l}\text { Late embryogenesis abundant (LEA) } \\
\text { hydroxyproline-rich glycoprotein family }\end{array}$ & 24.3 & 32.3 & 23.5 & 15.0 & 17.6 & 1.61 & 0.000722 \\
\hline AT4G15490 & UGT84A3 & 60.7 & 59.7 & 66.0 & 37.6 & 44.8 & 1.61 & $4.03 \mathrm{E}-05$ \\
\hline AT1G18970 & GLP4 & 12.9 & 15.8 & 15.3 & 8.0 & 7.1 & 1.61 & 0.009147 \\
\hline AT1G73390 & $\begin{array}{l}\text { Endosomal targeting BRO1-like domain- } \\
\text { containing protein }\end{array}$ & 145.0 & 164.0 & 129.0 & 90.2 & 106.4 & 1.61 & 0.002587 \\
\hline AT5G26900 & $\begin{array}{l}\text { Transducin family protein / WD- } 40 \text { repeat } \\
\text { family protein }\end{array}$ & 10.6 & 11.6 & 8.7 & 6.6 & 11.2 & 1.61 & 0.008029 \\
\hline AT4G32010 & HSL1, HSI2-L1, VAL2 & 185.1 & 168.7 & 176.1 & 115.5 & 138.5 & 1.60 & $2.84 \mathrm{E}-07$ \\
\hline AT2G38880 & NF-YB1 & 6.0 & 4.2 & 4.8 & 3.7 & 3.5 & 1.60 & 0.036985 \\
\hline AT3G57040 & ARR9, ATRR4 & 65.7 & 67.7 & 55.9 & 41.1 & 48.0 & 1.60 & 0.006834 \\
\hline AT1G29200 & O-fucosyltransferase family protein & 5.0 & 4.0 & 3.5 & 3.1 & 5.0 & 1.59 & 0.041085 \\
\hline AT4G34138 & UGT73B1 & 510.1 & 387.0 & 483.1 & 319.9 & 404.4 & 1.59 & $9.64 \mathrm{E}-05$ \\
\hline AT5G44005 & unknown protein & 32.9 & 31.1 & 33.8 & 20.6 & 26.3 & 1.59 & 0.000784 \\
\hline AT3G12520 & SULTR4 & 78.1 & 73.8 & 63.7 & 49.0 & 41.1 & 1.59 & 0.040093 \\
\hline AT3G03300 & DCL2 & 65.4 & 71.1 & 63.7 & 41.0 & 55.7 & 1.59 & 0.001375 \\
\hline AT1G08230 & $\begin{array}{l}\text { Transmembrane amino acid transporter } \\
\text { family protein }\end{array}$ & 133.8 & 141.0 & 107.5 & 84.0 & 94.2 & 1.59 & $5.51 \mathrm{E}-06$ \\
\hline AT5G14780 & $\mathrm{FDH}$ & 1450.5 & 1519.0 & 1550.3 & 911.1 & 1088.1 & 1.59 & 0.000144 \\
\hline AT5G56470 & $\begin{array}{l}\text { FAD-dependent oxidoreductase family } \\
\text { protein }\end{array}$ & 18.3 & 17.0 & 14.4 & 11.5 & 14.2 & 1.59 & 0.029523 \\
\hline AT4G28870 & unknown protein & 287.9 & 233.6 & 214.5 & 181.0 & 180.8 & 1.59 & 0.002567 \\
\hline AT1G03220 & Eukaryotic aspartyl protease family protein & 117.9 & 129.3 & 132.8 & 74.2 & 77.3 & 1.59 & 0.000122 \\
\hline AT1G23760 & JP630, PG3 & 49.0 & 42.6 & 43.2 & 30.8 & 28.7 & 1.59 & $2 \mathrm{E}-06$ \\
\hline AT5G64250 & Aldolase-type TIM barrel family protein & 163.8 & 185.0 & 149.5 & 103.3 & 122.4 & 1.59 & $2.25 \mathrm{E}-05$ \\
\hline AT2G01422 & $\begin{array}{l}\text { chr2:183940-184734 FORWARD } \\
\text { LENGTH=795 }\end{array}$ & 6.1 & 6.3 & 4.3 & 3.9 & 4.5 & 1.58 & 0.002634 \\
\hline AT5G16120 & alpha/beta-Hydrolases superfamily protein & 19.4 & 15.4 & 18.8 & 12.3 & 14.7 & 1.58 & 0.042856 \\
\hline AT1G66200 & ATGSR2, GSR2, GLN1 & 658.5 & 781.9 & 768.5 & 416.5 & 361.9 & 1.58 & 0.001236 \\
\hline AT5G62480 & ATGSTU9, GST14, GST14B, GSTU9 & 63.6 & 76.9 & 70.5 & 40.2 & 34.3 & 1.58 & 0.00375 \\
\hline AT3G21260 & GLTP3 & 65.6 & 66.1 & 46.9 & 41.5 & 50.6 & 1.58 & 0.030872 \\
\hline AT1G69880 & ATH8, TH8 & 17.6 & 18.4 & 19.0 & 11.2 & 10.3 & 1.58 & 0.009941 \\
\hline AT5G15610 & Proteasome component $(\mathrm{PCl})$ domain protein & 5.8 & 4.0 & 4.4 & 3.7 & 4.5 & 1.58 & 0.017036 \\
\hline AT2G40780 & Nucleic acid-binding, OB-fold-like protein & 16.2 & 14.2 & 15.5 & 10.3 & 10.7 & 1.58 & 0.021475 \\
\hline AT1G74390 & $\begin{array}{l}\text { Polynucleotidyl transferase, ribonuclease } \mathrm{H} \text { - } \\
\text { like superfamily protein }\end{array}$ & 79.6 & 92.9 & 86.0 & 50.6 & 39.9 & 1.57 & 0.004862 \\
\hline AT1G62570 & FMO GS-OX4 & 66.0 & 94.3 & 59.2 & 42.0 & 46.6 & 1.57 & 0.001345 \\
\hline AT3G50740 & UGT72E1 & 226.9 & 260.7 & 236.9 & 144.6 & 123.1 & 1.57 & 0.000233 \\
\hline AT2G34660 & MRP2, ABCC2, AtABCC2 & 279.4 & 259.4 & 273.4 & 178.2 & 209.0 & 1.57 & $5.4 \mathrm{E}-05$ \\
\hline AT1G56145 & $\begin{array}{l}\text { Leucine-rich repeat transmembrane protein } \\
\text { kinase }\end{array}$ & 73.8 & 75.0 & 72.3 & 47.1 & 55.0 & 1.57 & $3.31 \mathrm{E}-06$ \\
\hline AT2G43620 & Chitinase family protein & 74.3 & 75.0 & 63.5 & 47.5 & 62.7 & 1.57 & 0.017508 \\
\hline AT1G26390 & FAD-binding Berberine family protein & 21.8 & 30.2 & 27.6 & 13.9 & 10.2 & 1.57 & 0.007755 \\
\hline AT2G38180 & SGNH hydrolase-type esterase superfamily & 86.6 & 69.1 & 80.7 & 55.3 & 65.5 & 1.57 & . \\
\hline
\end{tabular}




\begin{tabular}{|c|c|c|c|c|c|c|c|c|}
\hline AT1G50055 & TAS1B & 139.3 & 116.9 & 119.2 & 89.2 & 128.2 & 1.56 & 0.002875 \\
\hline AT1G76600 & unknown protein & 31.9 & 28.5 & 35.2 & 20.5 & 30.6 & 1.56 & 0.003443 \\
\hline AT1G60730 & NAD(P)-linked oxidoreductase superfamily & 130.3 & 139.2 & 177.2 & 83.6 & 92.9 & 1.56 & 0.003612 \\
\hline AT1G80290 & $\begin{array}{l}\text { Nucleotide-diphospho-sugar transferases } \\
\text { superfamily protein }\end{array}$ & 30.0 & 21.3 & 20.6 & 19.3 & 21.2 & 1.56 & 0.008097 \\
\hline AT1G49130 & $\begin{array}{l}\text { B-box type zinc finger protein with CCT } \\
\text { domain }\end{array}$ & 18.4 & 19.7 & 23.1 & 11.8 & 17.6 & 1.56 & 0.009306 \\
\hline AT1G28260 & Telomerase activating protein Est1 & 105.1 & 76.1 & 127.8 & 67.6 & 68.0 & 1.55 & 0.010228 \\
\hline AT4G37295 & unknown protein & 18.7 & 13.8 & 15.4 & 12.1 & 17.1 & 1.55 & 0.000489 \\
\hline AT5G20885 & RING/U-box superfamily protein & 89.0 & 111.9 & 92.3 & 57.4 & 70.4 & 1.55 & $4.31 \mathrm{E}-05$ \\
\hline AT2G07140 & $\begin{array}{l}\text { F-box and associated interaction domains- } \\
\text { containing protein }\end{array}$ & 7.2 & 6.0 & 5.1 & 4.7 & 5.2 & 1.55 & 0.000104 \\
\hline AT5G07860 & HXXXD-type acyl-transferase family protein & 14.7 & 12.8 & 14.0 & 9.5 & 11.3 & 1.55 & $3.66 \mathrm{E}-05$ \\
\hline AT5G47560 & ATTDT, ATSDAT, TDT & 536.9 & 594.0 & 602.5 & 347.2 & 336.5 & 1.55 & 0.002085 \\
\hline AT1G54020 & $\begin{array}{l}\text { GDSL-like Lipase/Acylhydrolase superfamily } \\
\text { protein }\end{array}$ & 5.0 & 4.2 & 3.4 & 3.2 & 3.9 & 1.55 & 0.002525 \\
\hline AT1G08220 & FUNCTIONS IN: molecular_function unknown & 193.4 & 164.3 & 168.7 & 125.1 & 116.8 & 1.55 & 0.033143 \\
\hline AT4G34000 & ABF3, DPBF5 & 112.8 & 153.0 & 94.6 & 73.0 & 90.0 & 1.55 & 0.02235 \\
\hline AT1G21000 & PLATZ transcription factor family protein & 129.0 & 115.6 & 136.2 & 83.5 & 93.9 & 1.54 & 0.003908 \\
\hline AT5G35603 & Protein of unknown function (DUF3287) & 20.8 & 16.7 & 17.0 & 13.5 & 13.4 & 1.54 & 0.048617 \\
\hline AT2G30620 & $\begin{array}{l}\text { winged-helix DNA-binding transcription } \\
\text { factor family protein }\end{array}$ & 68.4 & 49.5 & 59.6 & 44.3 & 48.3 & 1.54 & 0.027922 \\
\hline AT5G17010 & Major facilitator superfamily protein & 10.0 & 8.5 & 10.1 & 6.5 & 5.7 & 1.54 & 0.041014 \\
\hline AT1G70790 & $\begin{array}{l}\text { Calcium-dependent lipid-binding (CaLB } \\
\text { domain) family protein }\end{array}$ & 85.8 & 72.0 & 84.8 & 55.6 & 59.0 & 1.54 & 0.00113 \\
\hline AT5G18840 & Major facilitator superfamily protein & 18.0 & 17.5 & 16.3 & 11.7 & 11.6 & 1.54 & 0.004931 \\
\hline AT3G54950 & PLP7, PLA IIIA & 78.6 & 77.1 & 63.6 & 51.0 & 58.7 & 1.54 & $9.16 \mathrm{E}-05$ \\
\hline AT3G51430 & YLS2 & 105.3 & 83.7 & 101.9 & 68.5 & 83.2 & 1.54 & 0.002719 \\
\hline AT3G11930 & $\begin{array}{l}\text { Adenine nucleotide alpha hydrolases-like } \\
\text { superfamily protein }\end{array}$ & 33.4 & 24.4 & 27.1 & 21.7 & 18.9 & 1.54 & 0.021078 \\
\hline AT5G23370 & $\begin{array}{l}\text { GRAM domain-containing protein / ABA- } \\
\text { responsive protein-related }\end{array}$ & 9.6 & 7.6 & 7.3 & 6.2 & 6.6 & 1.53 & 0.00844 \\
\hline AT1G29195 & unknown protein & 35.7 & 40.3 & 40.4 & 23.3 & 25.3 & 1.53 & 0.01857 \\
\hline AT1G24350 & $\begin{array}{l}\text { Acid phosphatase/vanadium-dependent } \\
\text { haloperoxidase-related protein }\end{array}$ & 41.9 & 45.2 & 40.9 & 27.3 & 36.1 & 1.53 & 0.018452 \\
\hline AT4G32175 & PNAS-3 related & 29.9 & 28.0 & 22.4 & 19.5 & 21.4 & 1.53 & 0.008029 \\
\hline AT2G37240 & Thioredoxin superfamily protein & 322.9 & 351.7 & 282.7 & 210.8 & 245.3 & 1.53 & $1.9 \mathrm{E}-06$ \\
\hline AT5G47720 & Thiolase family protein & 84.1 & 78.6 & 75.8 & 54.9 & 67.0 & 1.53 & 0.000585 \\
\hline AT4G16745 & Exostosin family protein & 14.2 & 13.8 & 12.5 & 9.3 & 10.6 & 1.53 & 0.000443 \\
\hline AT2G37750 & unknown protein & 43.1 & 49.1 & 45.4 & 28.2 & 26.3 & 1.53 & 0.004385 \\
\hline AT3G01970 & WRKY45, ATWRKY45 & 84.5 & 83.2 & 89.0 & 55.2 & 55.9 & 1.53 & 0.002944 \\
\hline AT4G33070 & $\begin{array}{l}\text { Thiamine pyrophosphate dependent } \\
\text { pyruvate decarboxylase family protein }\end{array}$ & 70.5 & 98.2 & 87.8 & 46.2 & 47.8 & 1.53 & 0.00356 \\
\hline AT2G02390 & ATGSTZ1, GST18, GSTZ1 & 1094.3 & 1190.9 & 1139.3 & 717.1 & 754.4 & 1.53 & $1.31 \mathrm{E}-05$ \\
\hline AT1G26410 & FAD-binding Berberine family protein & 8.9 & 11.1 & 8.9 & 5.8 & 4.9 & 1.53 & 0.034838 \\
\hline AT3G14020 & NF-YA6 & 140.8 & 131.0 & 146.7 & 92.3 & 122.9 & 1.53 & $9.18 \mathrm{E}-05$ \\
\hline AT3G12955 & SAUR-like auxin-responsive protein family & 25.1 & 20.3 & 19.7 & 16.5 & 19.3 & 1.53 & 0.000177 \\
\hline AT1G70530 & CRK3 & 141.0 & 146.6 & 147.9 & 92.6 & 129.8 & 1.52 & 0.000719 \\
\hline AT5G47700 & $60 \mathrm{~S}$ acidic ribosomal protein family & 5.4 & 4.5 & 4.6 & 3.6 & 3.8 & 1.52 & 0.002895 \\
\hline AT1G78380 & ATGSTU19, GST8, GSTU19 & 682.8 & 758.1 & 707.6 & 449.1 & 589.1 & 1.52 & 0.001484 \\
\hline AT1G09420 & G6PD4 & 38.7 & 36.6 & 34.2 & 25.5 & 30.4 & 1.52 & $9.58 \mathrm{E}-05$ \\
\hline AT2G35940 & BLH1 & 577.8 & 629.7 & 582.2 & 380.6 & 451.4 & 1.52 & 0.002556 \\
\hline AT1G14130 & $\begin{array}{l}\text { 2-oxoglutarate (2OG) and Fe(II)-dependent } \\
\text { oxygenase superfamily protein }\end{array}$ & 12.0 & 11.1 & 11.0 & 7.9 & 9.4 & 1.52 & 0.002771 \\
\hline AT3G07960 & $\begin{array}{l}\text { Phosphatidylinositol-4-phosphate 5-kinase } \\
\text { family protein }\end{array}$ & 13.9 & 12.7 & 13.0 & 9.2 & 11.5 & 1.52 & 0.00282 \\
\hline AT4G30470 & NAD(P)-binding Rossmann-fold superfamily & 123.6 & 111.3 & 107.3 & 81.5 & 86.7 & 1.52 & 0.001267 \\
\hline AT5G28780 & PIF1 helicase & 10.1 & 7.7 & 7.4 & 6.7 & 8.1 & 1.52 & 0.017381 \\
\hline AT5G45870 & PYL12, RCAR6 & 7.7 & 5.6 & 6.7 & 5.1 & 5.6 & 1.52 & 0.02092 \\
\hline AT1G04380 & $\begin{array}{l}\text { 2-oxoglutarate (2OG) and Fe(II)-dependent } \\
\text { oxygenase superfamily protein }\end{array}$ & 7.2 & 6.3 & 5.1 & 4.8 & 5.2 & 1.51 & 0.00124 \\
\hline AT2G07680 & ATMRP11, MRP11, ABCC13 & 41.2 & 35.9 & 32.1 & 27.3 & 34.9 & 1.51 & 0.000644 \\
\hline AT3G54680 & proteophosphoglycan-related & 201.4 & 220.5 & 186.3 & 133.1 & 148.3 & 1.51 & 0.003763 \\
\hline AT1G33060 & ANAC014, NAC014 & 44.9 & 43.7 & 43.5 & 29.7 & 39.5 & 1.51 & 0.000397 \\
\hline AT4G10380 & NIP5 & 58.3 & 57.4 & 51.5 & 38.6 & 41.4 & 1.51 & 0.004553 \\
\hline
\end{tabular}


Table S2 List of top 100 genes that are up- or down-regulated in roxy19DS plants ( $F C>1.5, p<0.05)$.

\begin{tabular}{|c|c|c|c|c|c|c|c|c|}
\hline \multicolumn{9}{|c|}{ Genes that are up-regulated in roxy19DS plants $(F C>1.5, p<0.05)$} \\
\hline \multirow{2}{*}{ AGI code } & \multirow{2}{*}{ Description } & \multicolumn{5}{|c|}{ mean of linear expression values } & \multirow{2}{*}{$\begin{array}{l}\text { Nossen/ } \\
\text { roxy19DS }\end{array}$} & \multirow{2}{*}{$p$-value } \\
\hline & & Col-0 & CCMC\#8 & CCMC\#12 & Nossen & roxy19DS & & \\
\hline AT1G67105 & other RNA & 19.8 & 13.8 & 17.0 & 31.3 & 899.0 & 28.72 & $2.78 \mathrm{E}-10$ \\
\hline AT3G48560 & CSR1, ALS, AHAS, TZP5, IMR1 & 225.7 & 232.0 & 221.2 & 215.0 & 1668.3 & 7.76 & $4.1 \mathrm{E}-10$ \\
\hline AT1G62250 & unknown protein & 11.4 & 18.0 & 22.0 & 9.1 & 41.9 & 4.60 & 0.006234 \\
\hline AT4G21650 & Subtilase family protein & 434.3 & 382.2 & 381.9 & 315.5 & 1345.3 & 4.26 & $7.34 \mathrm{E}-07$ \\
\hline AT2G07689 & $\begin{array}{l}\text { NADH-Ubiquinone/plastoquinone } \\
\text { (complex I) protein }\end{array}$ & 698.9 & 687.9 & 681.9 & 585.8 & 2256.0 & 3.85 & $4.08 \mathrm{E}-09$ \\
\hline AT3G25150 & $\begin{array}{l}\text { Nuclear transport factor } 2 \text { (NTF2) } \\
\text { family protein with RNA binding } \\
\text { (RRM-RBD-RNP motifs) domain }\end{array}$ & 4.0 & 9.7 & 5.4 & 5.2 & 18.7 & 3.58 & 0.003243 \\
\hline AT1G63855 & Putative methyltransferase family & 14.7 & 26.9 & 22.0 & 16.1 & 54.2 & 3.36 & 0.000234 \\
\hline ATMG01330 & ORF107H & 727.5 & 708.6 & 693.5 & 668.0 & 2186.4 & 3.27 & $2.76 \mathrm{E}-08$ \\
\hline AT2G14580 & ATPRB1, PRB1 & 17.5 & 19.5 & 17.3 & 22.6 & 71.7 & 3.17 & $1.7 \mathrm{E}-05$ \\
\hline AT2G25964 & unknown protein & 44.3 & 42.5 & 39.3 & 39.7 & 122.4 & 3.08 & $2.55 \mathrm{E}-07$ \\
\hline AT2G01422 & $\begin{array}{l}\text { chr2:183940-184734 FORWARD } \\
\text { LENGTH=795 }\end{array}$ & 6.1 & 3.9 & 4.5 & 5.1 & 14.9 & 2.92 & 0.006687 \\
\hline AT5G06640 & $\begin{array}{l}\text { Proline-rich extensin-like family } \\
\text { protein }\end{array}$ & 33.7 & 82.7 & 69.1 & 29.1 & 83.9 & 2.88 & 0.000312 \\
\hline AT2G34260 & $\begin{array}{l}\text { transducin family protein / WD- } 40 \\
\text { repeat family protein }\end{array}$ & 4.2 & 4.8 & 4.5 & 2.8 & 7.8 & 2.79 & 0.04556 \\
\hline AT3G27940 & LBD26 & 14.8 & 13.7 & 14.4 & 5.3 & 14.6 & 2.73 & $6.44 \mathrm{E}-07$ \\
\hline AT2G22980 & SCPL13 & 16.7 & 12.1 & 22.4 & 12.0 & 30.8 & 2.56 & 0.035093 \\
\hline AT5G03350 & Legume lectin family protein & 7.1 & 10.5 & 8.8 & 7.9 & 20.1 & 2.55 & 0.000479 \\
\hline AT2G24270 & ALDH11A3 & 32.3 & 31.4 & 34.1 & 23.3 & 58.6 & 2.52 & 0.013991 \\
\hline AT2G30390 & FC2, FC-II, ATFC-II & 8.8 & 22.8 & 17.3 & 10.0 & 24.6 & 2.47 & 0.001622 \\
\hline AT3G07360 & PUB9, ATPUB9 & 18.6 & 29.5 & 21.1 & 19.6 & 47.3 & 2.42 & 0.015274 \\
\hline AT3G53980 & $\begin{array}{l}\text { Bifunctional inhibitor/lipid-transfer } \\
\text { protein/seed storage } 2 \mathrm{~S} \text { albumin } \\
\text { superfamily protein }\end{array}$ & 131.8 & 138.8 & 128.7 & 120.3 & 287.9 & 2.39 & 0.014004 \\
\hline AT5G35190 & $\begin{array}{l}\text { proline-rich extensin-like family } \\
\text { protein }\end{array}$ & 18.3 & 30.6 & 28.3 & 24.2 & 57.5 & 2.37 & 0.001442 \\
\hline AT5G41670 & $\begin{array}{l}\text { 6-phosphogluconate } \\
\text { dehydrogenase family protein }\end{array}$ & 8.2 & 7.4 & 8.4 & 4.8 & 11.3 & 2.36 & 0.038195 \\
\hline AT4G04220 & AtRLP46, RLP46 & 28.5 & 39.3 & 40.1 & 32.0 & 75.5 & 2.36 & $4.35 \mathrm{E}-06$ \\
\hline AT5G57150 & $\begin{array}{l}\text { basic helix-loop-helix (bHLH) DNA- } \\
\text { binding superfamily protein }\end{array}$ & 18.9 & 56.5 & 48.6 & 20.2 & 47.4 & 2.35 & 0.013083 \\
\hline AT5G39150 & $\begin{array}{l}\text { RmlC-like cupins superfamily } \\
\text { protein }\end{array}$ & 4.5 & 4.9 & 5.1 & 4.1 & 9.6 & 2.34 & 0.002357 \\
\hline AT4G15530 & PPDK & 25.0 & 9.3 & 18.3 & 23.0 & 53.2 & 2.31 & 0.018936 \\
\hline AT1G49180 & protein kinase family protein & 4.9 & 5.0 & 4.8 & 4.1 & 9.4 & 2.31 & 0.007396 \\
\hline AT1G01580 & FRO2, FRD1, ATFRO2 & 253.6 & 449.4 & 293.8 & 255.7 & 584.5 & 2.29 & 0.006986 \\
\hline AT1G77320 & MEI1 & 12.6 & 14.2 & 16.7 & 8.9 & 20.2 & 2.28 & 0.026899 \\
\hline AT4G16960 & $\begin{array}{l}\text { Disease resistance protein (TIR-NBS- } \\
\text { LRR class) family }\end{array}$ & 14.4 & 15.6 & 21.9 & 15.7 & 35.6 & 2.27 & 0.000702 \\
\hline AT1G15520 & $\begin{array}{l}\text { PDR12, ATPDR12, ABCG40, } \\
\text { ATABCG40 }\end{array}$ & 13.8 & 19.1 & 20.5 & 15.0 & 33.2 & 2.21 & 0.000474 \\
\hline AT1G19960 & $\begin{array}{l}\text { BEST Arabidopsis thaliana protein } \\
\text { match is: transmembrane receptors } \\
\text { (TAIR:AT2G32140.1) }\end{array}$ & 48.4 & 88.4 & 67.0 & 47.6 & 105.0 & 2.20 & 0.000246 \\
\hline AT2G22240 & ATMIPS2, MIPS2, ATIPS2 & 32.0 & 44.3 & 40.7 & 22.2 & 49.0 & 2.20 & $5.95 \mathrm{E}-05$ \\
\hline AT3G60330 & AHA7, HA7 & 4.8 & 8.2 & 7.0 & 5.1 & 11.1 & 2.20 & 0.025052 \\
\hline AT4G10040 & CYTC-2 & 66.2 & 77.3 & 61.7 & 53.9 & 118.2 & 2.19 & $6.34 \mathrm{E}-06$ \\
\hline AT2G45930 & unknown protein & 29.0 & 31.0 & 26.0 & 23.3 & 50.0 & 2.15 & 0.000626 \\
\hline AT4G04745 & unknown protein & 23.4 & 17.9 & 13.5 & 23.4 & 50.1 & 2.14 & $1.36 \mathrm{E}-05$ \\
\hline AT3G61430 & PIP1A, ATPIP1, PIP1, PIP1 & 3.8 & 3.9 & 4.8 & 4.3 & 9.1 & 2.14 & 0.023774 \\
\hline AT3G54590 & ATHRGP1, HRGP1 & 40.7 & 56.7 & 54.3 & 33.2 & 70.1 & 2.11 & 0.017076 \\
\hline AT1G44800 & $\begin{array}{l}\text { nodulin MtN21 /EamA-like } \\
\text { transporter family protein }\end{array}$ & 68.6 & 117.0 & 102.8 & 70.8 & 147.9 & 2.09 & 0.000329 \\
\hline AT3G21950 & $\begin{array}{l}\text { S-adenosyl-L-methionine- } \\
\text { dependent methyltransferases } \\
\text { superfamily protein }\end{array}$ & 25.5 & 35.3 & 25.1 & 27.9 & 57.8 & 2.08 & 0.000295 \\
\hline AT1G08320 & TGA9, bZIP21 & 20.8 & 30.3 & 45.4 & 18.1 & 37.3 & 2.07 & 0.034388 \\
\hline AT4G16940 & $\begin{array}{l}\text { Disease resistance protein (TIR-NBS- } \\
\text { LRR class) family }\end{array}$ & 7.2 & 6.2 & 5.3 & 6.0 & 12.4 & 2.07 & 0.002385 \\
\hline AT3G58810 & MTPA2, ATMTPA2, MTP3, АTMTP3 & 20.3 & 22.0 & 18.3 & 12.9 & 26.4 & 2.06 & 0.005308 \\
\hline AT3G61740 & SDG14, ATX3 & 4.7 & 3.7 & 5.2 & 4.1 & 8.4 & 2.05 & 0.021703 \\
\hline AT4G29930 & $\begin{array}{l}\text { basic helix-loop-helix (bHLH) DNA- } \\
\text { binding superfamily protein }\end{array}$ & 14.4 & 17.0 & 43.2 & 12.1 & 24.7 & 2.05 & 0.044011 \\
\hline AT3G12900 & $\begin{array}{l}\text { 2-oxoglutarate (2OG) and Fe(II)- } \\
\text { dependent oxygenase superfamily }\end{array}$ & 111.8 & 270.9 & 167.0 & 109.6 & 224.0 & 2.04 & 0.014966 \\
\hline AT1G70730 & PGM2 & 50.8 & 56.4 & 47.3 & 36.5 & 74.6 & 2.04 & 0.029196 \\
\hline AT5G38020 & $\begin{array}{l}\text { S-adenosyl-L-methionine- } \\
\text { dependent methyltransferases } \\
\text { superfamily protein }\end{array}$ & 115.7 & 177.2 & 155.1 & 74.5 & 152.2 & 2.04 & $1.33 \mathrm{E}-05$ \\
\hline AT3G54580 & Proline-rich extensin-like family & 25.2 & 48.8 & 39.2 & 15.5 & 31.7 & 2.04 & 0.000391 \\
\hline AT4G18970 & $\begin{array}{l}\text { GDSL-like Lipase/Acylhydrolase } \\
\text { superfamily protein }\end{array}$ & 17.1 & 16.3 & 17.1 & 17.6 & 35.9 & 2.04 & $2.16 \mathrm{E}-05$ \\
\hline AT4G04955 & ATALN, ALN & 55.4 & 81.5 & 75.5 & 47.6 & 97.0 & 2.04 & $8.78 \mathrm{E}-06$ \\
\hline AT2G24070 & QWRF4 & 6.1 & 13.3 & 7.5 & 6.5 & 13.3 & 2.03 & 0.0004 \\
\hline AT3G51895 & SULTR3 & 174.7 & 96.9 & 124.7 & 148.5 & 299.5 & 2.02 & $1.5 \mathrm{E}-05$ \\
\hline
\end{tabular}




\begin{tabular}{|c|c|c|c|c|c|c|c|c|}
\hline AT3G19330 & $\begin{array}{l}\text { Protein of unknown function } \\
\text { (DUF677) }\end{array}$ & 7.5 & 18.4 & 17.3 & 8.0 & 16.1 & 2.01 & 0.031041 \\
\hline AT2G27550 & ATC & 121.9 & 84.8 & 70.8 & 92.0 & 184.8 & 2.01 & $7.61 \mathrm{E}-05$ \\
\hline AT2G45070 & SEC61 BETA & 22.2 & 31.2 & 23.5 & 15.2 & 30.0 & 1.98 & 0.009518 \\
\hline AT1G12040 & LRX1 & 19.1 & 26.0 & 28.7 & 14.8 & 29.2 & 1.97 & 0.001745 \\
\hline AT5G67400 & RHS19 & 84.3 & 172.2 & 154.8 & 67.6 & 133.1 & 1.97 & 0.00137 \\
\hline AT4G10860 & unknown protein & 5.3 & 4.9 & 6.4 & 6.4 & 12.7 & 1.97 & 0.000185 \\
\hline AT4G00355 & unknown protein & 19.4 & 21.3 & 25.9 & 13.2 & 25.9 & 1.97 & 0.016704 \\
\hline AT1G76620 & $\begin{array}{l}\text { Protein of unknown function, } \\
\text { DUF547 }\end{array}$ & 78.0 & 77.9 & 77.9 & 53.9 & 105.7 & 1.96 & $5.16 \mathrm{E}-05$ \\
\hline AT4G24420 & $\begin{array}{l}\text { RNA-binding (RRM/RBD/RNP } \\
\text { motifs) family protein }\end{array}$ & 6.7 & 6.9 & 5.9 & 11.0 & 21.5 & 1.96 & 0.000146 \\
\hline AT1G78420 & RING/U-box superfamily protein & 47.8 & 65.8 & 54.7 & 42.2 & 82.6 & 1.96 & 0.008156 \\
\hline AT2G28670 & $\begin{array}{l}\text { Disease resistance-responsive } \\
\text { (dirigent-like protein) family protein }\end{array}$ & 121.4 & 151.5 & 133.3 & 90.1 & 176.5 & 1.96 & 0.00063 \\
\hline AT3G32920 & $\begin{array}{l}\text { P-loop containing nucleoside } \\
\text { triphosphate hydrolases superfamily }\end{array}$ & 3.2 & 6.4 & 4.9 & 4.4 & 8.6 & 1.95 & 0.049185 \\
\hline AT2G37460 & $\begin{array}{l}\text { nodulin MtN21 /EamA-like } \\
\text { transporter family protein }\end{array}$ & 19.7 & 21.4 & 23.5 & 18.6 & 36.2 & 1.95 & 0.00275 \\
\hline AT1G12110 & $\begin{array}{l}\text { NRT1.1, CHL1-1, NRT1, B-1, } \\
\text { ATNRT1, CHL1 }\end{array}$ & 197.4 & 261.0 & 189.5 & 212.3 & 413.1 & 1.95 & 0.000152 \\
\hline AT1G56520 & $\begin{array}{l}\text { Disease resistance protein (TIR-NBS- } \\
\text { LRR class) family }\end{array}$ & 12.3 & 10.4 & 14.4 & 7.2 & 14.0 & 1.94 & 0.004039 \\
\hline AT1G64255 & MuDR family transposase & 9.7 & 9.3 & 8.8 & 10.1 & 19.6 & 1.94 & 0.001477 \\
\hline AT2G33855 & unknown protein & 20.2 & 20.7 & 20.2 & 20.6 & 39.8 & 1.94 & 0.000263 \\
\hline AT1G79530 & GAPCP-1 & 551.4 & 667.1 & 627.6 & 347.7 & 671.0 & 1.93 & $8.44 \mathrm{E}-06$ \\
\hline AT2G48080 & $\begin{array}{l}\text { oxidoreductase, 20G-Fe(II) } \\
\text { oxygenase family protein }\end{array}$ & 28.9 & 21.0 & 21.7 & 34.1 & 65.7 & 1.93 & 0.000252 \\
\hline AT4G16220 & $\begin{array}{l}\text { GDSL-like Lipase/Acylhydrolase } \\
\text { superfamily protein }\end{array}$ & 13.6 & 14.2 & 13.6 & 7.6 & 14.6 & 1.93 & $3.43 E-05$ \\
\hline AT1G28395 & unknown protein & 18.7 & 16.4 & 18.4 & 11.9 & 22.8 & 1.93 & 7.63E-05 \\
\hline AT4G20020 & unknown protein & 240.1 & 247.7 & 259.6 & 233.9 & 448.8 & 1.92 & $4.11 \mathrm{E}-05$ \\
\hline AT2G18230 & AtPPa2, PPa2 & 426.5 & 226.5 & 298.0 & 318.6 & 611.2 & 1.92 & $1.9 \mathrm{E}-05$ \\
\hline AT5G66005 & Expressed protein & 9.2 & 9.4 & 9.4 & 13.8 & 26.4 & 1.92 & 0.032545 \\
\hline AT1G70080 & $\begin{array}{l}\text { Terpenoid cyclases/Protein } \\
\text { prenyltransferases superfamily } \\
\text { protein }\end{array}$ & 7.5 & 6.1 & 6.0 & 6.5 & 12.5 & 1.92 & 0.000325 \\
\hline AT4G26795 & other RNA & 26.5 & 16.9 & 23.7 & 21.4 & 40.9 & 1.91 & 0.013759 \\
\hline AT5G64630 & FAS2, NFB01, NFB1, MUB3.9 & 18.4 & 17.1 & 16.5 & 15.3 & 29.3 & 1.91 & 0.019088 \\
\hline AT3G17712 & unknown protein & 4.1 & 3.7 & 3.5 & 2.9 & 5.6 & 1.91 & 0.049072 \\
\hline AT3G14940 & ATPPC3, PPC3 & 186.0 & 174.9 & 140.2 & 255.1 & 486.1 & 1.91 & $1.71 \mathrm{E}-05$ \\
\hline AT5G06180 & $\begin{array}{l}\text { Protein of unknown function } \\
\text { (DUF1022) }\end{array}$ & 17.4 & 11.7 & 24.6 & 12.9 & 24.6 & 1.91 & 0.001878 \\
\hline AT1G69640 & SBH1 & 307.4 & 313.9 & 324.3 & 242.8 & 461.0 & 1.90 & $5.9 \mathrm{E}-06$ \\
\hline AT4G11550 & $\begin{array}{l}\text { Cysteine/Histidine-rich C1 domain } \\
\text { family protein }\end{array}$ & 17.7 & 22.3 & 22.5 & 15.6 & 29.6 & 1.90 & 0.001593 \\
\hline ATMG01100 & ORF105A & 255.8 & 237.8 & 258.4 & 220.2 & 417.7 & 1.90 & 9.17E-06 \\
\hline AT4G19690 & IRT1 & 183.3 & 350.9 & 303.2 & 238.5 & 451.0 & 1.89 & 0.000196 \\
\hline AT2G20750 & ATEXPB1, EXPB1, ATHEXP BETA 1.5 & 85.3 & 88.0 & 77.3 & 54.1 & 102.3 & 1.89 & $1.37 \mathrm{E}-05$ \\
\hline AT1G78820 & $\begin{array}{l}\text { D-mannose binding lectin protein } \\
\text { with Apple-like carbohydrate- } \\
\text { binding domain }\end{array}$ & 36.1 & 41.9 & 38.7 & 37.3 & 70.4 & 1.89 & $2.57 \mathrm{E}-05$ \\
\hline AT5G51080 & RNase $\mathrm{H}$ family protein & 33.2 & 57.5 & 57.4 & 39.3 & 74.0 & 1.88 & 0.000806 \\
\hline AT2G32960 & $\begin{array}{l}\text { Phosphotyrosine protein } \\
\text { phosphatases superfamily protein }\end{array}$ & 40.5 & 51.3 & 49.2 & 33.7 & 63.5 & 1.88 & 0.005447 \\
\hline AT4G09320 & NDPK1 & 387.1 & 372.3 & 410.8 & 156.0 & 293.3 & 1.88 & $3.77 \mathrm{E}-05$ \\
\hline AT1G30420 & ATMRP12, MRP12, ABCC11 & 14.8 & 18.5 & 24.2 & 11.6 & 21.7 & 1.87 & 0.011465 \\
\hline AT2G07749 & $\begin{array}{l}\text { Mitovirus RNA-dependent RNA } \\
\text { polymerase }\end{array}$ & 29.8 & 30.8 & 32.4 & 20.8 & 39.0 & 1.87 & 0.001033 \\
\hline AT4G23670 & $\begin{array}{l}\text { Polyketide cyclase/dehydrase and } \\
\text { lipid transport superfamily protein }\end{array}$ & 146.2 & 151.8 & 147.0 & 109.9 & 205.0 & 1.86 & $2.35 \mathrm{E}-06$ \\
\hline AT2G43100 & IPMI2, ATLEUD1 & 336.1 & 369.1 & 292.3 & 236.3 & 440.0 & 1.86 & $6.94 \mathrm{E}-07$ \\
\hline AT2G20180 & PIL5 & 166.3 & 154.1 & 170.9 & 150.4 & 280.0 & 1.86 & 0.000634 \\
\hline AT3G47640 & PYE & 14.2 & 13.7 & 23.3 & 12.7 & 23.4 & 1.85 & 0.007259 \\
\hline AT5G64200 & ATSC35, SC35, At-SC35 & 7.7 & 15.7 & 9.1 & 8.0 & 14.8 & 1.85 & 0.03354 \\
\hline AT1G16840 & unknown protein & 6.6 & 5.9 & 6.1 & 7.1 & 13.1 & 1.84 & 0.036544 \\
\hline
\end{tabular}

Genes that are down-regulated in roxy19DS plants ( $F C>1.5, p<0.05)$

\begin{tabular}{|c|c|c|c|c|c|c|c|c|}
\hline \multirow{2}{*}{ AGI code } & \multirow{2}{*}{ Description } & \multicolumn{4}{|c|}{ mean of linear expression values } & \multirow[b]{2}{*}{ roxy19DS } & \multirow{2}{*}{$\begin{array}{l}\text { Nossen/ } \\
\text { roxy19DS }\end{array}$} & \multirow[t]{2}{*}{$p$-value } \\
\hline & & Col-0 & CCMC\#8 & CCMC\#12 & Nossen & & & \\
\hline AT4G07825 & unknown protein & 992.5 & 967.2 & 1045.4 & 1275.1 & 15.4 & 82.6 & $2.85 \mathrm{E}-13$ \\
\hline AT4G05631 & unknown protein & 542.6 & 503.4 & 588.9 & 393.7 & 8.8 & 44.9 & $9.74 \mathrm{E}-13$ \\
\hline AT4G12917 & other RNA & 33.0 & 35.6 & 31.5 & 44.5 & 5.3 & 8.4 & $2.09 \mathrm{E}-09$ \\
\hline AT4G16180 & unknown protein & 365.6 & 339.9 & 357.3 & 283.9 & 114.3 & 2.5 & $4.46 \mathrm{E}-08$ \\
\hline AT4G11570 & $\begin{array}{l}\text { Haloacid dehalogenase-like hydrolase } \\
\text { (HAD) superfamily protein }\end{array}$ & 202.1 & 206.7 & 198.2 & 105.6 & 29.3 & 3.6 & $4.63 \mathrm{E}-08$ \\
\hline AT2G27402 & $\begin{array}{l}\text { plastid transcriptionally active } 18 \\
\text { (TAIR:AT2G32180.1) }\end{array}$ & 77.1 & 56.8 & 72.1 & 98.5 & 7.9 & 12.5 & $5.41 \mathrm{E}-08$ \\
\hline AT2G09795 & other RNA & 95.6 & 98.7 & 100.3 & 100.5 & 7.5 & 13.5 & $6.37 \mathrm{E}-08$ \\
\hline AT4G15700 & Thioredoxin superfamily protein & 33.2 & 33.4 & 39.6 & 46.2 & 6.0 & 7.7 & $6.91 \mathrm{E}-08$ \\
\hline AT1G24300 & GYF domain-containing protein & 134.8 & 120.9 & 137.0 & 105.3 & 31.7 & 3.3 & $1.36 \mathrm{E}-07$ \\
\hline
\end{tabular}




\begin{tabular}{|c|c|c|c|c|c|c|c|c|}
\hline AT4G02600 & MLO1, ATMLO1 & 37.4 & 56.9 & 53.5 & 30.2 & 9.1 & 3.3 & 1.77E-07 \\
\hline AT4G02850 & $\begin{array}{l}\text { phenazine biosynthesis PhzC/PhzF } \\
\text { family }\end{array}$ & 34.2 & 43.7 & 33.7 & 30.1 & 10.2 & 3.0 & $5.16 \mathrm{E}-07$ \\
\hline AT2G35980 & YLS9, NHL10, ATNHL10 & 76.0 & 104.6 & 142.9 & 44.2 & 16.3 & 2.7 & $7.35 \mathrm{E}-07$ \\
\hline AT4G03820 & $\begin{array}{l}\text { Protein of unknown function } \\
\text { (DUF3537) }\end{array}$ & 82.8 & 70.7 & 64.2 & 52.0 & 21.2 & 2.5 & $7.54 \mathrm{E}-07$ \\
\hline AT4G08280 & Thioredoxin superfamily protein & 210.1 & 208.8 & 213.1 & 186.7 & 90.2 & 2.1 & 9.29E-07 \\
\hline AT4G04040 & MEE51 & 412.0 & 411.9 & 430.5 & 470.8 & 243.0 & 1.9 & $1.02 \mathrm{E}-06$ \\
\hline AT2G03340 & WRKY3 & 142.0 & 166.9 & 155.2 & 169.3 & 92.4 & 1.8 & $1.11 \mathrm{E}-06$ \\
\hline AT2G17695 & $\begin{array}{l}\text { FUNCTIONS IN: molecular_function } \\
\text { unknown }\end{array}$ & 115.0 & 91.2 & 95.2 & 77.9 & 10.0 & 7.8 & $1.18 \mathrm{E}-06$ \\
\hline AT1G52100 & Mannose-binding lectin superfamily & 45.3 & 58.2 & 50.1 & 55.7 & 25.7 & 2.2 & $1.43 \mathrm{E}-06$ \\
\hline AT1G70550 & $\begin{array}{l}\text { Protein of Unknown Function } \\
\text { (DUF239) }\end{array}$ & 49.4 & 68.5 & 66.5 & 29.1 & 6.3 & 4.6 & $1.49 \mathrm{E}-06$ \\
\hline AT1G24530 & $\begin{array}{l}\text { Transducin/WD40 repeat-like } \\
\text { superfamily }\end{array}$ & 56.3 & 62.1 & 63.4 & 24.5 & 13.3 & 1.8 & $1.92 \mathrm{E}-06$ \\
\hline AT4G24780 & Pectin lyase-like superfamily protein & 156.3 & 198.4 & 168.0 & 153.6 & 27.8 & 5.5 & $2.82 \mathrm{E}-06$ \\
\hline AT1G30820 & CTP synthase family protein & 453.8 & 241.6 & 276.6 & 407.5 & 205.0 & 2.0 & $3.2 \mathrm{E}-06$ \\
\hline AT4G15690 & Thioredoxin superfamily protein & 27.6 & 22.4 & 25.8 & 14.5 & 8.2 & 1.8 & $3.34 \mathrm{E}-06$ \\
\hline AT2G40920 & $\begin{array}{l}\text { F-box and associated interaction } \\
\text { domains-containing protein }\end{array}$ & 157.6 & 170.0 & 149.5 & 70.7 & 12.3 & 5.8 & $3.43 \mathrm{E}-06$ \\
\hline AT4G02405 & $\begin{array}{l}\text { S-adenosyl-L-methionine-dependent } \\
\text { methyltransferases superfamily protein }\end{array}$ & 46.2 & 44.4 & 49.6 & 60.3 & 31.9 & 1.9 & 4.22E-06 \\
\hline AT2G27050 & EIL1, AtEIL1 & 273.2 & 294.1 & 308.0 & 316.4 & 155.6 & 2.0 & $4.52 \mathrm{E}-06$ \\
\hline AT4G07950 & $\begin{array}{l}\text { DNA-directed RNA polymerase, } \\
\text { subunit } \mathrm{M} \text {, archaeal }\end{array}$ & 80.2 & 82.8 & 83.4 & 63.1 & 15.7 & 4.0 & $4.62 \mathrm{E}-06$ \\
\hline AT2G28930 & APK1B, PK1B & 134.8 & 130.4 & 143.8 & 142.1 & 83.2 & 1.7 & $5.19 E-06$ \\
\hline AT1G08930 & ERD6 & 622.9 & 812.3 & 860.6 & 870.6 & 524.4 & 1.7 & $6.18 \mathrm{E}-06$ \\
\hline AT1G19670 & ATCLH1, CORI1, ATHCOR1, CLH1 & 274.8 & 384.7 & 332.3 & 299.0 & 165.7 & 1.8 & $6.49 \mathrm{E}-06$ \\
\hline AT2G01850 & EXGT-A3, XTH27, ATXTH27 & 294.5 & 294.6 & 333.8 & 332.8 & 158.6 & 2.1 & $6.7 \mathrm{E}-06$ \\
\hline AT4G11900 & S-locus lectin protein kinase family & 71.1 & 82.6 & 85.8 & 46.1 & 16.6 & 2.8 & $1.09 \mathrm{E}-05$ \\
\hline AT2G16660 & Major facilitator superfamily protein & 486.4 & 254.2 & 333.9 & 365.8 & 137.8 & 2.7 & $1.2 \mathrm{E}-05$ \\
\hline AT2G16380 & $\begin{array}{l}\text { Sec14p-like phosphatidylinositol } \\
\text { transfer family protein }\end{array}$ & 141.3 & 149.0 & 146.6 & 103.9 & 59.3 & 1.8 & $1.28 \mathrm{E}-05$ \\
\hline AT2G02180 & TOM3 & 232.3 & 251.8 & 261.2 & 313.0 & 188.6 & 1.7 & $1.44 \mathrm{E}-05$ \\
\hline AT2G29580 & $\begin{array}{l}\mathrm{CCCH} \text {-type zinc fingerfamily protein } \\
\text { with RNA-binding domain }\end{array}$ & 128.9 & 119.0 & 136.7 & 118.9 & 34.5 & 3.4 & $1.53 \mathrm{E}-05$ \\
\hline AT4G14930 & $\begin{array}{l}\text { Survival protein SurE-like } \\
\text { phosphatase/nucleotidase }\end{array}$ & 152.0 & 140.8 & 152.1 & 198.0 & 100.3 & 2.0 & $1.68 \mathrm{E}-05$ \\
\hline AT2G29120 & ATGLR2.7, GLR2.7 & 34.4 & 39.9 & 41.6 & 32.7 & 13.0 & 2.5 & $1.85 \mathrm{E}-05$ \\
\hline AT2G29310 & $\begin{array}{l}\text { NAD(P)-binding Rossmann-fold } \\
\text { superfamily protein }\end{array}$ & 107.4 & 129.9 & 138.7 & 85.4 & 12.8 & 6.7 & $1.91 \mathrm{E}-05$ \\
\hline AT3G57520 & AtSIP2, SIP2 & 1355.3 & 821.4 & 970.6 & 1218.0 & 557.3 & 2.2 & $1.96 \mathrm{E}-05$ \\
\hline AT2G40280 & $\begin{array}{l}\text { S-adenosyl-L-methionine-dependent } \\
\text { methyltransferases superfamily protein }\end{array}$ & 135.5 & 138.9 & 134.3 & 153.3 & 98.8 & 1.6 & $2.03 E-05$ \\
\hline AT4G14590 & emb2739 & 59.8 & 54.5 & 55.4 & 52.4 & 22.8 & 2.3 & $2.52 \mathrm{E}-05$ \\
\hline ATMG01210 & ORF101B & 1047.3 & 985.7 & 1018.6 & 1315.6 & 734.9 & 1.8 & $2.69 \mathrm{E}-05$ \\
\hline AT4G38540 & $\begin{array}{l}\text { FAD/NAD(P)-binding oxidoreductase } \\
\text { family protein }\end{array}$ & 207.6 & 161.6 & 198.1 & 211.6 & 106.0 & 2.0 & $2.72 \mathrm{E}-05$ \\
\hline AT2G26980 & CIPK3 & 275.9 & 220.7 & 245.3 & 183.9 & 87.3 & 2.1 & $2.84 \mathrm{E}-05$ \\
\hline AT2G05540 & Glycine-rich protein family & 1571.1 & 1485.9 & 1552.7 & 1137.0 & 428.7 & 2.7 & $3.18 \mathrm{E}-05$ \\
\hline AT2G02390 & ATGSTZ1, GST18, GSTZ1 & 78.8 & 64.6 & 48.9 & 30.8 & 4.6 & 6.6 & $3.36 \mathrm{E}-05$ \\
\hline AT2G25480 & $\begin{array}{l}\text { TPX2 (targeting protein for Xklp2) } \\
\text { protein family }\end{array}$ & 43.2 & 47.7 & 41.8 & 30.5 & 15.3 & 2.0 & 3.79E-05 \\
\hline AT2G19610 & RING/U-box superfamily protein & 5.8 & 5.7 & 5.5 & 9.3 & 5.1 & 1.8 & $3.95 \mathrm{E}-05$ \\
\hline AT2G28930 & APK1B, PK1B & 7.7 & 7.6 & 6.7 & 10.9 & 4.2 & 2.6 & $4.05 \mathrm{E}-05$ \\
\hline AT3G51600 & LTP5 & 162.7 & 145.6 & 158.8 & 190.6 & 122.8 & 1.6 & $4.24 \mathrm{E}-05$ \\
\hline AT2G17695 & $\begin{array}{l}\text { FUNCTIONS IN: molecular function } \\
\text { unknown }\end{array}$ & 217.5 & 173.3 & 178.3 & 204.8 & 49.8 & 4.1 & 4.49E-05 \\
\hline AT2G41640 & Glycosyltransferase family 61 protein & 84.7 & 80.8 & 79.6 & 116.0 & 38.6 & 3.0 & $4.8 \mathrm{E}-05$ \\
\hline AT4G11410 & $\begin{array}{l}\text { NAD(P)-binding Rossmann-fold } \\
\text { superfamily protein }\end{array}$ & 50.4 & 43.5 & 41.6 & 58.7 & 31.5 & 1.9 & 4.99E-05 \\
\hline AT2G15050 & LTP, LTP7 & 2955.3 & 2779.8 & 2746.9 & 2621.4 & 1341.3 & 2.0 & $5.24 \mathrm{E}-05$ \\
\hline AT1G19080 & TTN10 & 7.4 & 6.7 & 6.5 & 9.1 & 5.9 & 1.5 & $5.83 \mathrm{E}-05$ \\
\hline AT2G20360 & $\begin{array}{l}\text { NAD(P)-binding Rossmann-fold } \\
\text { superfamily protein }\end{array}$ & 675.2 & 672.7 & 658.6 & 638.4 & 410.7 & 1.6 & $6.55 \mathrm{E}-05$ \\
\hline AT1G63260 & TET10 & 74.1 & 68.8 & 75.0 & 57.4 & 35.4 & 1.6 & $6.58 \mathrm{E}-05$ \\
\hline AT1G63830 & PLAC8 family protein & 402.6 & 398.1 & 391.9 & 393.7 & 223.9 & 1.8 & $6.65 \mathrm{E}-05$ \\
\hline AT2G13800 & ATSERK5, SERK5, BAK8 & 41.3 & 44.3 & 50.9 & 41.4 & 18.6 & 2.2 & $6.69 \mathrm{E}-05$ \\
\hline AT4G13640 & UNE16 & 119.5 & 115.3 & 119.5 & 79.7 & 37.9 & 2.1 & $6.82 \mathrm{E}-05$ \\
\hline AT2G17700 & ACT-like protein tyrosine kinase family & 181.8 & 184.8 & 183.8 & 114.4 & 66.5 & 1.7 & $6.91 \mathrm{E}-05$ \\
\hline AT2G25100 & $\begin{array}{l}\text { Polynucleotidyl transferase, } \\
\text { ribonuclease H-like superfamily protein }\end{array}$ & 82.3 & 81.8 & 86.4 & 75.9 & 48.7 & 1.6 & $6.95 \mathrm{E}-05$ \\
\hline AT4G14905 & $\begin{array}{l}\text { Galactose oxidase/kelch repeat } \\
\text { superfamily protein }\end{array}$ & 16.1 & 13.9 & 17.9 & 10.7 & 5.3 & 2.0 & $7.06 \mathrm{E}-05$ \\
\hline AT4G17050 & UGLYAH & 303.5 & 296.3 & 285.9 & 222.0 & 139.7 & 1.6 & 7.12E-05 \\
\hline AT4G02590 & UNE12 & 249.7 & 264.6 & 290.6 & 216.6 & 135.6 & 1.6 & $7.31 \mathrm{E}-05$ \\
\hline AT4G26700 & ATFIM1, FIM1 & 253.3 & 259.6 & 263.4 & 199.7 & 125.1 & 1.6 & $7.38 \mathrm{E}-05$ \\
\hline AT2G11270 & citrate synthase-related & 17.8 & 19.8 & 14.7 & 9.9 & 4.6 & 2.1 & $7.57 \mathrm{E}-05$ \\
\hline AT4G03020 & $\begin{array}{l}\text { transducin family protein / WD- } 40 \\
\text { repeat family protein }\end{array}$ & 199.3 & 179.8 & 192.7 & 220.2 & 132.0 & 1.7 & $7.74 \mathrm{E}-05$ \\
\hline AT3G57520 & AtSIP2, SIP2 & 862.5 & 510.3 & 556.1 & 770.4 & 310.0 & 2.5 & $8.15 \mathrm{E}-05$ \\
\hline AT3G30775 & $\begin{array}{l}\text { ERD5, PRODH, AT-POX, ATPOX, ATPDH, } \\
\text { PRO1 }\end{array}$ & 702.6 & 556.5 & 652.6 & 459.5 & 212.0 & 2.2 & $8.38 \mathrm{E}-05$ \\
\hline AT1G69730 & Wall-associated kinase family protein & 37.3 & 46.6 & 43.3 & 32.7 & 14.3 & 2.3 & $9 \mathrm{E}-05$ \\
\hline
\end{tabular}




\begin{tabular}{|c|c|c|c|c|c|c|c|c|}
\hline AT4G00335 & RHB1A & 85.2 & 82.4 & 86.9 & 110.0 & 58.8 & 1.9 & $9.35 \mathrm{E}-05$ \\
\hline AT1G69890 & Protein of unknown function (DUF569) & 102.0 & 130.6 & 132.4 & 91.6 & 42.5 & 2.2 & $9.54 \mathrm{E}-05$ \\
\hline AT4G21680 & NRT1.8 & 82.4 & 69.4 & 64.7 & 79.3 & 38.5 & 2.1 & $9.55 \mathrm{E}-05$ \\
\hline AT2G19800 & MIOX2 & 991.6 & 779.0 & 924.8 & 1382.7 & 536.1 & 2.6 & 0.000103 \\
\hline AT2G29570 & PCNA2, ATPCNA2 & 151.0 & 146.6 & 138.0 & 107.4 & 37.3 & 2.9 & 0.000105 \\
\hline AT2G03500 & $\begin{array}{l}\text { Homeodomain-like superfamily } \\
\text { protein }\end{array}$ & 157.0 & 149.3 & 150.8 & 179.7 & 111.4 & 1.6 & 0.00011 \\
\hline AT4G02590 & UNE12 & 167.2 & 179.5 & 185.9 & 143.5 & 89.0 & 1.6 & 0.000127 \\
\hline AT5G64570 & XYL4, ATBXL4 & 444.3 & 522.4 & 549.4 & 630.2 & 378.9 & 1.7 & 0.000133 \\
\hline AT4G14870 & SECE1 & 87.3 & 80.8 & 82.6 & 89.0 & 56.3 & 1.6 & 0.00014 \\
\hline AT4G22592 & CPuORF27 & 200.1 & 143.5 & 146.7 & 178.8 & 111.6 & 1.6 & 0.000143 \\
\hline AT2G01090 & $\begin{array}{l}\text { Ubiquinol-cytochrome } \mathrm{C} \text { reductase } \\
\text { hinge }\end{array}$ & 13.1 & 13.4 & 14.1 & 11.2 & 4.0 & 2.8 & 0.000144 \\
\hline AT1G65520 & ATECI1, ECI1, ECHIC, PEC11 & 28.3 & 23.8 & 19.3 & 21.4 & 13.4 & 1.6 & 0.000151 \\
\hline AT1G75220 & Major facilitator superfamily protein & 149.4 & 179.0 & 173.7 & 161.3 & 103.9 & 1.6 & 0.000152 \\
\hline AT1G53440 & $\begin{array}{l}\text { Leucine-rich repeat transmembrane } \\
\text { protein kinase }\end{array}$ & 295.4 & 286.7 & 324.8 & 350.8 & 215.6 & 1.6 & 0.000162 \\
\hline AT2G13100 & Major facilitator superfamily protein & 97.8 & 110.5 & 100.2 & 65.7 & 21.0 & 3.1 & 0.000164 \\
\hline AT2G39900 & $\begin{array}{l}\text { GATA type zinc finger transcription } \\
\text { factor family protein }\end{array}$ & 464.9 & 554.4 & 507.7 & 559.3 & 348.7 & 1.6 & 0.000176 \\
\hline AT2G29950 & ELF4-L1 & 19.1 & 17.6 & 16.0 & 14.6 & 8.0 & 1.8 & 0.0002 \\
\hline AT2G26190 & calmodulin-binding family protein & 99.8 & 124.5 & 120.2 & 101.9 & 63.8 & 1.6 & 0.000211 \\
\hline AT2G39570 & ACT domain-containing protein & 416.7 & 374.1 & 407.8 & 437.7 & 263.4 & 1.7 & 0.000217 \\
\hline AT2G15560 & $\begin{array}{l}\text { Putative endonuclease or glycosyl } \\
\text { hydrolase }\end{array}$ & 65.1 & 60.4 & 58.4 & 54.3 & 32.8 & 1.7 & 0.000219 \\
\hline AT2G09795 & other RNA & 40.0 & 48.1 & 47.5 & 40.0 & 9.4 & 4.2 & 0.000221 \\
\hline AT1G69880 & ATH8, TH8 & 17.6 & 11.2 & 10.3 & 8.6 & 5.2 & 1.6 & 0.000237 \\
\hline AT4G34950 & Major facilitator superfamily protein & 1705.7 & 1656.3 & 1720.0 & 1625.0 & 1004.6 & 1.6 & 0.000257 \\
\hline AT1G76860 & Small nuclear ribonucleoprotein family & 55.8 & 62.8 & 61.4 & 68.9 & 36.9 & 1.9 & 0.000261 \\
\hline AT2G39210 & Major facilitator superfamily protein & 83.3 & 83.8 & 78.4 & 94.7 & 62.7 & 1.5 & 0.000264 \\
\hline AT2G17820 & ATHK1, AHK1, HK1 & 128.1 & 123.5 & 124.0 & 157.8 & 102.3 & 1.5 & 0.000298 \\
\hline AT1G56150 & $\begin{array}{l}\text { SAUR-like auxin-responsive protein } \\
\text { family }\end{array}$ & 63.2 & 45.5 & 55.8 & 62.9 & 37.5 & 1.7 & 0.000308 \\
\hline AT1G22570 & Major facilitator superfamily protein & 86.8 & 73.2 & 98.1 & 107.5 & 45.0 & 2.4 & 0.000314 \\
\hline
\end{tabular}


Table S3 List of genes that are reciprocally regulated in ROXY19 gain- and loss-of-function plants.

\begin{tabular}{|c|c|c|c|c|c|c|c|c|c|c|c|}
\hline \multicolumn{12}{|c|}{ Genes that are down-regulated in 35S:HA-ROXY19CCMC\#8 and up-regulated in roxy19DS (FC>1.5, p<0.05) } \\
\hline \multirow{2}{*}{ AGI code } & \multirow{2}{*}{ Description } & \multicolumn{6}{|c|}{ mean of linear expression values } & \multirow{2}{*}{$\begin{array}{c}\text { Col-0/ } \\
\text { cCMC\#8 }\end{array}$} & \multirow{2}{*}{$p$-value } & \multirow{2}{*}{ roxy19DS/Nossen } & \multirow{2}{*}{$p$-value } \\
\hline & & Col-0 & SSMS\#9 & CCMC\#8 & CCMC\#12 & Nossen & roxy19DS & & & & \\
\hline AT4G15530 & $\begin{array}{l}\text { PPDK I } \\
\text { pyruvate } \\
\text { orthophosphate } \\
\text { dikinase } \\
\text { Cytochrome }\end{array}$ & 25.0 & 18.5 & 9.3 & 18.3 & 23.0 & 53.2 & 2.67 & 0.00722 & 2.31 & 0.01893 \\
\hline AT5G36130 & $\begin{array}{l}\text { P450 } \\
\text { superfamily }\end{array}$ & 51.7 & 50.9 & 24.3 & 19.6 & 55.2 & 95.8 & 2.13 & 0.00017 & 1.73 & 0.00501 \\
\hline AT2G21187 & other RNA & 12.3 & 11.8 & 6.3 & 11.8 & 5.6 & 9.6 & 1.96 & 0.00559 & 1.70 & 0.01812 \\
\hline AT2G18230 & AtPPa2, $\mathrm{PPa} 2$ & 426.5 & 362.6 & 226.5 & 298.0 & 318.6 & 611.2 & 1.88 & 0.00253 & 1.92 & $1.9 \mathrm{E}-05$ \\
\hline AT4G33040 & $\begin{array}{l}\text { Thioredoxin } \\
\text { superfamily }\end{array}$ & 9.3 & 7.4 & 5.0 & 7.1 & 8.0 & 12.5 & 1.85 & 0.00028 & 1.57 & 0.01186 \\
\hline AT3G51895 & $\begin{array}{l}\text { SULTR3;1, } \\
\text { AST12 }\end{array}$ & 174.7 & 182.2 & 96.9 & 124.7 & 148.5 & 299.5 & 1.80 & 0.03529 & 2.02 & $1.5 \mathrm{E}-05$ \\
\hline AT2G44300 & $\begin{array}{l}\text { Bifunctional } \\
\text { inhibitor/lipid- } \\
\text { transfer protein }\end{array}$ & 22.7 & 20.2 & 13.3 & 17.2 & 14.2 & 25.8 & 1.70 & 0.00117 & 1.81 & 0.00512 \\
\hline AT4G37980 & $\begin{array}{l}\text { ELI3-1, ELI3, } \\
\text { ATCAD7, CAD7 }\end{array}$ & 60.0 & 46.9 & 37.0 & 52.6 & 61.4 & 91.0 & 1.62 & 0.00278 & 1.48 & 0.00804 \\
\hline AT2G01422 & $\begin{array}{l}\text { unknown } \\
\text { protein }\end{array}$ & 6.1 & 4.3 & 3.9 & 4.5 & 5.1 & 14.9 & 1.58 & 0.00263 & 2.92 & 0.00668 \\
\hline AT3G50740 & UGT72E1 & 226.9 & 236.9 & 144.6 & 123.1 & 123.2 & 199.9 & 1.57 & 0.00023 & 1.62 & 0.00086 \\
\hline AT4G34000 & ABF3, DPBF5 & 112.8 & 94.6 & 73.0 & 90.0 & 55.8 & 88.6 & 1.55 & 0.02235 & 1.59 & 0.00247 \\
\hline AT4G33070 & $\begin{array}{l}\text { Thiamine } \\
\text { pyrophosphate } \\
\text { dependent } \\
\text { pyruvate } \\
\text { decarboxylase } \\
\text { family protein }\end{array}$ & 70.5 & 87.8 & 46.2 & 47.8 & 40.7 & 72.2 & 1.53 & 0.00356 & 1.77 & 0.00825 \\
\hline
\end{tabular}

\begin{tabular}{|c|c|c|c|c|c|c|c|c|c|c|c|}
\hline \multicolumn{12}{|c|}{ Genes that are down-regulated in 35S:HA-ROXY19CCMC\#8 and up-regulated in roxy19DS (FC>1.5, p<0.05) } \\
\hline \multirow{2}{*}{ AGI code } & \multirow{2}{*}{ Description } & \multicolumn{6}{|c|}{ mean of linear expression values } & \multirow{2}{*}{$\begin{array}{c}\text { CCMC\#8 } \\
\text { /Col-0 }\end{array}$} & \multirow{2}{*}{$p$-value } & \multirow{2}{*}{ Nossen/roxy19DS } & \multirow{2}{*}{$p$-value } \\
\hline & & Col-0 & SSMS\#9 & CCMC\#8 & CCMC\#12 & Nossen & roxy19DS & & & & \\
\hline AT4G01680 & MYB55 & 25.0 & 28.8 & 41.6 & 41.3 & 25.4 & 16.7 & 1.66 & 0.00349 & 1.52 & 0.01065 \\
\hline AT4G02600 & MLO1, ATMLO1 & 7.8 & 8.2 & 11.9 & 9.9 & 12.0 & 7.9 & 1.51 & 0.02774 & 1.52 & 1.77E-07 \\
\hline AT2G26530 & AR781 & 777.5 & 789.3 & 1232.2 & 854.1 & 376.7 & 242.0 & 1.58 & 0.00058 & 1.56 & 0.00484 \\
\hline AT1G52400 & BGLU18 & 72.4 & 90.3 & 116.8 & 107.0 & 31.9 & 15.3 & 1.61 & 0.00952 & 2.08 & 0.04185 \\
\hline АT2G15090 & KCS8 & 77.4 & 104.5 & 131.5 & 104.1 & 55.5 & 22.7 & 1.70 & 0.01269 & 2.45 & 0.00198 \\
\hline AT1G70700 & JAZ9, TIFY7 & 89.8 & 107.7 & 138.8 & 132.3 & 74.8 & 40.4 & 1.55 & 0.01359 & 1.85 & 0.00352 \\
\hline AT3G59880 & $\begin{array}{l}\text { unknown } \\
\text { protein }\end{array}$ & 37.4 & 50.4 & 56.9 & 53.5 & 30.2 & 9.1 & 1.52 & 0.00099 & 3.31 & 0.00351 \\
\hline AT2G26530 & AR781 & 45.3 & 42.3 & 58.2 & 50.1 & 55.7 & 25.7 & 1.28 & 0.04452 & 2.17 & 0.00910 \\
\hline AT1G53340 & $\begin{array}{l}\text { Cysteine/Histidi } \\
\text { ne-rich C1 } \\
\text { domain family } \\
\text { Protein of }\end{array}$ & 12.5 & 15.4 & 22.0 & 18.2 & 13.1 & 8.3 & 1.76 & 0.00463 & 1.57 & 0.02192 \\
\hline AT4G37080 & $\begin{array}{l}\text { unknown } \\
\text { function, } \\
\text { DUF547 }\end{array}$ & 31.8 & 24.9 & 59.6 & 28.8 & 42.4 & 27.9 & 1.88 & 0.04496 & 1.52 & 0.02096 \\
\hline AT1G07960 & ATPDIL5-1 & 263.4 & 329.2 & 427.3 & 348.1 & 326.4 & 188.8 & 1.62 & 0.01400 & 1.73 & 0.03577 \\
\hline
\end{tabular}




\section{Acknowledgement}

After an intensive period of learning and writing, it is a pleasure to complete the thesis by writing an acknowledgment. This thesis reflects not only my research work but also support and help from many people in the past four years.

First and foremost, I would like to express my sincerest thanks and gratitude to my doctoral advisor Prof. Dr. Christiane Gatz for the continuous support of my doctoral research and study, for her patience, motivation and immense knowledge. Her guidance helped me in all the time of research and writing of this thesis. I am also thankful to her for carefully reading and commenting on countless revisions of my thesis in order to achieve high standards.

Thanks are also due to members of my thesis committee and examination board Prof. Dr. Ivo Feussner, Prof. Dr. Volker Lipka, PD Dr. Thomas Teichmann, Jr. Prof. Cynthia Gleason and Prof. Dr. Andrea Polle for generously giving their time and expertise to support and improve this work.

It is a great fortune to work in a friendly and cheerful group. I would like to express my gratitude to Ronald Scholz, Anna Hermann, Katharina Dworak and Larissa Kunz for their excellent technical assistance. Especially, I would like to express my gratitude to Dr. Guido Kriete who managed and maintained all laboratory related issues and stuffs so carefully and efficiently and I could always ask for advice and opinions. I am deeply grateful to Dr. Corinna Thurow for her insightful comments and valuable suggestions and for help analyzing the data. I acknowledge the special contribution of PD Dr. Joachim Uhrig who initiated the exciting project to understand the mechanism and his working attitude inspired me to strive for important science task. I am grateful as well for all former and current colleagues - Sina Barghahn, Ronja Hacke, Florian Jung, Dr. Nathannon Leelarasamee, Ning Li (李宁), Dr. Alexander Meier, Dr. Martin Muthreich, Jan Oberdiek, Dr. Frederik Polzin, Dr. Neena Ratnakaran, Dr. Johanna Schmitz, Julia Schröder, Dr. Armin Töller and Dr. Mark Zander - in the Department of Plant Molecular Biology and Physiology.

I am grateful to many friends here in Göttingen over the years. I greatly value and will cherish their friendship, hospitality, knowledge and wisdom. In many ways, I learned much from them. I doubt that I could ever be able to convey my thanks fully, but I owe everyone my warm appreciation.

Finally, I thank my family for always support me spiritually throughout my life. Words cannot express how much I am indebted to my mother for all of the sacrifices she has made on my behalf. 
